\title{
AN ABSTRACT SETTING \\ FOR DIFFERENTIAL RICCATI EQUATIONS \\ IN OPTIMAL CONTROL PROBLEMS \\ FOR HYPERBOLIC/PETROWSKI-TYPE P.D.E.'S \\ WITH BOUNDARY CONTROL \\ AND SLIGHTLY SMOOTHING OBSERVATION
}

\author{
R. TRIGGIANI
}

\begin{abstract}
We study, by the variational method, the Differential Riccati Equation which arises in the theory of quadratic optimal control problems for 'abstract hyperbolic' equations (which encompass hyperbolic and Petrowski-type partial differential equations (P.D.E.) with boundary control). We markedly relax, at the abstract level, the original assumption of smoothing required of the observation operator by the direct method of [D-L-T.1]. This is achieved, by imposing additional higher level regularity requirements on the dynamics, which, however, are always satisfied by the class of hyperbolic and Petrowski-type mixed P.D.E. problems which we seek to cover. To appreciate the additional level of generality, and related technical difficulties associate with it, it suffices to point out that in the present treatment - unlike in [D-L-T.1] - the gain operator $B^{*} P(t)$ is no longer bounded between the state space $Y$ and the control space $U$. The abstract theory is illustrated by its application to a Kirchoff equation with one boundary control. This requires establishing new higher level interior and boundary regularity results.
\end{abstract}

1991 Mathematics Subject Classification. Primary 93C20; Secondary 35L, 49JK.

Key words and phrases. Differential Riccati equations, optimal control, hyperbolic, Petrowski-type partial differential equations.

Paper presented at the Mini-symposium on "Monotonicity Methods in Nonlinear Analysis", Second World Congress of Nonlinear Analysts, Athens, Greece, July 10-17, 1996.

Research partially supported by the National Science Foundation under Grant DMS9504822, and by the Army Research Office under Grant DAAH04-96-1-0059.

Received: December 6, 1996. 


\section{Introduction. Literature}

This paper presents, in its first part (Sections 1 through 4), a general and unifying abstract treatment of the optimal control problem with quadratic cost functional, over a finite (time) horizon, for the abstract differential equation (1.1) below. Here, $A$ is the generator of a s.c. semigroup and $B$ is a (highly) unbounded control operator, satisfying the 'trace regularity' condition $(\mathrm{H} .1)=(1.6)$ below. This condition was introduced in [L-T.2] (see also [L-T.3], [L-T.6], [F-L-T.1]) and has since been shown to be typical of mixed problems for hyperbolic and Petrowski-type partial differential equations (P.D.E.'s), see [L-T.6]. By duality, the (abstract) 'trace' regularity $(\mathrm{H} .1)=(1.6)$ below for the homogeneous problem (1.1) with $u=0$ is converted into an 'interior' regularity result of the non-homogeneous problem $(1.1)$, see $\left(H .1^{*}\right)=(1.7)$. In this paper, focus and emphasis are placed on the Differential Riccati Equation (D.R.E.) associated with the optimal control problem (1.1)-(1.4), whose non-negative, self-adjoint solution $P(t)$ provides the value of the optimal control problem, as well as the pointwise (a.e. in time) synthesis of the optimal pair, as pointed out by Remark 2.1 below. Applications to mixed problems for hyperbolic and Petrowski-type P.D.E.'s are an integral and essential part of the present study. In Section 5, we illustrate the theory established in Sections 1-4, as it applies to Kirchoff equations. Because of space restrictions, additional P.D.E.'s applications (to Euler-Bernoulli equations, to Schrödinger equations) are provided in a companion paper [T.1] and in a forthcoming book [L-T.8, Chapter 10]. More on this will be said below.

Differential Riccati Equations: Direct method [D-L-T.1] with $R$ smoothing. At the abstract level, the present paper is a conceptual successor of [D-L-T.1]: this work assumed only hypothesis (H.1) $=(1.6)$ on the dynamics (1.1), which in P.D.E.'s applications amounts to a basic level regularity result with $L_{2}$-boundary data, via the equivalent version (H.1*) $=(1.7)$. Following the so-called 'direct method'-from the D.R.E. to the optimal control problem, via dynamic programming-[D-L-T.1] established well-posedness (existence and uniqueness) of the corresponding D.R.E. by local contraction plus global a-priori bounds. Well-posedness of the D.R.E. is, in the present context, non-trivial, due to the high degree of unboundedness of the control operator $B$, expressed by $(1.5 \mathrm{a})$ below, which in P.D.E.'s applications models the action from the boundary to the interior. In [D-L-T.1] success of the direct strategy was based, among other technical issues, on a trick of performing a suitable change of (operator) variable, which made application of the contraction argument more amenable. Once well-posedness of the D.R.E. is established, one then recovers the optimal control problem by dynamic programming. A key point is that the theory of [D-L-T.1] requires, however, the hypothesis that the observation operator $R$ in (1.3) be smoothing, in the sense that

$$
R^{*} R e^{A t} B \text { : continuous } U \rightarrow L_{1}(0, T ; Y) .
$$


For instance, if $\mathcal{A}$ is $(-\Delta)$ with homogeneous Dirichlet B.C., then $(0.1)$ amounts to the smoothing requirement that $R^{*} R$ is comparable to $\mathcal{A}^{-\left(\frac{1}{4}+\epsilon\right)} \times$ $\mathcal{A}^{-\left(\frac{1}{4}+\epsilon\right)}, \epsilon>0$ arbitrary. In return, the critical gain operator $B^{*} P(t)$, which occurs in the quadratic term of the D.R.E. (2.6) (as well as in the optimal synthesis of Remark 2.1) is bounded, at each $t$, from the state space $Y$ to the control space $U$.

Differential Riccati Equation: Variational approach with $R$ almost the identity. One goal of the present paper is to markedly weaken the smoothing requirement (0.1) assumed on $R^{*} R$, in fact, from $R^{*} R \sim$ $\mathcal{A}^{-\left(\frac{1}{4}+\epsilon\right)} \times \mathcal{A}^{-\left(\frac{1}{4}+\epsilon\right)}$ to $R^{*} R \sim \mathcal{A}^{-\epsilon} \times \mathcal{A}^{-\epsilon}, \mathcal{A}$ defined above, see hypothesis $($ H.8 $)=(1.23)$ below, $\epsilon>0$ arbitrary; i.e., $\epsilon$-away from the ideal situation with $R=$ Identity where the observation operator is then non-smoothing. To achieve this quantum improvement over [D-L-T.1], we require in this paper additional abstract regularity assumptions on the dynamics (1.1) [in addition to the basic level $($ H.1 $)=(1.6)$, or $\left(\right.$ H.1 $\left.\left.{ }^{*}\right)=(1.7)\right]$, which amount to a higher level regularity: smoother data imply smoother solutions, in specifically required spaces. In this respect, we hasten to add that: all assumed hypotheses on (1.1) are nothing but actual regularity properties displayed by the 'concrete' classes of hyperbolic and Petrowski-type P.D.E.'s which we seek to cover. In the present paper, our approach is variational-from the optimal control problem to the well-posedness of the D.R.E., thus reversing the 'direct method' of [D-L-T.1]. In return for weakening the assumption on the observation operator $R$, we obtain a less regular theory, not unexpectedly. In contrast with [D-L-T.1], under the present weakened smoothing assumption on $R$ such as $($ H.8) $=(1.23)$, it turns out that:

(1) The gain operator $B^{*} P(t)$ is not bounded any longer from the state space $Y$ to the control space $U$, but only densely defined on $Y$; indeed, its domain is constant in $t$ and coincides with an explicitly identified subspace $\left(Y_{\delta}^{-}\right.$below) of the state space $Y$; see $(2.4)$ for the technical statement.

(2) The present variational approach provides (constructively) existence of the D.R.E., indeed with the operator $P(t)$ defined by $(2.3 \mathrm{a})$ [hence expressible directly in terms of the problem data via (2.1a)] being a nonnegative, self-adjoint solution of the D.R.E. (2.6). Nothing is said about uniqueness now (within a class of non-negative, self-adjoint solutions satisfying the regularity property (2.4)): this is not surprising, and is akin to the situation in the abstract parabolic case [L-T.6], [L-T.7], [L-T.8].

Applications to P.D.E. mixed problems. The setting of the present paper - although abstract - is in reality motivated by, and ultimately directed to, numerous classes of mixed problems for hyperbolic and Petrowskitype partial differential equations defined on a bounded domain $\Omega$ of $R^{n}$, with boundary control. Specifically, these include, but are not limited to, the following cases: (i) second-order hyperbolic equations with Dirichlet 
boundary control; (ii) non-symmetric, non-dissipative, first-order hyperbolic systems with boundary control; (iii) (hyperbolic) Kirchoff equations with finite speed of propagation, as well as (iv) (non-hyperbolic) Euler-Bernoulli equations with infinite speed of propagation with one boundary control (such as they arise in linear elasticity in the special cases where $\operatorname{dim} \Omega=1,2$ ); (v) Schrödinger equations with Dirichlet boundary control. In cases (iii) and (iv), various choices among the two associated boundary conditions are possible, each leading to a different function space setting. So far, the abstract setting for the optimal control problem of the present paper has been successfully applied to all classes (i) through (v). The 'concrete' cases (i) and (ii) were studied in isolation in [L-T.3] and [C-L.1] respectively. Indeed, it was their successful treatment that stimulated the need of producing an all-encompassing abstract framework, by lifting and extracting the essential features common to all these (and other) dynamical P.D.E.'s classes, (i) through (v). These have resulted in seven dynamical assumptions, (H.1) through (H.7) below. We emphasize once more: needless to say, all these assumptions have been verified to hold true for the above classes of hyperbolic [(i)-(iii)] and non-hyperbolic, Petrowski-type [(iv), (v)] mixed problems (with various boundary conditions for (iii) and (iv)). We shall report them in book-form in [L-T.8, Chapter 10]. Indeed, in all these cases these abstract assumptions are, in fact, nothing but distinctive interior and boundary (traces) regularity properties. To be sure, their verification is not a trivial or classical matter, and requires P.D.E. energy methods (not functional analysis techniques), which have been brought to bear only very recently on these mixed P.D.E.'s problems, with emphasis on the basic level with $L_{2}$-boundary data. However, as already pointed out above, our present abstract setting requires also higher level regularity results, both interior and boundary, see e.g., assumptions (H.2) and (H.3) below. In the case of Kirchoff, Euler-Bernoulli and Schrödinger equations, such interior and boundary higher-order regularity results were not available in the literature, and it was our task to provide them. In the case of Kirchoff equations, they are given in Section 5 below, particularly the proof of Theorem 5.8.1 in Section 5.13. In the case of the Euler-Bernoulli and Schrödinger equations, we refer to a companion paper [T.1] and [L-T.8, Chapter 10]. In all these latter three classes, the derivation of higher-level trace regularity results (in space) presents (unexpected) additional difficulties (see Remark 5.13.1) over the known cases of second-order hyperbolic equations with Dirichlet control [L-L-T.1], [L-T.3, Section 3]. In the case of first-order, hyperbolic systems, these higher level results were given in [Rau.1] (see also [C-L.1]), after the basic level regularity result in the fundamental paper [K.1]. We expect that the present setting for the optimal control problem will also apply to additional P.D.E.'s mixed problems, such as the system of elasticity, and the Maxwell equation. 


\section{Mathematical Setting and problem statement}

Dynamical model. We consider the abstract differential equation

$$
\dot{y}=A y+B u \quad \text { on, say, }\left[\mathcal{D}\left(A^{*}\right)\right]^{\prime} ; \quad y(s)=y_{0} \in Y,
$$

or its mild version

$$
\begin{gathered}
y\left(t, s ; y_{0}\right)=e^{A(t-s)} y_{0}+\left(L_{s} u\right)(t), \\
\left(L_{s} u\right)(t)=\int_{s}^{t} e^{A(t-\tau)} B u(\tau) d \tau,
\end{gathered}
$$

where $0 \leq s \leq T<\infty$, subject to the abstract hypotheses listed below.

Optimal control problem on the interval $[s, T]$. We introduce the cost functional

$$
J(u, y)=\int_{s}^{T}\left[\|R y(t)\|_{Z}^{2}+\|u(t)\|_{U}^{2}\right] d t,
$$

and the corresponding optimal control problem O.C.P. is then:

Minimize $J(u, y)$ over all $u \in L_{2}(s, T ; U)$, where $y(t)=y\left(t, s ; y_{0}\right)$

is the solution of Eqn. (1.1) with initial condition $y(s)=y_{0}$.

We now list the abstract assumptions of the present paper.

Abstract assumptions. We first group together in (i) below some standing preliminary basic assumptions:

(i) $U, Y$, and $Z$ are Hilbert spaces; $A$ is the generator of an s.c. semigroup $e^{A t}$ on $Y, t \geq 0 ; B$ is a (linear) continuous operator $U \rightarrow\left[\mathcal{D}\left(A^{*}\right)\right]^{\prime}$, equivalently

$$
A^{-1} B \in \mathcal{L}(U ; Y)
$$

[without loss of generality, we take $A^{-1} \in \mathcal{L}(Y)$. For otherwise we replace $A^{-1}$ with the resolvent operator $R\left(\lambda_{0}, A\right), \lambda_{0}$ a point of the resolvent set of $A$. However, $A^{-1}$ will streamline the notation throughout, e.g., in (1.5b) below, where one would otherwise take the graph norm on $\mathcal{D}\left(A^{*}\right)$.] In (1.1), $A^{*}$ is the $Y$-adjoint of $A$, and $\left[\mathcal{D}\left(A^{*}\right)\right]^{\prime}$ is the Hilbert space dual to the space $\mathcal{D}\left(A^{*}\right) \subset Y$ with respect to the $Y$-topology, with norms

$$
\|y\|_{\mathcal{D}\left(A^{*}\right)}=\left\|A^{*} y\right\|_{Y} ; \quad\|y\|_{\left[\mathcal{D}\left(A^{*}\right)\right]^{\prime}}=\left\|A^{-1} y\right\|_{Y} .
$$

Via (1.5a), we let $\left(B^{*} x, u\right)_{U}=(x, B u)_{Y}$ for $u \in U, x \in \mathcal{D}\left(A^{*}\right)$, and then $B^{*} \in \mathcal{L}\left(\mathcal{D}\left(A^{*}\right) ; U\right)$.

(H.1): (abstract trace regularity) the (closable) operator $B^{*} e^{A^{*} t}$ can be extended as a map

$$
\begin{aligned}
& B^{*} e^{A^{*} t}: \text { continuous } Y \rightarrow L_{2}(0, T ; U) \\
& \int_{0}^{T}\left\|B^{*} e^{A^{*} t} x\right\|_{U}^{2} d t \leq c_{T}\|x\|_{Y}^{2}, \quad x \in Y .
\end{aligned}
$$

Consequently, as seen in [L-T.3, Thm. 1.1], [F-L-T.1, Appendix A], it follows equivalently that the operator $L_{s}$ in $(1.2 \mathrm{~b})$ satisfies 
$\left(\mathrm{H} .1^{*}\right)$

$L_{s}: \quad$ continuous $L_{2}(s, T ; U) \rightarrow C([s, T] ; Y)$ with

a norm which may be made independent of $s$, i.e.,

$$
\left\|L_{s} u\right\|_{C([s, T] ; Y)} \leq c_{T}\|u\|_{L_{2}(s, T ; U)} \text { uniformly in } s .
$$

Then, the operator $L_{s}^{*}$, adjoint of $L_{s}$ in the sense that

$$
\left(L_{s} u, f\right)_{L_{2}(s, T ; Y)}=\left(u, L_{s}^{*} f\right)_{L_{2}(s, T ; U)},
$$

and thus given by

$$
\left(L_{s}^{*} f\right)(t)=\int_{t}^{T} B^{*} e^{A^{*}(\tau-t)} f(\tau) d \tau
$$

satisfies

$L_{s}^{*}: \quad$ continuous $L_{1}(s, T ; Y) \rightarrow L_{2}(s, T ; U)$ with

a norm which may be made independent of $s$, i.e.,

$$
\left\|L_{s}^{*} f\right\|_{L_{2}(s, T ; U)} \leq c_{T}\|f\|_{L_{1}(s, T ; Y)} \text { uniformly in } s .
$$

As stated in the introduction, the above assumption (H.1) was the only hypothesis (in addition to (i)) on the dynamics (1.1), or (1.2), required by the treatment of [D-L-T.1]. The following additional hypotheses (H.2) through (H.7) on the dynamics (1.1) [all verified to be true for the hyperbolic/Petrowski class of P.D.E.'s we intend to cover] will allow us to drastically reduce over [D-L-T.1], the assumption on the degree of smoothing of the observation operator, from $R^{*} R \sim \mathcal{A}^{-\left(\frac{1}{4}+\epsilon\right)} \times \mathcal{A}^{-\left(\frac{1}{4}+\epsilon\right)}$ in [D-L-T.1] to $R^{*} R \sim \mathcal{A}^{-\epsilon} \times \mathcal{A}^{-\epsilon}$ in assumption (H.8) $=$ (1.23) below, where, say, $\mathcal{A}$ is the Laplacian with Dirichlet B.C.

Distinctive new hypotheses. Distinctive new hypotheses over [D-LT.1] are as follows: There exist families of Hilbert spaces (which in applications to P.D.E.'s are Sobolev spaces)

$$
\left\{\begin{array}{l}
U_{\theta} ; Y_{\theta}, 0 \leq \theta \leq \frac{1}{2}+\delta, \text { for some } \frac{1}{2}>\delta>0 ; \theta \neq \frac{1}{2} \\
\text { for } Y_{\theta} ; U_{0}=U ; Y_{0}=Y, \delta \text { henceforth } \\
\text { kept fixed, with the property that: } \\
\quad \text { injection } U_{\theta_{2}} \rightarrow U_{\theta_{1}} \text { and } Y_{\theta_{2}} \rightarrow Y_{\theta_{1}} \\
\quad \text { is compact, } 0 \leq \theta_{1}<\theta_{2} \leq \frac{1}{2}+\delta \\
\text { and the interpolating property } \\
\quad\left[Y_{\frac{1}{2}-\delta},\left[Y_{\frac{1}{2}+\delta}\right]^{\prime}\right]_{\theta=\frac{1}{2}-\delta}=Y, \quad \delta>0,
\end{array}\right.
$$

duality of $\left[Y_{\frac{1}{2}+\delta}\right]^{\prime}$ with respect to $Y$, such that, setting

$$
\begin{aligned}
\mathcal{U}^{\theta}[s, T] & \equiv L_{2}\left(s, T ; U_{\theta}\right) \cap H^{\theta}(s, T ; U) \\
\mathcal{Y}^{\theta}[s, T] & \equiv L_{2}\left(s, T ; Y_{\theta}\right) \cap H^{\theta}(s, T ; Y),
\end{aligned}
$$


where $u \in H^{\theta}(s, T ; U)$ means that the fractional time derivative $D_{t}^{\theta} u \in$ $L_{2}(s, T ; U)$, as usual [L-M.1], with norm

$$
\|u\|_{\mathcal{U}^{\theta}[s, T]}^{2} \equiv\|u\|_{L_{2}\left(s, T ; U_{\theta}\right)}^{2}+\|u\|_{H^{\theta}(s, T ; U)}^{2},
$$

and similarly for $\mathcal{Y}^{\theta}[s, T]$, then:

(H.2)

$L_{s}: \quad$ continuous $\mathcal{U}^{\theta}[s, T] \rightarrow \mathcal{Y}^{\theta}[s, T] \cap C\left([s, T] ; Y_{\theta}\right), \quad 0 \leq \theta<\frac{1}{2}$, with a norm which may be made independent of $s$, i.e.,

$$
\left\|L_{s} u\right\|_{\mathcal{Y}^{\theta}[s, T] \cap C\left([s, T] ; Y_{\theta}\right)} \leq c_{T, \theta}\|u\|_{\mathcal{U}^{\theta}[s, T]} \text { uniformly in } s ; 0 \leq \theta<\frac{1}{2} \text {. }
$$

$\left[\right.$ For $\theta=0,\left(\right.$ H.2) $=(1.14)$ specializes to $\left(H .1^{*}\right)=(1.7)$, via (1.10).]

Remark 1.1. In applications to mixed problems for P.D.E.'s [see Section 5 below, as well as [L-T.3], [C-L.1], [T.1]], one first establishes $(H .1)=(1.6)$, hence the regularity $\left(\mathrm{H} .1^{*}\right)=(1.7)$ for $L_{s}$ [case $\left.\theta=0\right]$; next, one establishes a regularity result for $L_{s}$ for $\theta=1$ involving the spaces $U_{1}$ and $Y_{1}$, which, however, requires a compatibility condition. In interpolating the two above cases $\theta=0$ and $\theta=1$ for $\theta<\frac{1}{2}$, the compatibility condition is irrelevant, and one thus obtains $($ H.2) $=(1.14)$.

(H.3)

$L_{s}^{*}: \quad$ continuous $L_{2}\left(s, T ; Y_{\theta}\right) \rightarrow \mathcal{U}^{\theta}[s, T], 0 \leq \theta \leq \frac{1}{2}+\delta, \theta \neq \frac{1}{2} ;$

with a norm which may be made independent of $s$, i.e.,

$$
\left\|L_{s}^{*} f\right\|_{\mathcal{U}^{\theta}[s, T]} \leq C_{T, \theta}\|f\|_{L_{2}\left(s, T ; Y_{\theta}\right)} \text {, uniformly in } s
$$

[for $\theta=0,($ H.3 $)=(1.15)$ is contained in (1.9)]. Henceforth, we shall fix once and for all a number $\delta>0$ arbitrarily small and set for convenience

$$
\begin{array}{ccc}
Y_{\delta}^{-} \equiv Y_{\theta=\frac{1}{2}-\delta} & \supset & Y_{\delta}^{+} \equiv Y_{\theta=\frac{1}{2}+\delta} \\
U_{\delta}^{-} \equiv U_{\theta=\frac{1}{2}-\delta} & \supset & U_{\delta}^{+} \equiv U_{\theta=\frac{1}{2}+\delta} .
\end{array}
$$

[The values $\theta=\frac{1}{2} \pm \delta$ and $\theta=\frac{1}{2}-\frac{\delta}{2}$ will be the only values of $\theta$ where the assumptions $($ H.2) $=(1.14)$ and $($ H.3 $)=(1.15)$ will be used.]

Remark 1.2. In applications to mixed problems for P.D.E.'s, passage from $Y_{\delta}^{-}=Y_{\frac{1}{2}-\delta}$ to $Y_{\delta}^{+}=Y_{\frac{1}{2}+\delta}$ may represent a jump across compatibility conditions. The meaning of the assumption on $R^{*} R$, made in $(\mathrm{H} .8)=(1.23)$ below, may be precisely this: to perform a passage to by-pass compatibility conditions.

(H.4) (complementing (1.6))

$$
B^{*} e^{A^{*} t}: \text { continuous } Y_{\delta}^{+} \rightarrow C([0, T] ; U) ;
$$

$$
\begin{aligned}
e^{A t} & \text { is also a s.c. semigroup on } Y_{\delta}^{-} \text {and } \mathcal{D}(A) \\
& \text { is dense in } Y_{\delta}^{-} \text {in the } Y_{\delta}^{-} \text {-topology; }
\end{aligned}
$$

$$
A: \text { continuous } Y_{\delta}^{-} \rightarrow\left[Y_{\delta}^{+}\right]^{\prime},
$$


where the duality $\left[Y_{\delta}^{+}\right]^{\prime}$ of $Y_{\delta}^{+}$is with respect to the space $Y$.

(H.7) (complementing (1.5a))

$$
B \text { : continuous } U_{\delta}^{-} \rightarrow\left[Y_{\delta}^{+}\right]^{\prime}
$$

[which is automatically implied by

$$
A^{-1} B \text { : continuous } U_{\delta}^{-} \rightarrow Y_{\delta}^{-}
$$

via assumption $($ H.6) $=(1.20)]$;

(H.8) (assumption on smoothing observation)

$$
R \in \mathcal{L}(Y ; Z) \text { and } R^{*} R: \text { continuous } Y_{\delta}^{-} \rightarrow Y_{\delta}^{+},
$$

which then, by duality, implies

$$
R^{*} R: \text { continuous }\left[Y_{\delta}^{+}\right]^{\prime} \rightarrow\left[Y_{\delta}^{-}\right]^{\prime} .
$$

Remark 1.3. As already noted, in the applications [in the subsequent Section 5 as well as in [L-T.3], [C-L.1], and [T.1]] to P.D.E.'s with boundary control, the spaces $Y_{\delta}^{-}$and $Y_{\delta}^{+}$are Sobolev spaces (which may coincide with domains of appropriate fractional powers of the basic differential operator) invariant under the action of the semigroups $e^{A t}$ and $e^{A^{*} t}$. More insight on the impact of the smoothing assumption $R^{*} R \in \mathcal{L}\left(Y_{\delta}^{-} ; Y_{\delta}^{+}\right)$in (1.23) is provided in the orientation below. Needless to say, for the class of boundary control problems for P.D.E.'s for which this setting is intended [hyperbolic dynamics, plate-like equations, see Section 5 below, as well as [L-T.3], [CL.1], and [T.1]], all basic assumptions (H.1) through (H.7) on $A$ and $B$ are nothing but intrinsic dynamical properties.

Preliminary, direct consequences of the assumptions. Some preliminary, direct consequences of the abstract assumptions, to be invoked in the sequel, are listed next.

(C.1) Putting together $($ H.2 $)=(1.14)$ and $($ H.3 $)=(1.15)$ for $\theta=\frac{1}{2}-\frac{\delta}{2}$, we obtain via (1.11), (1.12), (1.16),

$$
\begin{aligned}
L_{s} L_{s}^{*}: & \text { continuous } L_{2}\left(s, T ; Y_{\frac{\delta}{2}}^{-}\right) \rightarrow C\left([s, T] ; Y_{\frac{\delta}{2}}^{-}\right) \cap H^{\frac{1}{2}-\frac{\delta}{2}}(s, T ; Y) \\
& \subset \mathcal{Y}^{\frac{1}{2}-\frac{\delta}{2}}[s, T] \text { with a norm which may be made } \\
& \text { independent of } s, \text { i.e., } \\
\left\|L_{s} L_{s}^{*} f\right\|_{C\left([s, T] ; Y_{\frac{\delta}{2}}^{-}\right)}+\left\|L_{s} L_{s}^{*} f\right\|_{\mathcal{Y}^{\frac{1}{2}-\frac{\delta}{2}[s, T]}} \leq C_{T, \delta}\|f\|_{L_{2}\left(s, T ; Y_{\frac{\delta}{2}}^{-}\right)}, & \text {uniformly in } s .
\end{aligned}
$$

(C.2) By (strongly) differentiating (1.2b) in $t$, we obtain (at least in $\left.\left[\mathcal{D}\left(A^{*}\right)\right]^{\prime}\right)$

$$
\begin{aligned}
\left(\frac{d L_{s} u}{d t}\right)(t) & =A\left[\int_{s}^{t} e^{A(t-\tau)} B u(\tau) d \tau+A^{-1} B u(t)\right] \\
& =A\left(L_{s} u\right)(t)+B u(t) .
\end{aligned}
$$


Of the possible regularity results which may be given on (1.26), we point out the following one, to be invoked below. By recalling $(\mathrm{H} .2)=(1.14)$ on $L_{s}$ for $\theta=\frac{1}{2}-\delta$, and $($ H.6) $=(1.20),($ H.7 $)=(1.21)$ on $A$ and $B$, respectively, we obtain via (1.26b) and (1.11).

$$
\begin{aligned}
& \frac{d L_{s}}{d t}= A L_{s}+B: \text { continuous } \mathcal{U}^{\frac{1}{2}-\delta}[s, T] \rightarrow L_{2}\left(s, T ;\left[Y_{\delta}^{+}\right]^{\prime}\right) \\
& \text { with a norm which may be made independent of } s, \text { i.e., } \\
&\left\|\frac{d L_{s}}{d t} u\right\|_{L_{2}\left(s, T ;\left[Y_{\delta}^{+}\right]^{\prime}\right)} \leq C_{T, \delta}\|u\|_{\mathcal{U}^{\frac{1}{2}-\delta}[s, T]}, \text { uniformly in } s .
\end{aligned}
$$

(C.3) The assumption that $\mathcal{D}(A)$ is dense in $Y_{\delta}^{-}$, made in $(\mathrm{H} .5)=(1.19)$, implies that: Given $x \in Y_{\delta}^{-}$, there exists $x_{n} \in \mathcal{D}(A)$ such that $\left\|x_{n}-x\right\|_{Y_{\delta}^{-}} \rightarrow$ 0 and

$$
\left\|e^{A t} A x_{n}\right\|_{C\left([0, T] ;\left[Y_{\delta}^{+}\right]^{\prime}\right)}=\left\|A e^{A t} x_{n}\right\|_{C\left([0, T] ;\left[Y_{\delta}^{+}\right]^{\prime}\right)} \leq C_{T}\left\|x_{n}\right\|_{Y_{\delta}^{-}},
$$

by recalling $(\mathrm{H} .6)=(1.20)$ for $A$, and that $e^{A t}$ is a s.c. semigroup on $Y_{\delta}^{-}$ by $($ H.5 $)=(1.19)$. Then, by continuous extension, (1.28) yields

$$
e^{A t} A \text { : continuous } Y_{\delta}^{-} \rightarrow C\left([0, T] ;\left[Y_{\delta}^{+}\right]^{\prime}\right)
$$

under assumption (H.5) and (H.6).

(C.4) Assumption (H.4) $=(1.18)$, by duality, is equivalent to

$$
e^{A t} B \text { : continuous } U \rightarrow C\left([0, T] ;\left[Y_{\delta}^{+}\right]^{\prime}\right) .
$$

Indeed, for $u \in U$ and $y \in\left[Y_{\delta}^{+}\right]^{\prime}$ we compute

$$
\left|\left(e^{A t} B u, y\right)_{Y}\right|=\left|\left(u, B^{*} e^{A^{*} t} y\right)_{U}\right| \leq C_{T}\|u\|_{U}\|y\|_{Y_{\delta}^{+}},
$$

and (1.30) follows then from (1.31).

We then see that assumption (1.30), equivalently $(\mathrm{H} .4)=(1.18)$, for $e^{A t} B=e^{A t} A A^{-1} B$ is implied by the property

$$
A^{-1} B \text { : continuous } U \rightarrow Y_{\delta}^{-},
$$

along with (H.5) and (H.6), since these in turn imply (1.29).

\section{Statement of the main Results}

Our starting point is [D-L-T.1], [L-T.3], which applies by virtue of the assumptions (i), (H.1) $=(1.6)$ and $R \in \mathcal{L}(Y ; Z)$. In the present setting, a far richer and complete theory becomes available.

Theorem 2.1. (Regularity of the optimal pair). Assume hypotheses (i), $($ H.1 $)=(1.6) ;($ H.2 $)=(1.14) ;($ H.3 $)=(1.15) ;($ H.5 $)=(1.19) ;($ H.6 $)=$ $(1.20) ;($ H.7 $)=(1.21)$; and $($ H.8) $=(1.23)$ [actually the weaker requirement $R^{*} R: Y_{\frac{1}{2}-\delta} \rightarrow Y_{\frac{1}{2}}$ will suffice]. Then, the unique optimal pair 
$\left\{u^{0}\left(\cdot, s ; y_{0}\right), y^{0}\left(\cdot, s ; y_{0}\right)\right\}$ of the O.C.P. (1.4) for (1.1) [guaranteed by [D-LT.1]], satisfies the following regularity properties: With $y_{0} \in Y_{\delta}^{-}$defined in (1.16), we have

(i)

$$
\begin{aligned}
y^{0}\left(\cdot, s ; y_{0}\right) & =\left[I_{s}+L_{s} L_{s}^{*} R^{*} R\right]^{-1}\left[e^{A(\cdot-s)} y_{0}\right] \\
& \in C\left([s, T] ; Y_{\delta}^{-}=Y_{\frac{1}{2}-\delta}\right) \cap H^{\frac{1}{2}-\delta}(s, T ; Y) \\
& \subset \mathcal{Y}^{\frac{1}{2}-\delta}[s, T] ;
\end{aligned}
$$

(ii) $\quad u^{0}\left(\cdot, s ; y_{0}\right)=-L_{s}^{*} R^{*} R y^{0}\left(\cdot, s ; y_{0}\right)$

$$
\begin{aligned}
& \in L_{2}\left(s, T ; U_{\delta}^{+}=U_{\frac{1}{2}+\delta}\right) \cap H^{\frac{1}{2}+\delta}(s, T ; U) \\
& \equiv \mathcal{U}^{\frac{1}{2}+\delta}[s, T]
\end{aligned}
$$

a fortiori,

$$
u^{0}\left(\cdot, s ; y_{0}\right) \in C([s, T] ; U)
$$

(see Theorem 3.1.2 below).

All the above results are with norms which may be made independent of $s$.

Theorem 2.2. (Regularity of the gain operator $B^{*} P(t)$ ). Assume hypotheses $(\mathrm{i}),(\mathrm{H} .1)=(1.6)$ through $(\mathrm{H} .8)=(1.23)$. Then, the operator $P(t)$ defined by

$$
\begin{aligned}
P(t) x= & \int_{t}^{T} e^{A^{*}(\tau-t)} R^{*} R y^{0}(\tau, t ; x) d \tau \\
& : \text { continuous } Y \rightarrow C([0, T] ; Y)
\end{aligned}
$$

satisfies the following regularity property

$$
B^{*} P(t): \text { continuous } Y_{\delta}^{-} \rightarrow C([0, T] ; U)
$$

(see Theorem 3.2.1 below).

Remark 2.1. The importance of (2.3) and (2.4) is, of course, that [L-T.3]

$$
J^{0}\left(y_{0}\right) \equiv J\left(u^{0}\left(\cdot, s ; y_{0}\right), y^{0}\left(\cdot, s ; y_{0}\right)=\left(P(s) y_{0}, y_{0}\right)_{Y},\right.
$$

and

$$
u^{0}\left(t, s ; y_{0}\right)=-B^{*} P(t) y^{0}\left(t, s ; y_{0}\right) \in L_{2}(s, T ; U), y_{0} \in Y
$$

Theorem 2.3. (D.R.E.). Assume (i), (H.1) = (1.6) through (H.8) = (1.23). Then, the operator $P(t)$ defined by Eqn. (2.3a) satisfies

$$
(P(t) x, A y)_{Y},(P(t) A x, y)_{Y} \in C[0, T], \forall x, y \in Y_{\delta}^{-},
$$

in the sense that the above quantities, originally defined on $\mathcal{D}(A)$, can be extended on $Y_{\delta}^{-}$; and, moreover, the following Differential Riccati Equation 
for all $0 \leq t<T$ :

$$
\begin{cases}\frac{d}{d t}(P(t) x, y)_{Y}= & -(R x, R y)_{Z}-(P(t) x, A y)_{Y}-(P(t) A x, y)_{Y} \\ & +\left(B^{*} P(t) x, B^{*} P(t) y\right)_{U}, \quad \forall x, y \in Y_{\delta}^{-} \\ P(T)=0 ; & \end{cases}
$$

as well as the corresponding Integral Riccati Equation for all $0 \leq t \leq T$ :

$$
\begin{aligned}
(P(t) x, y)_{Y} & \left.=\int_{t}^{T}\left(R e^{A(\tau-t)} x, R e^{A(\tau-t)} y\right)\right)_{Z} d \tau \\
& -\int_{t}^{T}\left(B^{*} P(\tau) e^{A(\tau-t)} x, B^{*} P(\tau) e^{A(\tau-t)} y\right)_{U} d \tau, x, y \in Y_{\delta}^{-}
\end{aligned}
$$

(see Lemma 4.2.1 and Theorem 4.2.2 below).

Orientation. Existence of a unique optimal pair $\left\{u^{0}\left(\cdot, s ; y_{0}\right)\right.$, $y^{0}\left(\cdot, s ; y_{0}\right\}$ and formulas $(2.1 \mathrm{a}),(2.2 \mathrm{a})$ apply to the present situation [LT.3, pp. 890-891], and we seek to go beyond these preliminary results. Now, Eqns. (1.14) for $L_{s}$ and (1.15) for $L_{s}^{*}$ show, by (1.11), (1.12), that - in the present setting, - the operators $L_{s}$ and $L_{s}^{*}$ do not provide any smoothing in the (Sobolev spaces) $Y_{\theta}$ and $U_{\theta}$, i.e., in what in P.D.E.'s applications will be "the space variable." Thus, in order to achieve a complete theory, which in particular includes the derivation of a Differential Riccati Equation, two main problems of similar nature arise: (1) First, in seeking regularity properties for the optimal trajectory $y^{0}\left(\cdot, s ; y_{0}\right)$ with a "regular" initial datum $y_{0} \in Y_{\delta}^{-}$, one needs to perform a critical bounded inversion of the operator $\left[I_{s}+L_{s} L_{s}^{*} R^{*} R\right]$, which describes $y^{0}\left(\cdot, s ; y_{0}\right)$ (see Eqn. (2.1a)) on the smoother space $L_{2}\left(s, T ; Y_{\delta}^{-}\right)$, in fact on its subspace $\mathcal{Y}^{\frac{1}{2}-\delta}[s, T] \equiv L_{2}\left(s, T ; Y_{\delta}^{-}\right) \cap H^{\frac{1}{2}-\delta}(s, T ; Y)$, see $(1.12)$. This bounded inversion would, however, be a serious problem, unless $L_{s} L_{s}^{*} R^{*} R$ could be asserted to be compact on $\mathcal{Y}^{\frac{1}{2}-\delta}[s, T]$. It is to this end that a "minimal" smoothing assumption on $R^{*} R$, such as, e.g., $($ H.8 $)=(1.23)$, is then invoked [but even the weaker requirement $R^{*} R$ : continuous $Y_{\theta=\frac{1}{2}-\delta} \rightarrow Y_{\theta=\frac{1}{2}}$ would do it, of course]. Once the bounded inversion of $\left[I_{s}+L_{s} L_{s}^{*} R^{*} R\right]$ on $\mathcal{Y}^{\frac{1}{2}-\delta}[s, T]$ is performed, then one obtains, along with assumptions $($ H.5) $=$ (1.19), regularity properties of $y^{0}\left(\cdot, s ; y_{0}\right)$; and hence, via $($ H.3 $)=(1.15)$ applied to the optimality condition, see Eqn. (2.2a) regularity properties of $u^{0}\left(\cdot, s ; y_{0}\right)$, for $s$ fixed. Next, however, in order to obtain the regularity property of the gain operator $B^{*} P(t)$ : continuous $Y_{\delta}^{-} \rightarrow C([0, T] ; U)$ (via [Eqn. (2.3) and Remark 2.1]), we see that we need to refine the preceding result by asserting that, in fact, $\left[I_{s}+L_{s} L_{s}^{*} R^{*} R\right]$ is boundedly invertible on $\mathcal{Y}^{\frac{1}{2}-\delta}[s, T]$, uniformly in $s$. The aforementioned regularity of $B^{*} P(t)$ then justifies the well-posedness of the critical quadratic term, which occurs in the Differential Riccati Equation (2.6). All this summarizes the content of Section 3, which provides the proof of the regularity Theorems 2.1 and 2.2. 
(2) Second, in seeking to derive the Differential Riccati Equation (2.6) on $Y_{\delta}^{-}$, one encounters the obstacle of performing the bounded inversion of the operator $\left[I_{s}+L_{s} L_{s}^{*} R^{*} R\right]$, this time, however, on the weaker space $L_{2}\left(s, T ;\left[Y_{\delta}^{+}\right]^{\prime}\right)$; equivalently, by duality, the bounded inversion of $\left[I_{s}+R^{*} R L_{s} L_{s}^{*}\right]$ on the space $L_{2}\left(s, T ; Y_{\delta}^{+}\right)$. This task would, however, be again a serious problem, unless $R^{*} R L_{s} L_{s}^{*}$ could be asserted to be compact on $L_{2}\left(s, T ; Y_{\delta}^{+}\right)$. It is at this level that the smoothing assumption $R^{*} R$ : continuous $Y_{\delta}^{-} \rightarrow Y_{\delta}^{+}$in $(\mathrm{H} .8)=(1.23)$ is used in full force, as the operator $L_{s}$, by assumption $(\mathrm{H} .2)=(1.14)$, has a known regularity property only on $Y_{\theta}, \theta<\frac{1}{2}$. Accordingly, the bounded inversion of $\left[I_{s}+L_{s} L_{s}^{*} R^{*} R\right]$ on $L_{2}\left(s, T ;\left[Y_{\delta}^{+}\right]^{\prime}\right)$ is then performed for each $s$, a result sufficient in the derivation of the D.R.E. (2.6) in Section 4.

\section{Proofs of theorems 2.1 AND 2.2.}

\subsection{Bounded inVERsion of $\left[I_{s}+L_{s} L_{s}^{*} R^{*} R\right]$} ON THE SPACE $\mathcal{Y}^{\frac{1}{2}-\delta}[s, T]$, UNIFORMLY IN $s$.

Proof of TheOREM 2.1

The key preliminary result is the following.

Theorem 3.1.1. Assume (H.1); (H.2), and (H.3) only for $\theta<\frac{1}{2}$; (H.5); (H.6); (H.7); (H.8) [though the weaker requirement $R^{*} R: Y_{\frac{1}{2}-\delta} \rightarrow Y_{\frac{1}{2}}$ will suffice]. Then:

(i) With reference to the spaces in (1.12) for $\theta=\frac{1}{2}-\delta$ and $\theta=\frac{1}{2}-\frac{\delta}{2}$, we have the following estimate

$$
\left\|L_{s} L_{s}^{*} R^{*} R f\right\|_{\mathcal{Y}^{\frac{1}{2}-\frac{\delta}{2}[s, T]}} \leq C_{T, \delta}\|f\|_{\mathcal{Y}^{\frac{1}{2}-\delta}[s, T]}, \quad \text { uniformly in } s .
$$

(ii) For fixed $s$, the operator $L_{s} L_{s}^{*} R^{*} R: \mathcal{Y}^{\frac{1}{2}-\delta}[s, T] \rightarrow$ itself, is compact, and, in fact, $\left\{L_{s} L_{s}^{*} R^{*} R\right\}$ is a family (in $s$ ) of collectively compact operators on $\mathcal{Y}^{\frac{1}{2}-\delta}[0, T]$, once extended by zero on $[0, s$ ) (in the sense of [An.1, p. 3]).

(iii) For $f \in \mathcal{Y}^{\frac{1}{2}-\delta}[0, T]$, indeed, even $f \in L_{2}\left(0, T ; Y_{\delta}^{-}\right)$, the map $s \rightarrow$ $L_{s} L_{s}^{*} R^{*} R f$ is continuous in $\mathcal{Y}^{\frac{1}{2}-\delta}[0, T]$.

(iv) The operator $\left[I_{s}+L_{s} L_{s}^{*} R^{*} R\right]$ is boundedly invertible on $\mathcal{Y}^{\frac{1}{2}-\delta}[s, T]$, indeed uniformly with respect to $s$ :

$$
\left\|\left[I_{s}+L_{s} L_{s}^{*} R^{*} R\right]^{-1}\right\|_{\mathcal{L}\left(\mathcal{Y}^{\frac{1}{2}-\delta}[s, T]\right)} \leq C_{T, \delta}, \quad \text { uniformly in } s .
$$

Proof. (i) The proof of estimate (3.1.1) is a consequence of part of the following diagram, where all continuity maps are uniform in $s$ : 


$$
\mathcal{Y}^{\frac{1}{2}-\delta}[s, T] \underset{\begin{array}{c}
\text { continuous } \\
\text { by }(1.23)
\end{array}}{\stackrel{R^{*} R}{L}} L_{2}\left(s, T ; Y_{\delta}^{+}\right) \stackrel{\text { continuous }}{\stackrel{\text { injection }}{\longrightarrow}} L_{2}\left(s, T ; Y_{\frac{\delta}{2}}^{-}\right)
$$

In the first step, we use $(\mathrm{H} .8)=(1.23)$ for $R^{*} R$ [but $R^{*} R$ : continuous $Y_{\frac{1}{2}-\delta} \rightarrow Y_{\frac{1}{2}}$ would suffice]; followed by [the combination of $(\mathrm{H} .2)=(1.14)$ and $($ H.3 $)=(1.15)$ for $\theta=\frac{1}{2}-\frac{\delta}{2}$ culminating in] the regularity $($ C.1 $)=$ (1.25); followed in the last step by the

$$
\text { compact injection } \mathcal{Y}^{\frac{1}{2}-\frac{\delta}{2}}[s, T] \rightarrow \mathcal{Y}^{\frac{1}{2}-\delta}[s, T],
$$

a consequence, via (1.12), of the compact injection $Y_{\frac{1}{2}-\frac{\delta}{2}} \rightarrow Y_{\frac{1}{2}-\delta}$ in (1.10) and of $T<\infty$.

(ii) A fortiori from the diagram, $L_{s} L_{s}^{*} R^{*} R$, extended by zero on $[0, s)$ is a compact operator on $\mathcal{Y}^{\frac{1}{2}-\delta}[0, T]$, and the family $\left\{L_{s} L_{s}^{*} R^{*} R\right\}$ is collectively compact (in $s\left[\right.$ A.1, p. 4]) on $\mathcal{Y}^{\frac{1}{2}-\delta}[0, T]$, by estimate (3.1.1). This means that the union, over $0 \leq s \leq s_{0}$, of the image $\left[L_{s} L_{s}^{*} R^{*} R\right]$ (unit ball in $\mathcal{Y}^{\frac{1}{2}-\delta}[0, T]$ ) is a relatively compact set in $\mathcal{Y}^{\frac{1}{2}-\delta}[0, T]$.

(iii) Step 1. Let $g \in \mathcal{U}^{\frac{1}{2}-\delta}[0, T] \equiv L_{2}\left(0, T ; U_{\delta}^{-}\right) \cap H^{\frac{1}{2}-\delta}(0, T ; U)$. We shall first show that, when $L_{s} g$ is extended by zero on $[0, s)$, then

the map $s \rightarrow L_{s} g$ is continuous from $[0, T]$ to $L_{2}\left(0, T ; Y_{\delta}^{-}\right)$.

In fact, with, say, $t>s_{1}>s$, recalling $(1.2 \mathrm{~b})$,

$$
\begin{aligned}
\left\|\left(L_{s} g\right)(t)-\left(L_{s_{1}} g\right)(t)\right\|_{Y_{\delta}^{-}} & =\left\|\int_{s}^{s_{1}} e^{A(t-\tau)} B g(\tau) d \tau\right\|_{Y_{\delta}^{-}} \\
& =\left\|e^{A\left(t-s_{1}\right)} \int_{s}^{s_{1}} e^{A\left(s_{1}-\tau\right)} B g(\tau) d \tau\right\|_{Y_{\delta}^{-}} \\
(\text {by }(1.19)) & \leq C_{T}\left\|\left(L_{s} g\right)\left(s_{1}\right)\right\|_{Y_{\delta}^{-}}
\end{aligned}
$$


where in the last steps we have recalled (1.2b) as well as assumption (H.5) $=(1.19)$ on $e^{A t}$, and $(\mathrm{H} .2)=(1.14)$ with $\theta=\frac{1}{2}-\delta$. Thus, by (3.1.5),

$$
\left\|L_{s} g-L_{s_{1}} g\right\|_{C\left(\left[s_{1}, T\right] ; Y_{\delta}^{-}\right)} \rightarrow 0 \text { as }\left[s_{1}-s\right] \rightarrow 0,
$$

as well as

$$
\left\|L_{s} g-L_{s_{1}} g\right\|_{C\left(\left[s, s_{1}\right] ; Y_{\delta}^{-}\right)}=\left\|L_{s} g\right\|_{C\left(\left[s, s_{1}\right] ; Y_{\delta}^{-}\right)} \rightarrow 0 \text { as }\left[s-s_{1}\right] \rightarrow 0 .
$$

Then, (3.1.6a) and (3.1.6b) a fortiori imply (3.1.4).

Step 2. Next, let $f \in L_{2}\left(0, T ; Y_{\delta}^{-}\right)$. We then show that

the map $s \rightarrow L^{*} f$ is continuous from $[0, T]$ to $\mathcal{U}^{\frac{1}{2}-\delta}[0, T]$.

In fact, the definition (1.8) implies, still with $s_{1}>s$,

$$
\begin{array}{ll}
\|\left(L_{s}^{*} f-L_{s_{1}}^{*} f \|_{\mathcal{U}^{\frac{1}{2}-\delta}[0, T]}\right. & =\left\|L_{s}^{*} f\right\|_{\mathcal{U}^{\frac{1}{2}-\delta}\left[s, s_{1}\right]} \\
(\operatorname{by}(1.15 \mathrm{~b})) & \leq C_{T, \delta}\|f\|_{L_{2}\left(s, s_{1} ; Y_{\delta}^{-}\right)} \rightarrow 0 \text { as }\left[s-s_{1}\right] \rightarrow 0,
\end{array}
$$

after using $(\mathrm{H} .3)=(1.15 \mathrm{~b})$, and $(3.1 .7)$ is proved.

Step 3. Next, with $g=L_{s}^{*} R^{*} R f \in \mathcal{U}^{\frac{1}{2}-\delta}[0, T]$ (conservatively) with $f \in L_{2}\left(0, T ; Y_{\delta}^{-}\right)$, via $(\mathrm{H} .8)=(1.23)$ and $(\mathrm{H} .3)=(1.15)$, we recall $(1.26 \mathrm{~b})$ and write

$$
\begin{aligned}
\frac{d\left(L_{s} g\right)}{d t}=\frac{d\left(L_{s} L_{s}^{*} R^{*} R f\right)}{d t} & =A L_{s} L_{s}^{*} R^{*} R f+B L_{s}^{*} R^{*} R f \\
& =A L_{s} L^{*} R^{*} R f+B L_{s}^{*} R^{*} R f,
\end{aligned}
$$

since, by the definitions $(1.2 \mathrm{~b})$ and $(1.8)$, we have readily $L_{s} L_{s}^{*}=L_{s} L^{*}$. With reference to $(3.1 .10)$, and with $f \in L_{2}\left(0, T ; Y_{\delta}^{-}\right)$, we then have that

the map $s \rightarrow A L_{s} L^{*} R^{*} R f$ continuous in $L_{2}\left(0, T ;\left[Y_{\delta}^{+}\right]^{\prime}\right)$,

by combining (3.1.4) with $g=L^{*} R^{*} R f$ and $($ H.6) $=(1.20)$ on $A$. Also, again with $f \in L_{2}\left(0, T ; Y_{\delta}^{-}\right)$, hence $R^{*} R f \in L_{2}\left(0, T ; Y_{\delta}^{-}\right)$a fortiori

$$
\text { the map } s \rightarrow B L_{s}^{*} R^{*} R f \text { continuous in } L_{2}\left(0, T ;\left[Y_{\delta}^{+}\right]^{\prime}\right) \text {, }
$$

by combining $(3.1 .7)$ and $(\mathrm{H} .7)=(1.21)$ on $B$. Using (3.1.11) and (3.1.12) in (3.1.10) we conclude that:

If $f \in L_{2}\left(0, T ; Y_{\delta}^{-}\right)$, then:

the map $s \rightarrow \frac{d}{d t}\left(L_{s} L_{s}^{*} R^{*} R f\right)$ continuous in $L_{2}\left(0, T ;\left[Y_{\delta}^{+}\right]^{\prime}\right)$,

as well as

$$
\text { the map } s \rightarrow L_{s} L_{s}^{*} R^{*} R f \text { continuous in } L_{2}\left(0, T ; Y_{\delta}^{-}\right) \text {, }
$$
by (3.1.4). 
Step 4. Hence, by interpolation between (3.1.13) and (3.1.14), we obtain, recalling the interpolation property in (1.10),

$$
\begin{aligned}
& \text { the map } s \rightarrow\left(D_{t}^{\theta=\frac{1}{2}-\delta}\right) L_{s} L_{s}^{*} R^{*} R f \text { is continuous in } \\
& \qquad L_{2}\left(0, T ;\left[Y_{\delta}^{-},\left[Y_{\delta}^{+}\right]^{\prime}\right]_{\theta=\frac{1}{2}-\delta}\right)=L_{2}(0, T ; Y),
\end{aligned}
$$

via [L-M, pp. 15, 23]. Then (3.1.14) and (3.1.5) together mean:

If $f \in L_{2}\left(0, T ; Y_{\delta}^{-}\right)$, then:

$$
\text { the map } s \rightarrow L_{s} L_{s}^{*} R^{*} R f \text { continuous in } \mathcal{Y}^{\frac{1}{2}-\delta}[0, T] \text {, }
$$

which proves the desired part (iii).

(iv) We first show that $\left[I_{s}+L_{s} L_{s}^{*} R^{*} R\right]$ is boundedly invertible on the set $\mathcal{Y}^{\frac{1}{2}-\delta}[s, T]$ for each $s$ fixed

$$
\left[I_{s}+L_{s} L_{s}^{*} R^{*} R\right]^{-1} \in \mathcal{L}\left(\mathcal{Y}^{\frac{1}{2}-\delta}[s, T]\right) .
$$

Indeed, since $L_{s} L_{s}^{*} R^{*} R$ is a compact operator on $\mathcal{Y}^{\frac{1}{2}-\delta}[s, T]$ by part (ii), then a (necessary and) sufficient condition for (3.1.17) to hold true is that $\lambda=1$ be not an eigenvalue of $L_{s} L_{s}^{*} R^{*} R$ on $\mathcal{Y}^{\frac{1}{2}-\delta}[s, T]$, which is certainly the case, for otherwise $\lambda=1$ would also be an eigenvalue of $L_{s} L_{s}^{*} R^{*} R$ on $L_{2}(s, T ; Y)$, thus contradicting [L-T.3, p. 891], which asserts that $\left[I_{s}+L_{s} L_{s}^{*} R^{*} R\right]^{-1} \in$ $\mathcal{L}\left(L_{2}(s, T ; Y)\right)$. Thus, $(3.1 .7)$ is proved.

Finally, to assert the uniform estimate (3.1.2), we simply invoke [L-T.3, Lemma 3.12] with $Z_{1} \equiv \mathcal{Y}^{\frac{1}{2}-\frac{\delta}{2}}[0, T]$ with compact injection into $Z_{0} \equiv$ $\mathcal{Y}^{\frac{1}{2}-\delta}[0, T]$, see (3.1.3): this is legal by virtue also of (3.1.1) of part (i), (3.1.16) of part (iii), and (3.1.17) of part (iv). Theorem 3.1.1 is proved.

Remark 3.1.1. In the preceding diagram the weaker requirement $R^{*} R$ : continuous $Y_{\frac{1}{2}-\delta} \rightarrow Y_{\frac{1}{2}}$ would suffice.

Remark 3.1.2. With reference to (2.1a), setting

$$
\Gamma_{s}=\left[I_{s}+L_{s} L_{s}^{*} R^{*} R\right],
$$

we obtain

$$
\Gamma_{s}^{-1}-\Gamma_{s_{1}}^{-1}=\Gamma_{s}^{-1}\left[\Gamma_{s_{1}}-\Gamma_{s}\right] \Gamma_{s_{1}}^{-1}
$$

by the second resolvent equation. Hence, estimate (3.1.2) of Theorem 3.1.1 applied to (3.1.18) readily implies that, for each $f \in \mathcal{Y}^{\frac{1}{2}-\delta}[s, T]$ fixed,

$$
\text { the map } s \rightarrow \Gamma_{s}^{-1} f \text { is continuous in } \mathcal{Y}^{\frac{1}{2}-\delta}[0, T]
$$

a result which can be applied to $y^{0}\left(\cdot, s ; y_{0}\right)$ via $(2.1 \mathrm{a})$. See also Remark 3.1.3 below.

As a corollary of Theorem 3.1.1, we shall prove Theorem 2.1 on the regularity of the optimal pair. 
Theorem 3.1.2. Assume the hypotheses of Theorem 3.1.1: (H.1) through (H.3); (H.5) through (H.8). Then, the optimal pair

$$
\left\{u^{0}\left(\cdot, s ; y_{0}\right), y^{0}\left(\cdot, s ; y_{0}\right)\right\}
$$

guaranteed by [L-T.3], satisfies the following regularity properties for $y_{0} \in$ $Y_{\delta}^{-}$:

$$
\begin{aligned}
\text { (i) } y^{0}\left(\cdot, s ; y_{0}\right) & \equiv \Phi(\cdot, s) y_{0} \in C\left([s, T] ; Y_{\delta}^{-}\right) \cap H^{\frac{1}{2}-\delta}(s, T ; Y) \\
& \subset \mathcal{Y}^{\frac{1}{2}-\delta}[s, T],
\end{aligned}
$$

with norms which may be made independent of $s$ :

$$
\begin{array}{r}
\|\Phi(\cdot, s)\|_{\mathcal{L}\left(C\left([s, T] ; Y_{\delta}^{-}\right) ; Y_{\delta}^{-}\right)}+\|\Phi(\cdot, s)\|_{\mathcal{L}\left(\mathcal{Y}^{\frac{1}{2}-\delta}[s, T] ; Y_{\delta}^{-}\right)} \\
\leq C_{T, \delta}, \text { uniformly in } s ;
\end{array}
$$

(ii) still for $y_{0} \in Y_{\delta}^{-}$,

$$
u^{0}\left(\cdot, s ; y_{0}\right) \in \mathcal{U}^{\frac{1}{2}+\delta}[s, T],
$$

with a norm which may be made independent of $s$,

$$
\left\|u^{0}\left(\cdot, s ; y_{0}\right)\right\|_{\mathcal{L}\left(\mathcal{U}^{\frac{1}{2}+\delta}[s, T] ; Y_{\delta}^{-}\right)} \leq C_{T, \delta} \text {, uniformly in } s \text {. }
$$

Proof. Step 1. We recall Eqn. (2.1a) and (3.1.20a),

$$
y^{0}\left(\cdot, s ; y_{0}\right) \equiv \Phi(\cdot, s) y_{0}=\left[I_{s}+L_{s} L_{s}^{*} R^{*} R\right]^{-1}\left[e^{A(\cdot-s)} y_{0}\right] .
$$

With $y_{0} \in Y_{\delta}^{-}$, we apply $($H.5 $)=(1.19)$, which gives that $e^{A(\cdot-s)}$ is a s.c. semigroup on $Y_{\delta}^{-}$, and finally invoke Theorem 3.1.1(iv), Eqn. (3.1.2), to obtain (3.1.20b) for $\mathcal{L}\left(\mathcal{Y}^{\frac{1}{2}-\delta}[s, T] ; Y_{\delta}^{-}\right)$.

Step 2. We now recall the optimality condition

$$
u^{0}\left(\cdot, s ; y_{0}\right)=-L_{s}^{*} R^{*} R y^{0}\left(\cdot, s ; y_{0}\right),
$$

from Eqn. (2.2a), to which we apply the diagram

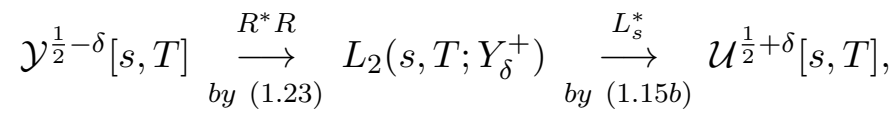

with $y^{0}\left(\cdot, s ; y_{0}\right) \in \mathcal{Y}^{\frac{1}{2}-\delta}[s, T]$ uniformly in $s$ by $(3.1 .20 \mathrm{~b})$ just proved in Step 1. All the maps in the diagram are uniform with respect to $s$, the last one, $L_{s}^{*}$, by $($ H.3 $)=(1.15 \mathrm{~b})$ with $\theta=\frac{1}{2}+\delta$. Then the above diagram and (3.1.23) prove part (ii), i.e., (3.1.21).

Step 3. It remains to complete the proof of part (i), by showing the statement for $C\left([s, T] ; Y_{\delta}^{-}\right)$. To this end, we use the optimal dynamics

$$
y^{0}\left(\cdot, s ; y_{0}\right)=e^{A(\cdot-s)} y_{0}+L_{s} u^{0}\left(\cdot, s ; y_{0}\right)
$$

with $y_{0} \in Y_{\delta}^{-}$, hence $e^{A(\cdot-s)} y_{0} \in C\left([s, T] ; Y_{\delta}^{-}\right)$by (H.5) $=(1.19)$, and finally $L_{s} u^{0}\left(\cdot, s ; y_{0}\right) \in C\left([s, T] ; Y_{\delta}^{-}\right)$by $(\mathrm{H} .2)=(1.14 \mathrm{a})$ with $\theta=\frac{1}{2}-\delta$, since a fortiori from part (ii), $u^{0}\left(\cdot, s ; y_{0}\right) \in \mathcal{U}^{\frac{1}{2}-\delta}[s, T]$. Then, $y^{0}\left(\cdot, s ; y_{0}\right) \in$ 
$C\left([s, T] ; Y_{\delta}^{-}\right)$by (3.1.25). Moreover, all results are uniform in $s$. Theorem 3.1 .2 is fully proved.

Remark 3.1.3. As we have seen, e.g., in the proof of [L-T.3, Lemma 2.1], continuity of $\Phi(t, s) x$ in the first variable, as established by (3.1.20a)

$$
t \rightarrow \Phi(t, s) x \text { continuous in } Y_{\delta}^{-}, \text {for } x \in Y_{\delta}^{-}, \quad T \geq t \geq s,
$$

for $s$ fixed, combined with the uniform bound obtained in $(3.2 .20 \mathrm{~b})$

$$
\|\Phi(t, s)\|_{\mathcal{L}\left(Y_{\delta}^{-}\right)} \leq C_{T} \text { uniformly in } s \leq t \leq T
$$

implies continuity of $\Phi(t, s) x$ in the second variable

$$
s \rightarrow \Phi(t, s) x \text { continuous in } Y_{\delta}^{-}, \text {for } x \in Y_{\delta}^{-}, \quad s \leq t .
$$

\subsection{Proof of TheOREM 2.2}

We restate Theorem 2.2 as

Theorem 3.2.1. Assume hypotheses $($ H.1 $)=(1.6)$ through $($ H.8) $=$ (1.23). Then, the operator $P(t)$ defined by Eqn. (2.3) satisfies

$$
\begin{aligned}
& B^{*} P(t): \text { continuous } Y_{\delta}^{-} \rightarrow C([0, T] ; U) ; \\
& \max _{0 \leq t \leq T}\left\|B^{*} P(t) x\right\|_{U} \leq C_{T}\|x\|_{Y_{\delta}^{-}} .
\end{aligned}
$$

Remark 3.2.1. The weaker statement

$$
B^{*} P(t) \text { : continuous } Y_{\delta}^{-} \rightarrow L_{\infty}(0, T ; U)
$$

can be immediately proved, by applying $($ H.4) $=(1.18),($ H.8) $=(1.23)$ and (3.1.20) of Theorem 3.1.2 (or $(3.1 .27)$ ), to

$$
B^{*} P(t) x=\int_{t}^{T} B^{*} e^{A^{*}(\tau-t)} R^{*} R \Phi(\tau, t) x d \tau .
$$

We obtain with $x \in Y_{\delta}^{-}$:

$$
\left\|B^{*} P(t) x\right\|_{U} \leq C_{T} \int_{t}^{T}\|\Phi(\tau, t) x\|_{Y_{\delta}^{-}} d \tau \leq C_{T, \delta}^{\prime}\|x\|_{Y_{\delta}^{-}},
$$

and (3.2.2) is proved.

Proof of Theorem 3.2.1. Let $t_{1} \in[0, T)$ and let $t>t_{1}$. From (3.2.3), we compute after a change of variable, with $x \in Y_{\delta}^{-}$:

$$
\begin{aligned}
B^{*} P(t) x-B^{*} P\left(t_{1}\right) x= & B^{*} \int_{0}^{T-t} e^{A^{*} \sigma} R^{*} R \Phi(t+\sigma, t) x d \sigma \\
& -B^{*} \int_{0}^{T-t_{1}} e^{A^{*} \sigma} R^{*} R \Phi\left(t_{1}+\sigma, t_{1}\right) x d \sigma \\
= & I_{1}(t) x-I_{2}(t) x
\end{aligned}
$$


where, after adding and subtracting,

$$
\begin{aligned}
& I_{1}(t) x=\int_{0}^{T-t} B^{*} e^{A^{*} \sigma} R^{*} R\left[\Phi(t+\sigma, t) x-\Phi\left(t_{1}+\sigma, t_{1}\right) x\right] d \sigma \\
& I_{2}(t) x=\int_{T-t}^{T-t_{1}} B^{*} e^{A^{*} \sigma} R^{*} R \Phi\left(t_{1}+\sigma, t_{1}\right) x d \sigma .
\end{aligned}
$$

As to $I_{2}(t) x$, we apply $($ H.4 $)=(1.18),($ H.8 $)=(1.23)$ and $(3.1 .21 \mathrm{~b})$ of Theorem 3.1.2, or (3.1.27) to obtain

$$
\begin{aligned}
\left\|I_{2}(t) x\right\|_{U} & \leq C_{T} \int_{T-t}^{T-t_{1}}\left\|\Phi\left(t_{1}+\sigma, t_{1}\right) x\right\|_{Y_{\delta}^{-}} d \sigma \\
& \leq C_{T, \delta}^{\prime}\left(t-t_{1}\right)\|x\|_{Y_{\delta}^{-}} \rightarrow 0 \text { as } t \downarrow t_{1} .
\end{aligned}
$$

As to $I_{1}(t) x$, we again apply $(\mathrm{H} .4)=(1.18)$ and $(\mathrm{H} .8)=(1.23)$ to obtain after adding and subtracting, $\Phi\left(t+\sigma, t_{1}\right) x=\Phi(t+\sigma, t) \Phi\left(t, t_{1}\right) x$ [recall [L-T.3, Lemma 2.1]:

$$
\begin{aligned}
\left\|I_{1}(t) x\right\|_{U} \leq & C_{T} \int_{0}^{T-t}\left\|\Phi(t+\sigma, t) x-\Phi\left(t_{1}+\sigma, t_{1}\right) x\right\|_{Y_{\delta}^{-}} d \sigma \\
\leq & C_{T}\left\{\int_{0}^{T-t}\left\|\Phi(t+\sigma, t) x-\Phi(t+\sigma, t) \Phi\left(t, t_{1}\right) x\right\|_{Y_{\delta}^{-}} d \sigma\right. \\
& \left.+\int_{0}^{T-t}\left\|\Phi\left(t+\sigma, t_{1}\right) x-\Phi\left(t_{1}+\sigma, t_{1}\right) x\right\|_{Y_{\delta}^{-}} d \sigma\right\} .
\end{aligned}
$$

As to the first term on the right-hand side of (3.2.9), we compute

$$
\begin{gathered}
\int_{0}^{T-t}\left\|\Phi(t+\sigma, t)\left[x-\Phi\left(t, t_{1}\right) x\right]\right\|_{Y_{\delta}^{-}} d \sigma \\
\leq \int_{0}^{T-t}\|\Phi(t+\sigma, t)\|_{\mathcal{L}_{\left(Y_{\delta}^{-}\right)}}\left\|x-\Phi\left(t, t_{1}\right) x\right\|_{Y_{\delta}^{-}} d \sigma \\
\text { (by }(3.1 .27)) \leq C_{T, \delta} T\left\|x-\Phi\left(t, t_{1}\right) x\right\|_{Y_{\delta}^{-}} \rightarrow 0 \text { as } t \downarrow t_{1}, \quad x \in Y_{\delta}^{-},
\end{gathered}
$$

after recalling the uniform bound (3.1.27), i.e., (3.1.21b), where convergence to zero attains because of the continuity property in (3.1.17), or (3.1.26). As to the second term in (3.2.9), the integrand, with $[t+\sigma]-\left[t_{1}+\sigma\right]=t-t_{1}$, is uniformly continuous and hence arbitrarily small as $t-t_{1}$ is sufficiently small. Thus

$$
\lim _{t \downarrow t_{1}} \int_{0}^{T-t}\left\|\Phi\left(t+\sigma, t_{1}\right) x-\Phi\left(t_{1}+\sigma, t_{1}\right) x\right\|_{Y_{\delta}^{-}} d \sigma=0 .
$$

Using (3.2.10) and (3.2.11) on the right-hand side of (3.2.9), then yields

$$
\lim _{t \downarrow t_{1}} I_{1}(t) x=0, \quad x \in Y_{\delta}^{-},
$$


as desired. Then, (3.2.8) for $I_{2}(t) x$ and (3.2.12) for $I_{1}(t) x$, used in (3.2.5), complete the proof that

$$
\lim _{t \downarrow t_{1}}\left\|B^{*} P(t) x-B^{*} P\left(t_{1}\right) x\right\|_{U}=0, \quad x \in Y_{\delta}^{-} .
$$

A similar argument applies if $t<t_{1}$, and $t \uparrow t_{1}$. We then obtain that

$$
B^{*} P(t) x \in C\left([0, T] ; Y_{\delta}^{-}\right), \quad x \in Y_{\delta}^{-} .
$$

This, along with (3.2.4), shows (3.2.1), as desired.

Remark 3.2.1. Recalling the pointwise relationship

$$
u^{0}\left(t, 0 ; y_{0}\right)=-B^{*} P(t) y^{0}\left(t, 0 ; y_{0}\right), \quad y_{0} \in Y_{\delta}^{-}
$$

from Remark 2.1, and applying to it the continuity

$$
y^{0}\left(t, 0 ; y_{0}\right) \in C\left([0, T] ; Y_{\delta}^{-}\right)
$$

via (3.1.20) of Theorem 3.1.2, as well as (3.2.1) of Theorem 3.2.1, we reobtain that $u^{0}\left(t, 0 ; y_{0}\right) \in C([0, T] ; U)$, a result a-fortiori contained in $(2.2 \mathrm{~b})$, or $(3.1 .21)$; see $(2.2 \mathrm{c})$.

\section{Proof of THEOREM 2.3}

4.1. Bounded INVERsion OF $\left[I_{s}+L_{s} L_{s}^{*} R^{*} R\right]$ ON THE SPACE $L_{2}\left(s, T ;\left[Y_{\delta}^{+}\right]^{\prime}\right)$. CONSEqUENCES ON $\Phi(t, s)$

We begin with the result which will serve our purposes in the sequel.

Theorem 4.1.1. Assume (i), $(\mathrm{H} .1)=(1.6),(\mathrm{H} .2)=(1.14),(\mathrm{H} .3)=$ (1.15), and $(\mathrm{H} .8)=(1.23)$. Then, for $s$ fixed:

(i) the operator $R^{*} R L_{s} L_{s}^{*}$ is compact on $L_{2}\left(s, T ; Y_{\delta}^{+}\right)$.

(ii) The operator $\left[I_{s}+R^{*} R L_{s} L_{s}^{*}\right]$ is boundedly invertible on $L_{2}\left(s, T ; Y_{\delta}^{+}\right)$:

$$
\left[I_{s}+R^{*} R L_{s} L_{s}^{*}\right]^{-1} \in \mathcal{L}\left(L_{2}\left(s, T ; Y_{\delta}^{+}\right)\right)
$$

(iii) The operator $\left[I_{s}+L_{s} L_{s}^{*} R^{*} R\right]$ is boundedly invertible on $L_{2}\left(s, T ;\left[Y_{\delta}^{+}\right]^{\prime}\right)$ :

$$
\left[I_{s}+L_{s} L_{s}^{*} R^{*} R\right]^{-1} \in \mathcal{L}\left(L_{2}\left(s, T ;\left[Y_{\delta}^{+}\right]^{\prime}\right) .\right.
$$

Proof. (i) The proof of part (i) is a consequence of the following diagram 


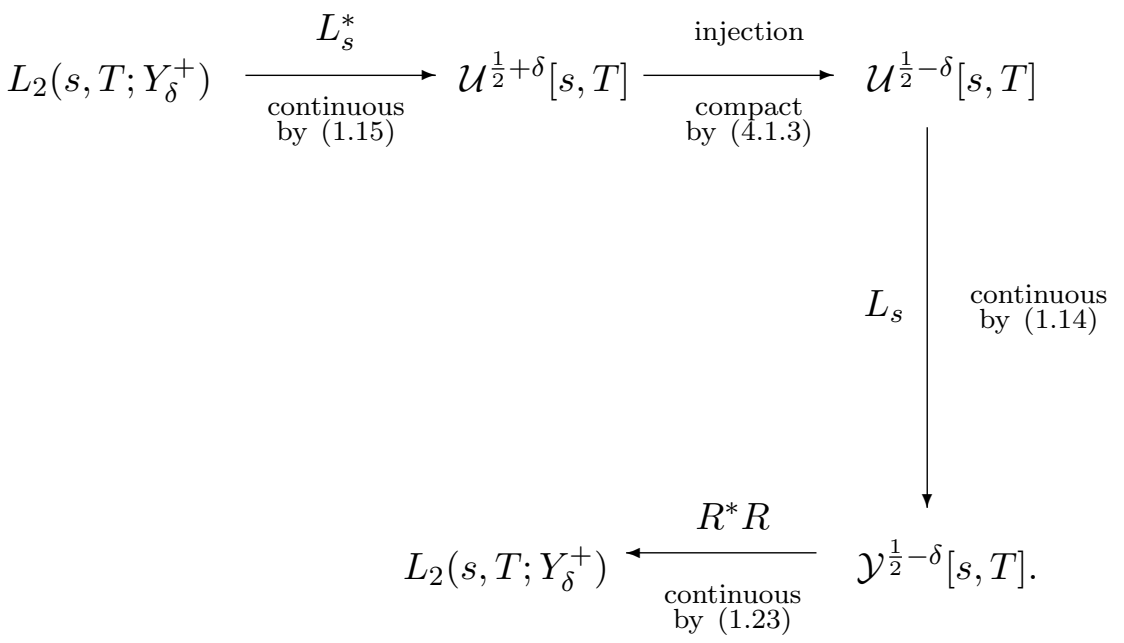

The above diagram uses $($ H.3 $)=(1.15)$ for $\theta=\frac{1}{2}+\delta$ on $L_{s}^{*}$; followed by the

$$
\text { injection } \mathcal{U}^{\frac{1}{2}+\delta}[s, T] \rightarrow \mathcal{U}^{\frac{1}{2}-\delta}[s, T] \text { compact, }
$$

as a consequence of the compactness property $U_{\frac{1}{2}+\delta} \rightarrow U_{\frac{1}{2}-\delta}$ of the injection contained in (1.10) and of $T<\infty$; followed by (H.2) $=(1.14)$ for $\theta=\frac{1}{2}-\delta$ on $L_{s}$; followed by $(\mathrm{H} .8)=(1.23)$ on $R^{*} R$. Thus, as a result, $R^{*} R L_{s} L_{s}^{*}$ is a compact operator on $L_{2}\left(s, T ; Y_{\delta}^{+}\right)$, as desired.

(ii) Since $R^{*} R L_{s} L_{s}^{*}$ is compact on $L_{2}\left(s, T ; Y_{\delta}^{+}\right)$by part (i), then a (necessary and) sufficient condition for (4.1.1) to hold true is that $\lambda=1$ be not an eigenvalue of $R^{*} R L_{s} L_{s}^{*}$ on $L_{2}\left(s, T ; Y_{\delta}^{+}\right)$, which is certainly the case, for otherwise $\lambda=1$ would also be an eigenvalue of $R^{*} R L_{s} L_{s}^{*}$ on $L_{2}(s, T ; Y)$, thus contradicting [L-T.3, p. 891], which asserts that $\left[I_{s}+R^{*} R L_{s} L_{s}^{*}\right]^{-1} \in$ $\mathcal{L}\left(L_{2}(s, T ; Y)\right)$. Thus, (4.1.1) is proved.

(iii) Part (iii), Eqn. (4.1.2), follows from part (ii), Eqn. (4.1.1) by duality.

We can now draw some consequences of Theorem 4.1.1 on properties of the evolution operator $\Phi(t, s)$ in (3.1.20), to be invoked in the sequel

Corollary 4.1.2. Assume preliminarily (i), (H.1) = (1.6) through (H.3) $=(1.15)$ and $($ H.8) $=(1.23)$.

(a) Assume $($ H.5 $)=(1.19)$ on $e^{A t}$, and $($ H.6) $=(1.20)$. Then

$$
\Phi(\cdot, s) A \text { : continuous } Y_{\delta}^{-} \rightarrow L_{2}\left(s, T ;\left[Y_{\delta}^{+}\right]^{\prime}\right),
$$

(ii) Assume (H.4) $=(1.18)$. Then

$$
\Phi(t, s) B \text { : continuous } U \rightarrow L_{2}\left(s, T ;\left[Y_{\delta}^{+}\right]^{\prime}\right) .
$$

(iii) Assume $(\mathrm{H} .4)=($ H.7). Then

$$
\Phi(t, s)\left[A-B B^{*} P(s)\right] \text { : continuous } Y_{\delta}^{-} \rightarrow L_{2}\left(s, T ;\left[Y_{\delta}^{+}\right]^{\prime}\right) \text {. }
$$


Proof. (i) Recalling (3.1.22), we have

$$
\Phi(\cdot, s) A x=\left[I_{s}+L_{s} L_{s}^{*} R^{*} R\right]^{-1}\left[e^{A(\cdot-s)} A x\right],
$$

where, for $x \in Y_{\delta}^{-}$we have $e^{A(\cdot-s)} A x \in C\left([s, T] ;\left[Y_{\delta}^{+}\right]^{\prime}\right)$ by $(\mathrm{C} .3)=(1.29)$, i.e., by (H.5) and (H.6) continuously in $x \in Y_{\delta}^{-}$. Finally, we invoke (4.1.2) of Theorem 4.1.1 and obtain $\Phi(\cdot, s) A x \in L_{2}\left(s, T ;\left[Y_{\delta}^{+}\right]^{\prime}\right)$, continuously in $x \in Y_{\delta}^{-}$, from (4.1.7), as desired.

(ii) Similarly, we have for $u \in U$, via (3.1.22),

$$
\Phi(\cdot, s) B u=\left[I_{s}+L_{s} L_{s}^{*} R^{*} R\right]^{-1}\left[e^{A(\cdot-s)} B u\right],
$$

where now $e^{A(\cdot-s)} B u \in C\left([s, T] ;\left[Y_{\delta}^{+}\right]^{\prime}\right)$ by consequence $(\mathrm{C} .4)=(1.30)$, i.e., duality on $(\mathrm{H} .4)=(1.18)$. Again, (4.1.2) then yields $\Phi(\cdot, s) B u \in$ $L_{2}\left(s, T ;\left[Y_{\delta}^{+}\right]^{\prime}\right)$, continuously in $u \in U$, from (4.1.8), as desired.

(iii) Regularity (4.1.6) is an immediate consequence of (4.1.4) and (4.1.5), via (3.2.1) of Theorem 3.2.1.

Corollary 4.1.3. Assume (H.1) through (H.8). Then,

(i) for $x \in Y_{\delta}^{-}, s \leq t \leq T$,

$$
\frac{d \Phi(t, s) x}{d t}=\left[A-B B^{*} P(t)\right] \Phi(t, s) x \in C\left([s, T] ;\left[Y_{\delta}^{+}\right]^{\prime}\right), \quad x \in Y_{\delta}^{-} ;
$$

(ii) for $x \in Y_{\delta}^{-}, \quad s \leq t \leq T$,

$$
\frac{d \Phi(t, s) x}{d s}=-\Phi(t, s)\left[A-B B^{*} P(t)\right] x \in L_{2}\left(s, T ;\left[Y_{\delta}^{+}\right]^{\prime}\right), \quad x \in Y_{\delta}^{-} \text {. }
$$

Proof. (i) Eqn. (4.1.9) is simply the optimal dynamics in differential form via (3.2.15), and may be obtained by differentiation on its integral version (3.1.25), i.e.,

$$
\Phi(t, s) x=e^{A(t-s)} x-\int_{s}^{t} e^{A(t-\tau)} B B^{*} P(\tau) \Phi(\tau, s) x d \tau,
$$

where the regularity in $C\left([s, T] ;\left[Y_{\delta}^{+}\right]^{\prime}\right)$ in (4.1.9) is obtained by use of assumptions $($ H.6) $=(1.20)$ and $($ H.7 $)=(1.21)$ on $A$ and $B$, respectively, combined with the regularity properties of $\Phi(t, s) x \in C\left([s, T] ; Y_{\delta}^{-}\right)$in $(3.1 .20)$ and (3.2.1) on $B^{*} P(t)$.

(ii) One way to derive (4.1.10) [in line with [L-T.3] is to start from (3.1.22) rewritten as

$$
\Phi(t, s) x+\left\{L_{s} L_{s}^{*} R^{*} R \Phi(\cdot, s) x\right\}(t)=e^{A(t-s)} x, \quad x \in Y_{\delta}^{-},
$$

or, explicitly via (1.2b) and (1.8) as

$$
\begin{aligned}
\Phi(t, s) x & +\int_{s}^{t} e^{A(t-\tau)} B B^{*} \int_{\tau}^{T} e^{A^{*}(\sigma-\tau)} R^{*} R \Phi(\sigma, s) x d \sigma d \tau \\
= & e^{A(t-s)} x
\end{aligned}
$$


take the distributional derivative in $s$, to obtain

$$
\begin{gathered}
{\left[I_{s}+L_{s} L_{s}^{*} R^{*} R\right] \frac{d \Phi(\cdot, s) x}{d s}=-e^{A(\cdot-s)}\left[A-B B^{*} P(s)\right] x} \\
\in C\left([s, T] ;\left[Y_{\delta}^{+}\right]^{\prime}\right) \subset L_{2}\left(s, T ;\left[Y_{\delta}^{+}\right]^{\prime}\right), x \in Y_{\delta}^{-},
\end{gathered}
$$

after invoking the definition of $P(s)$ from Eqn. (2.3a). The regularity displayed at the right-hand side of (4.1.13) is a consequence of (3.2.1) for $B^{*} P(t) ;(\mathrm{C} .3)=(1.28)$ for $e^{A t} A ;(\mathrm{C} .4)=(1.30)$ for $e^{A t} B$. Then, applying (4.1.2) of Theorem 4.1.1 on (4.1.13) yields

$$
\begin{aligned}
\frac{d \Phi(, s) x}{d s} & =-\left[I_{s}+L_{s} L_{s}^{*} R^{*} R\right]^{-1} e^{A(\cdot-s)}\left[A-B B^{*} P(s)\right] x \\
(\operatorname{by}(3.1 .22)) & =-\Phi(\cdot, s)\left[A-B B^{*} P(s)\right] x \\
& \in L_{2}\left(s, T ;\left[Y_{\delta}^{+}\right]^{\prime}\right), \quad x \in Y_{\delta}^{-},
\end{aligned}
$$

and (4.1.10) is proved.

Alternatively, writing

$$
\Phi(t, \tau) x=\Phi(t, s) \Phi(s, \tau) x, \quad \tau \leq s \leq t, x \in Y_{\delta}^{-},
$$

by the evolution property of [L-T.3, Lemma 2.1] or [D-L-T.1], we differentiate both sides of (4.1.15) in $s$, e.g., as a distributional derivative, obtaining

$$
0=\frac{d \Phi(t, \tau) x}{d s}=\frac{d \Phi(t, s)}{d s} \Phi(s, \tau) x+\Phi(t, s) \frac{d \Phi(s, \tau) x}{d s},
$$

or using (4.1.6) and (3.1.26) or (3.1.20a)

$$
\begin{aligned}
\frac{d \Phi(t, s)}{d s} \Phi(s, \tau) x & =-\Phi(t, s)\left[A-B B^{*} P(s)\right] \Phi(s, \tau) x \\
& \in \quad L_{2}\left(s, T ;\left[Y_{\delta}^{+}\right]^{\prime}\right), \quad x \in Y_{\delta}^{-}, \\
& \text {in } t
\end{aligned}
$$

recalling the regularity of (4.1.6) combined with that of $\Phi(t, s)$ on $Y_{\delta}^{-}$given by (3.1.20a). Since (4.1.17) is valid for all $\tau \leq s$, setting $\tau=s$ yields (4.1.10), as desired.

\subsection{Derivation of the Differential \\ AND INTEGRAL RICCATI EQUATIONS}

Lemma 4.2.1. Assume (i), (H.1) $=(1.6)$ through $(\mathrm{H} .3)=(1.15),(\mathrm{H} .5)$ $=(1.19)$ through $(\mathrm{H} .8)=(1.23)$. Then, with reference to the non-negative, self-adjoint operator $P(t) \in \mathcal{L}(Y)$ defined by Eqn. (2.3a), we have

$$
A^{*} P(t) \text { : continuous } Y_{\delta}^{-} \rightarrow C\left([0, T] ;\left[Y_{\delta}^{-}\right]^{\prime}\right),
$$

so that, for $x, y \in Y_{\delta}^{-}$, we have the duality pairings

$$
(P(t) x, A y)_{Y},(P(t) A x, y)_{Y} \in C[0, T]
$$


Proof. (i) We examine

$$
A^{*} P(t) x=\int_{t}^{T} A^{*} e^{A^{*}(\tau-t)} R^{*} R \Phi(\tau, t) x d \tau
$$

for $x \in Y_{\delta}^{-}$. Then, by (3.1.20), or (3.1.26), and by (H.8) $=(1.23)$, we have

$$
R^{*} R \Phi(\tau, t) x \in C\left([t, T] ; Y_{\delta}^{+}\right), \quad x \in Y_{\delta}^{-},
$$

and by duality on $($ C.3) $=(1.29)$, we have

$$
A^{*} e^{A^{*} t}: \text { continuous } Y_{\delta}^{+} \rightarrow C\left([0, T] ;\left[Y_{\delta}^{-}\right]^{\prime}\right) .
$$

Using (4.2.4) and (4.2.5) in (4.2.3) yields $A^{*} P(t) x \in\left[Y_{\delta}^{-}\right]^{\prime}$. Actually, since by $(3.1 .28)$ and $(4.2 .5)$, respectively,

$$
\begin{aligned}
& t \rightarrow \Phi(\tau, t) x \text { continuous in } Y_{\delta}^{-}, \text {for } x \in Y_{\delta}^{-} \\
& t \rightarrow A^{*} e^{A^{*}(\tau-t)} y \text { continuous in }\left[Y_{\delta}^{-}\right]^{\prime} \text { for } y \in Y_{\delta}^{+},
\end{aligned}
$$

then, in fact, $\left.A^{*} P(t) x \in C[0, T] ;\left[Y_{\delta}^{-}\right]^{\prime}\right), \quad x \in Y_{\delta}^{-}$, as desired. The closed graph theorem then yields (4.2.1).

Part (ii), Eqn. (4.2.2), is an immediate consequence of part (i) and of $P(t)$ being self-adjoint on $Y$.

Remark 4.2.1. Notice that we would have:

$$
\begin{aligned}
P(t): & \text { continuous } Y_{\delta}^{-} \rightarrow C\left([0, T] ; Y_{\delta}^{+}\right), \\
& \text {if and only if } A \text { is an isomorphism } Y_{\delta}^{-} \text {onto }\left[Y_{\delta}^{+}\right],
\end{aligned}
$$

a property for $A$ which is generally false; see illustrations below.

We can finally establish that $P(t)$ satisfies the D.R.E.

Theorem 4.2.2. Assume (i), (H.1) through (H.8). Then the nonnegative, self-adjoint operator $P(t)$ defined by (2.3a) satisfies the following Differential Riccati Equation for all $0 \leq t<T$ :

$$
\begin{cases}\frac{d}{d t}(P(t) x, y)_{Y}= & -(R x, R y)_{Z}-(P(t) x, A y)_{Y}-(P(t) A x, y)_{Y} \\ & +\left(B^{*} P(t) x, B^{*} P(t) y\right)_{U}, \quad \forall x, y \in Y_{\delta}^{-} \\ P(T)=0 & \end{cases}
$$

Proof. Let $x, y \in Y_{\delta}^{-}$. We differentiate in $t$ the expression

$$
(P(t) x, y)_{Y}=\int_{t}^{T}\left(R^{*} R \Phi(\tau, t) x, e^{A(\tau-t)} y\right)_{Y} d \tau
$$


obtained from Eqn. (2.3a), thus obtaining

$$
\begin{aligned}
\frac{d}{d t}(P(t) x, y)_{Y}= & -\left(R^{*} R x, y\right)_{Y} \\
& -\int_{t}^{T}\left(R^{*} R \frac{\partial \Phi(\tau, t) x}{\partial t}, e^{A(\tau-t)} y\right)_{Y} d \tau \\
& -\int_{t}^{T}\left(R^{*} R \Phi(\tau, t) x, e^{A(\tau-t)} A y\right)_{Y} d \tau \\
(\text { by }(4.1 .10))= & -\left(R^{*} R x, y\right)_{Y} \\
& -\int_{t}^{T}\left(R^{*} R \Phi(\tau, t)\left[A-B B^{*} P(t)\right] x, e^{A(\tau-t)} y\right)_{Y} d \tau \\
& -(P(t) x, A y)_{Y}, \quad x, y \in Y_{\delta}^{-},
\end{aligned}
$$

after substituting (4.1.10) in the second term on the right-hand side of (4.2.12), as well as substituting (4.2.11) [with $y$ replaced by $A y$ ] in the third term on the right-hand side of (4.2.12). We notice explicitly that each term in (4.2.12), or (4.2.13), is well-defined at each $t$ : the last term by (4.2.2), and the critical second term on the right-hand side of (4.2.12), or (4.2.13), by the regularity in (4.1.10) for $\frac{d \phi(\tau, t) x}{d t}$, combined with $R^{*} R$ : continuous $\left[Y_{\delta}^{+}\right]^{\prime} \rightarrow\left[Y_{\delta}^{-}\right]^{\prime}$ by (1.24), as well as with

$$
e^{A(\tau-t)} y \in C\left([t, T] ; Y_{\delta}^{-}\right) \text {for } y \in Y_{\delta}^{-} \text {, by }(\text { H.5 })=(1.19) \text {. }
$$

Thus, invoking again (4.2.11) on the second term on the right-hand side of (4.2.13), we obtain

$$
\begin{aligned}
\frac{d}{d t}(P(t) x, y)_{Y}= & -\left(R^{*} R x, y\right)_{Y} \\
& -\left(P(t)\left[A-B B^{*} P(t)\right] x, y\right)_{Y}-(P(t) x, A y)_{Y} \\
= & -\left(R^{*} R x, y\right)_{Y}-(P(t) A x, y)_{Y}-(P(t) x, A y)_{Y} \\
& +\left(B^{*} P(t) x, B^{*} P(t) y\right)_{U}, x, y \in Y_{\delta}^{-}
\end{aligned}
$$

each term being well defined, by virtue of (3.2.1) and (4.2.2). Then, (4.2.14) proves (4.2.10), as desired.

As a consequence of Theorem 4.2.2, we obtain that the operator $P(t)$ satisfies the Integral Riccati Equation as well.

Theorem 4.2.3. Assume (i), (H.1) $=(1.6)$ through $(\mathrm{H} .8)=(1.23)$. Then, the non-negative, self-adjoint operator $P(t)$ of Theorem 4.2 .2 satisfies 
the following Integral Riccati Equation for all $0 \leq t<T$.

$$
\begin{aligned}
(P(t) x, y)_{Y} & =\int_{t}^{T}\left(R e^{A(\tau-t)} x, R e^{A(\tau-t)} y\right)_{Z} d \tau \\
& -\int_{t}^{T}\left(B^{*} P(\tau) e^{A(\tau-t)} x, B^{*} P(\tau) e^{A(\tau-t)} y\right)_{U} d \tau, x, y \in Y_{\delta}^{-},
\end{aligned}
$$

where all terms are well defined by (1.19), (3.2.1), (1.23).

Proof. For $x, y \in Y_{\delta}^{-}$, we compute

$$
\begin{aligned}
\frac{d}{d \tau}\left(e^{A^{*}(\tau-t)} P(\tau) e^{A(\tau-t)} x, y\right)_{Y} & \\
= & \frac{d}{d \tau}\left(P(\tau) e^{A(\tau-t)} x, e^{A(\tau-t)} y\right)_{Y} \\
= & \left.\frac{\partial}{\partial r}\left(P(r) e^{A(\tau-t)} x, e^{A(\tau-t)} y\right)_{Y}\right|_{r=\tau} \\
& +\left(P(\tau) e^{A(\tau-t)} A x, e^{A(\tau-t)} y\right)_{Y} \\
& +\left(P(\tau) e^{A(\tau-t)} x, e^{A(\tau-t)} A y\right)_{Y},
\end{aligned}
$$

where by using the D.R.E. (4.2.10), we have

$$
\begin{aligned}
\frac{\partial}{\partial r}( & \left.P(r) e^{A(\tau-t)} x, e^{A(\tau-t)} y\right)\left.\right|_{r=\tau} \\
= & -\left(R^{*} R e^{A(\tau-t)} x, e^{A(\tau-t)} y\right)_{Y}-\left(P(\tau) e^{A(\tau-t)} x, A e^{A(\tau-t)} y\right)_{Y} \\
& -\left(P(\tau) A e^{A(\tau-t)} x, e^{A(\tau-t)} y\right)_{Y} \\
& +\left(B^{*} P(\tau) e^{A(\tau-t)} x, B^{*} P(\tau) e^{A(\tau-t)} y\right)_{U}, x, y \in Y_{\delta}^{-}
\end{aligned}
$$

We note explicitly that each term of (4.2.16) and (4.2.17) is well defined, indeed, we have $e^{A(\tau-t)} x, e^{A(\tau-t)} y$ in $C\left([t, T] ; Y_{\delta}^{-}\right)$, for $x, y \in Y_{\delta}^{-}$, and hence:

$$
\begin{gathered}
P(\tau) e^{A(\tau-t)} x \in C\left([t, T] ; Y_{\delta}^{+}\right), \text {by }(4.2 .3), \\
A e^{A(\tau-t)} x \in C\left([0, T] ;\left[Y_{\delta}^{+}\right]^{\prime}\right), \text { by }(1.19),(1.20), \\
B^{*} P(\tau) e^{A(\tau-t)} x \in C([t, T] ; U), \text { by }(3.2 .1),(1.19),
\end{gathered}
$$

and similarly for $y_{0}$. Thus, (4.2.18)-(4.2.20) and (1.23) show that each term in (4.2.16) and (4.2.17) is well defined. Inserting (4.2.17) into (4.2.16) results in a cancellation of the last two terms of (4.2.16), hence

$$
\begin{aligned}
\frac{d}{d \tau}\left(e^{A^{*}(\tau-t)} P(\tau) e^{A(\tau-t)} x, y\right)_{Y} & =-\left(R e^{A(\tau-t)} x, R e^{A(\tau-t)} y\right)_{Z} \\
+ & \left(B^{*} P(\tau) e^{A(\tau-t)} x, B^{*} P(\tau) e^{A(\tau-t)} y\right)_{U}, x, y \in Y_{\delta}^{-}
\end{aligned}
$$


Integrating (4.2.21) in $\tau$ over $[t, T]$ and using $P(T)=0$ from the D.R.E. (4.2.10) results in (4.2.15), as desired.

\section{Application: Kirchoff EQUATiOn With ONE BOUNDARY CONTROL. REGULARITY THEORY}

All dynamical abstract hypotheses $($ H.1 $)=(1.6)$ through $($ H.7 $)=(1.21)$ of Section 1 have already been shown to hold true in the following two cases:

(i) Second-order hyperbolic equations with Dirichlet boundary control, defined on a smooth, bounded domain $\Omega \subset \mathbf{R}^{n}$, see [L-T.3]; here one may take

$$
\begin{gathered}
U_{\theta} \equiv H^{\theta}(\Gamma) ; Y_{\theta} \equiv H_{0}^{\theta}(\Omega) \times H^{\theta-1}(\Omega), 0 \leq \theta \leq \frac{1}{2}+\delta, \theta \neq \frac{1}{2} \\
U_{0}=U=L_{2}(\Gamma) ; Y_{0}=Y=L_{2}(\Omega) \times H^{-1}(\Omega) \\
U_{\delta}^{-}=U_{\frac{1}{2}-\delta}=H^{\frac{1}{2}-\delta}(\Gamma) ; \quad U_{\delta}^{+}=U_{\frac{1}{2}+\delta}=H^{\frac{1}{2}+\delta}(\Gamma) \\
Y_{\delta}^{-}=Y_{\frac{1}{2}-\delta}=H^{\frac{1}{2}-\delta}(\Omega) \times H^{-\frac{1}{2}-\delta}(\Omega) \\
Y_{\delta}^{+}=Y_{\frac{1}{2}+\delta}=H_{0}^{\frac{1}{2}+\delta}(\Omega) \times H^{-\frac{1}{2}+\delta}(\Omega), \text { etc. }
\end{gathered}
$$

(ii) non-symmetric, non-dissipative, first-order hyperbolic systems with boundary control, see [C-L.1]; here one make take similarly defined explicit Sobolev spaces for $U_{\theta}, Y_{\theta}$, etc.

In this section we consider an optimal quadratic cost problem over a finite horizon for a Kirchoff equation, subject only to one control acting in the "moment" boundary condition. [The physical bending moment in the 2dimensional Kirchoff plate model is actually a modification of the boundary condition (5.1.1d) below.] The Kirchoff equation is hyperbolic with finite speed of propagation, and displays a behavior similar to that of the wave equation. In the case of the Kirchoff mixed problem, we shall show likewise that all abstract system's assumptions $($ H.1 $)=(1.6)$ through $($ H.7 $)=(1.21)$ of Section 1 are automatically satisfied in a natural mathematical setting. Many such settings can be chosen, and we shall select a particular interesting one where, as in the case of second-order hyperbolic equations of [L-T.5], the observation $R^{*} R$ jumps across a boundary condition, see (5.2.3)-(5.2.5) below. Accordingly, Theorems 2.1, 2.2, and 2.3 of Section 2 are then applicable to the present class, for any observation operator $R$ with "minimal" smoothing as in $($ H.8) $=(1.23)$. In a companion paper [T.1], we show that the Euler-Bernoulli equation [Eqn. (5.1.1a) below with $\rho=0$ ], which is not hyperbolic, also satisfies assumptions (H.1) through (H.7) in explicitly identified Sobolev spaces (different from the Kirchoff equation case). 


\subsection{Problem formulation}

The dynamics. Let $\Omega$ be an open bounded domain in $R^{n}$ with sufficiently smooth boundary $\Gamma$, say, of class $C^{2}$. The Kirchoff equation is given by

$$
\begin{cases}w_{t t}-\rho \Delta w_{t t}+\Delta^{2} w=0 & \text { in }(0, T] \times \Omega \equiv Q \\ w(0, \cdot)=w_{0}, w_{t}(0, \cdot)=w_{1} & \text { in } \Omega \\ \left.w\right|_{\Sigma}=0 & \text { in }(0, T] \times \Gamma \equiv \Sigma \\ \left.\Delta w\right|_{\Sigma}=u & \text { in } \Sigma,\end{cases}
$$

where $\rho>0$ is a constant (proportional to the square of the thickness in the 2-dimensional plate model), and where $u \in L_{2}\left(0, T ; L_{2}(\Gamma)\right) \equiv L_{2}(\Sigma)$ is the control function acting in the "moment" B.C. (5.1.1d).

The optimal control problem on $[s, T]$. Consistently with the (optimal) regularity theory for problem (5.1.1) presented in Theorem 5.3.2 below, the cost functional which we seek to minimize over all $u \in L_{2}\left(s, T ; L_{2}(\Gamma)\right)=$ $L_{2}\left(\Sigma_{s T}\right)$ is taken to be

$$
J(u, w)=\int_{0}^{T}\left\{\left\|R\left[\begin{array}{c}
w(t) \\
w_{t}(t)
\end{array}\right]\right\|_{H^{2}(\Omega) \times H^{1}(\Omega)}^{2}+\|u(t)\|_{L_{2}(\Gamma)}^{2}\right\} d t,
$$

with initial data $\left\{w_{0}, w_{1}\right\} \in\left[H^{2}(\Omega) \cap H_{0}^{1}(\Omega)\right] \times H_{0}^{1}(\Omega)$, where the observation operator $R \in \mathcal{L}\left(\left[H^{2}(\Omega) \cap H_{0}^{1}(\Omega)\right] \times H_{0}^{1}(\Omega)\right)$ will be further specified below in $(5.2 .3)$.

\subsection{MAIN RESULTS}

As a specialization to problem (5.1.1), (5.1.2) of the abstract theory presented in Theorems 2.1, 2.2, and 2.3 of Section 2, in the present section we establish the following results.

Theorem 5.2.1. (a) With the observation operator $R$ in (5.1.2) only assumed to satisfy

$$
R \in \mathcal{L}\left(\left[H^{2}(\Omega) \cap H_{0}^{1}(\Omega)\right] \times H_{0}^{1}(\Omega)\right)
$$

the preliminary theory of [L-T.3] applies to the optimal control problem (5.1.1), (5.1.2), with

$$
y(t)=\left[\begin{array}{c}
w(t) \\
w_{t}(t)
\end{array}\right] ; \quad U=L_{2}(\Gamma) ; Y \equiv\left[H^{2}(\Omega) \cap H_{0}^{1}(\Omega)\right] \times H_{0}^{1}(\Omega),
$$

and yields a unique optimal pair $\left\{u^{0}\left(\cdot, s ; y_{0}\right), y^{0}\left(\cdot, s ; y_{0}\right)\right\}, y_{0}=\left[w_{0}, w_{1}\right] \in$ $Y$, satisfying, in particular, the pointwise feedback synthesis, and the optimal cost relation of Remark 2.1, as well as the other properties listed in (2.1a), (2.2a).

(b) Assume, in addition to (5.2.1), that $R$ satisfies the smoothing assumption

$$
R^{*} R: \text { continuous } Y_{\delta}^{-} \rightarrow Y_{\delta}^{+}
$$


$R^{*}$ adjoint of $R$ in $Y$, where

$$
\begin{aligned}
Y_{\delta}^{-} & \equiv\left\{h \in H^{\frac{5}{2}-\delta}(\Omega):\left.h\right|_{\Gamma}=0\right\} \times\left\{h \in H^{\frac{3}{2}-\delta}(\Omega):\left.h\right|_{\Gamma}=0\right\} \\
& =\left[H^{\frac{5}{2}-\delta}(\Omega) \cap H_{0}^{1}(\Omega)\right] \times H^{\frac{3}{2}-\delta}(\Omega) \cap H_{0}^{1}(\Omega) \\
Y_{\delta}^{+} & \equiv\left\{h \in H^{\frac{5}{2}+\delta}(\Omega):\left.h\right|_{\Gamma}=\left.\Delta h\right|_{\Gamma}=0\right\} \times\left[H^{\frac{3}{2}+\delta}(\Omega) \cap H_{0}^{1}(\Omega)\right]
\end{aligned}
$$

(see Section 5.6), and where $\delta>0$ is an arbitrarily small constant, which is kept fixed throughout. [An additional characterization of $Y_{\delta}^{-}$and $Y_{\delta}^{+}$will be given below in $(5.6 .4),(5.6 .5)$ ]. Thus, the above choice centered at $\theta=\frac{5}{2}$ yields that assumption (5.2.3) jumps from $Y_{\delta}^{-}$to $Y_{\delta}^{+}$across the new B.C. $\left.\Delta h\right|_{\Gamma}=0$. (Refer to the paragraph before Section 5.1.) Then Theorems 2.1, 2.2 , and 2.3 of Section 2 hold true, with $Y_{\delta}^{-}$and $Y_{\delta}^{+}$given by (5.2.4) and (5.2.5), and with

$$
U_{\delta}^{-}=H^{\frac{1}{2}-\delta}(\Gamma) \quad U_{\delta}^{+}=H^{\frac{1}{2}+\delta}(\Gamma)
$$

(see Section 5.6). In particular, explicitly:

$\left(b_{1}\right)$ (regularity of the optimal pair) the optimal pair satisfies, for

$$
y_{0}=\left[w_{0}, w_{1}\right] \in\left[H^{\frac{5}{2}-\delta}(\Omega) \cap H_{0}^{1}(\Omega)\right] \times\left[H^{\frac{3}{2}-\delta}(\Omega) \cap H_{0}^{1}(\Omega)\right]
$$

the following regularity properties:

(i)

$$
\begin{aligned}
& {\left[\begin{array}{c}
w^{0}\left(\cdot, s ; y_{0}\right) \\
w_{t}^{0}\left(\cdot, s ; y_{0}\right)
\end{array}\right]=y^{0}\left(\cdot, s ; y_{0}\right)} \\
& \in C\left([s, T] ;\left[H^{\frac{5}{2}-\delta}(\Omega) \cap H_{0}^{1}(\Omega)\right] \times\left[H^{\frac{3}{2}-\delta}(\Omega) \cap H_{0}^{1}(\Omega)\right]\right) \\
& \cap \quad\left[H^{\frac{1}{2}-\delta}\left(s, T ;\left[H^{2}(\Omega) \cap H_{0}^{1}(\Omega)\right] \times H_{0}^{1}(\Omega)\right)\right.
\end{aligned}
$$

(ii)

$$
\begin{aligned}
u^{0}\left(\cdot, s ; y_{0}\right) & \in L_{2}\left(s, T ; H^{\frac{1}{2}+\delta}(\Gamma)\right) \cap H^{\frac{1}{2}+\delta}\left(s, T ; L_{2}(\Gamma)\right) \\
& \equiv H^{\frac{1}{2}+\delta, \frac{1}{2}+\delta}\left(\Sigma_{s T}\right)
\end{aligned}
$$

$\Sigma_{s T}=[s, T] \times \Gamma$, a fortiori,

$$
u^{0}\left(\cdot, s ; y_{0}\right) \in C\left([s, T] ; H^{\delta}(\Gamma)\right) .
$$

All the above results hold true uniformly in $s$, i.e., with norms which may be made independent of $s$. 
$\left(\mathrm{b}_{2}\right)$ (gain operator) The gain operator $B^{*} P(t), P(t)$ defined by $(2.3 \mathrm{a})$, satisfies the following regularity property (see $(2.4)$ ),

$$
\begin{aligned}
& B^{*} P(t) x= B^{*}\left[\begin{array}{ll}
P_{11}(t) & P_{12}(t) \\
P_{21}(t) & P_{22}(t)
\end{array}\right]\left[\begin{array}{l}
x_{1} \\
x_{2}
\end{array}\right] \\
&=-\frac{\partial}{\partial \nu}\left[P_{21}(t) x_{1}+P_{22}(t) x_{2}\right] \\
&: \quad \text { continuous }\left[H^{\frac{5}{2}-\delta}(\Omega) \cap H_{0}^{1}(\Omega)\right] \\
& \times\left[H^{\frac{3}{2}-\delta}(\Omega) \cap H_{0}^{1}(\Omega)\right] \rightarrow C\left([0, T] ; L_{2}(\Gamma)\right),
\end{aligned}
$$

where the adjoint $B^{*}$ is computed with respect to the space $Y$, topologized, however, as $Y \equiv \mathcal{D}\left(\mathcal{A}^{\frac{1}{2}}\right) \times \mathcal{D}\left(\mathcal{A}_{\rho}^{\frac{1}{4}}\right)$, see below in (5.4.22).

$\left(b_{3}\right)$ (Differential Riccati Equation) With

$$
A=\left[\begin{array}{cc}
0 & I \\
\mathbf{A} & 0
\end{array}\right], \quad \mathbf{A}=\left(I+\rho \mathcal{A}^{\frac{1}{2}}\right)^{-1} \mathcal{A}
$$

as in (5.4.3) below, we have that $P(t)$ satisfies the following D.R.E.

$$
\left\{\begin{array}{c}
\frac{d}{d t}(P(t) x, y)_{Y}=-(R x, R y)_{Y}-(P(t) x, A y)_{Y}-(P(t) A x, y)_{Y} \\
+\left(\frac{\partial}{\partial \nu}\left[P_{21}(t) x_{1}+P_{22}(t) x_{2}\right], \frac{\partial}{\partial \nu}\left[P_{21}(t) y_{1}+P_{22}(t) y_{2}\right]\right)_{L_{2}(\Gamma)} \\
P(T)=0, \forall x, y \in\left[H^{\frac{5}{2}-\delta}(\Omega) \cap H_{0}^{1}(\Omega)\right] \times\left[H^{\frac{3}{2}-\delta}(\Omega) \cap H_{0}^{1}(\Omega)\right] .
\end{array}\right.
$$

Furthermore, $P(t)$ satisfies the corresponding Integral Riccati Equation, as in $(2.7)$, for all such $x, y$.

\subsection{Regularity THEORY FOR PROBLEM (5.1.1) WITH $u \in L_{2}(\Sigma)$}

The following well-posedness Theorem 5.3.2 provides the critical regularity result, which justifies as natural the selection of the cost functional $J$ in (5.1.2), and which will permit us to verify assumption $(\mathrm{H} .1)=(1.6)$ below. To this end, as we shall see, it is expedient to associate with problem (5.1.1) the following boundary-homogeneous version (which would be the corresponding adjoint problem (5.5.7) below, if the initial conditions were given at $t=T$, an inessential modification since the problem is time-reversible),

$$
\begin{cases}\phi_{t t}-\rho \Delta \phi_{t t}+\Delta^{2} \phi=f & \text { in } Q \\ \phi(0, \cdot)=\phi_{0} ; \phi_{t}(0, \cdot)=\phi_{1} & \text { in } \Omega \\ \left.\phi\right|_{\Sigma} \equiv 0 ;\left.\Delta \phi\right|_{\Sigma} \equiv 0 & \text { in } \Sigma\end{cases}
$$

The following two key results are taken from [L-T.5]. 
Theorem 5.3.1. [L-T.5] With reference to (5.3.1), assume

$$
\begin{gathered}
\left\{\phi_{0}, \phi_{1}\right\} \in V \times\left[H^{2}(\Omega) \cap H_{0}^{1}(\Omega)\right] ; \quad f \in L_{1}\left(0, T ; L_{2}(\Omega)\right) ; \\
V=\left\{h \in H^{3}(\Omega):\left.h\right|_{\Gamma}=\left.\Delta h\right|_{\Gamma}=0\right\} .
\end{gathered}
$$

Then, the unique solution of problem (5.3.1) satisfies, continuously,

$$
\begin{gathered}
\left\{\phi, \phi_{t}\right\} \in C\left([0, T] ; V \times\left[H^{2}(\Omega) \times H_{0}^{1}(\Omega)\right]\right) ; \\
\phi_{t t} \in L_{1}\left(0, T ; H_{0}^{1}(\Omega)\right) ; \text { or } \phi_{t t} \in C\left([0, T] ; H_{0}^{1}(\Omega)\right) \text { if } f \equiv 0,
\end{gathered}
$$

and

$$
\frac{\partial(\Delta \phi)}{\partial \nu} \in L_{2}\left(0, T ; L_{2}(\Gamma)\right) \equiv L_{2}(\Sigma)
$$

Proof. The proof of ((5.3.5) of) Theorem 5.3.1 is by P.D.E.'s energy methods and will be indicated in Section 5.10 below.

Remark 5.3.1. The trace regularity (5.3.5) is, of course, the key result of Theorem 5.3.1. It does not follow from the optimal interior regularity (5.3.4): indeed, (5.3.5) is " $\frac{1}{2}$ higher" in Sobolev space regularity on $\Omega$ over a formal application of trace theory to $\phi$ in (5.3.4).

Theorem 5.3.2. [L-T.5] With reference to the non-homogeneous problem (5.1.1), assume

$$
\left\{w_{0}, w_{1}\right\} \in\left[H^{2}(\Omega) \cap H_{0}^{1}(\Omega)\right] \times H_{0}^{1}(\Omega) ; u \in L_{2}(\Sigma) .
$$

Then, the unique solution of (5.1.1) satisfies

$$
\begin{aligned}
\left.\left\{w, w_{t}\right\} \in C\left([0, T] ;\left[H^{2}(\Omega) \cap H_{0}^{1}(\Omega)\right]\right) \times H_{0}^{1}(\Omega)\right), & \\
& w_{t t} \in C\left([0, T] ; L_{2}(\Omega)\right)
\end{aligned}
$$

continuously; i.e., more precisely,

$$
\begin{aligned}
&\left\|\left\{w, w_{t}, w_{t t}\right\}\right\|_{\left.C\left([0, T] ;\left[H^{2}(\Omega) \cap H_{0}^{1}(\Omega)\right]\right) \times H_{0}^{1}(\Omega) \times L_{2}(\Omega)\right)}^{2} \\
& \leq C T\left\{\|u\|_{L_{2}(\Sigma)}^{2}+\left\|\left\{w_{0}, w_{1}\right\}\right\|_{\left[H^{2}(\Omega) \cap H_{0}^{1}(\Omega)\right] \times H_{0}^{1}(\Omega)}^{2}\right\}
\end{aligned}
$$

Proof. The proof of Theorem 5.3.2 is a consequence by transposition of Theorem 5.3.1 and will be given in Section 5.11 below.

\subsection{Abstract Setting For Problem (5.1.1)}

We follow [L-T.5], [L-T.6]. To put problems (5.1.1), (5.1.2) into the abstract model (1.1), (1.3), we introduce the following operators and spaces:

(i)

$$
\begin{aligned}
\mathcal{A} h & =\Delta^{2} h ; \mathcal{A}: \mathcal{D}(\mathcal{A}) \rightarrow L_{2}(\Omega) ; \\
\mathcal{D}(\mathcal{A}) & =\left\{h \in H^{4}(\Omega):\left.h\right|_{\Gamma}=\left.\Delta h\right|_{\Gamma}=0\right\} ;
\end{aligned}
$$




$$
\begin{aligned}
& \mathcal{A}^{\frac{1}{2}} h=-\Delta h ; \mathcal{D}\left(\mathcal{A}^{\frac{1}{2}}\right)=H^{2}(\Omega) \cap H_{0}^{1}(\Omega) ; \\
& \mathbf{A}=\left(I+\rho \mathcal{A}^{\frac{1}{2}}\right)^{-1} \mathcal{A} ; \mathcal{D}(\mathbf{A})=\mathcal{D}\left(\mathcal{A}^{\frac{1}{2}}\right) .
\end{aligned}
$$

The operator $\mathcal{A}$ in (5.4.1) is positive self-adjoint on $L_{2}(\Omega)$. Furthermore, as usual, we shall freely extend $\mathcal{A}$ originally defined in (5.4.1) [while maintaining the same symbol, with no fear of confusion] as $\mathcal{A}: L_{2}(\Omega) \rightarrow\left[\mathcal{D}\left(\mathcal{A}^{*}\right)\right]^{\prime}=$ $[\mathcal{D}(\mathcal{A})]^{\prime}$, duality with respect to the pivot space $L_{2}(\Omega)$. The following space identifications are known (with equivalent norms) [Gr.1], [B-D-D-M.1]

$$
\begin{aligned}
& \mathcal{D}\left(\mathcal{A}^{\theta}\right)=\left\{h \in H^{4 \theta}(\Omega):\left.h\right|_{\Gamma}=0\right\}, \quad \frac{1}{8}<\theta<\frac{5}{8} \\
& \mathcal{D}\left(\mathcal{A}^{\theta}\right)=\left\{h \in H^{4 \theta}(\Omega):\left.h\right|_{\Gamma}=\left.\Delta h\right|_{\Gamma}=0\right\}, \quad \frac{5}{8}<\theta \leq 1 .
\end{aligned}
$$

The following specializations thereof will be needed below:

$$
\begin{gathered}
\theta=\frac{1}{4}: \mathcal{D}\left(\mathcal{A}^{\frac{1}{4}}\right)=H_{0}^{1}(\Omega), \text { and for } g \in H_{0}^{1}(\Omega) \\
\|g\|_{\mathcal{D}\left(\mathcal{A}^{\frac{1}{4}}\right)}=\left\|\mathcal{A}^{\frac{1}{4}} g\right\|_{L_{2}(\Omega)}=\left\{\int_{\Omega}|\nabla g|^{2} d \Omega\right\}^{\frac{1}{2}} \\
\text { equivalent to the }\|g\|_{H_{0}^{1}(\Omega)^{-}}
\end{gathered}
$$

in turn equivalent to

$$
\begin{aligned}
\left\{\int_{\Omega}\left[g^{2}+\rho|\nabla g|^{2} d \Omega\right\}^{\frac{1}{2}}\right. & =\left\{\|g\|_{L_{2}(\Omega)}^{2}+\rho\left\|\mathcal{A}^{\frac{1}{4}} g\right\|_{L_{2}(\Omega)}^{2}\right\}^{\frac{1}{2}} \\
& \equiv\|g\|_{\mathcal{D}\left(\mathcal{A}_{\rho}^{\frac{1}{4}}\right)}=\|g\|_{H_{0, \rho}^{1}(\Omega)}
\end{aligned}
$$

the latter norm being denoted by $\mathcal{D}\left(\mathcal{A}_{\rho}^{\frac{1}{4}}\right)$-norm or $H_{0, \rho}^{1}(\Omega)$-norm;

$$
\theta=\frac{1}{2}: \mathcal{D}\left(\mathcal{A}^{\frac{1}{2}}\right)=\left\{h \in H^{2}(\Omega):\left.h\right|_{\Gamma}=0\right\}=H^{2}(\Omega) \cap H_{0}^{1}(\Omega),
$$

and for $g \in \mathcal{D}\left(\mathcal{A}^{\frac{1}{2}}\right)$ :

$$
\|g\|_{\mathcal{D}\left(\mathcal{A}^{\frac{1}{2}}\right)}=\left\|\mathcal{A}^{\frac{1}{2}} g\right\|_{L_{2}(\Omega)}=\left\{\int_{\Omega}(\Delta g)^{2} d \Omega\right\}^{\frac{1}{2}}
$$

equivalent to

$$
\left\{\left\|\mathcal{A}^{\frac{1}{4}} g\right\|_{L_{2}(\Omega)}^{2}+\rho\left\|\mathcal{A}^{\frac{1}{2}} g\right\|_{L_{2}(\Omega)}^{2}\right\}^{\frac{1}{2}}=\|g\|_{\mathcal{D}\left(\mathcal{A}_{\rho}^{\frac{1}{2}}\right)},
$$

the latter norm being denoted as $\mathcal{D}\left(\mathcal{A}_{\rho}^{\frac{1}{2}}\right)$-norm;

$$
\theta=\frac{3}{4}, \mathcal{D}\left(\mathcal{A}^{\frac{3}{4}}\right)=\left\{h \in H^{3}(\Omega):\left.h\right|_{\Gamma}=\left.\Delta h\right|_{\Gamma}=0\right\}
$$


and for $h \in \mathcal{D}\left(\mathcal{A}^{\frac{3}{4}}\right)$ :

$$
\|g\|_{\mathcal{D}\left(\mathcal{A}^{\frac{3}{4}}\right)}=\left\|\mathcal{A}^{\frac{3}{4}} g\right\|_{L_{2}(\Omega)}=\left\|\mathcal{A}^{\frac{1}{4}} \Delta g\right\|_{L_{2}(\Omega)}=\left\{\int_{\Omega}|\nabla(\Delta g)|^{2} d \Omega\right\}^{\frac{1}{2}},
$$

by (5.4.2) and (5.4.6b).

Problem (5.3.1) can then be rewritten abstractly as

$$
\begin{gathered}
\left(I+\rho \mathcal{A}^{\frac{1}{2}}\right) \phi_{t t}+\mathcal{A} \phi=f, \text { or } \phi_{t t}=-\mathbf{A} \phi+\left(I+\rho \mathcal{A}^{\frac{1}{2}}\right)^{-1} f, \\
\phi(0)=\phi_{0}, \phi_{t}(0)=\phi_{1},
\end{gathered}
$$

recalling (5.4.1)-(5.4.3). The operator in (5.4.3), rewritten by using twice $R\left(\lambda, \mathcal{A}^{\frac{1}{2}}\right) \mathcal{A}^{\frac{1}{2}}=-I+\lambda R\left(\lambda, \mathcal{A}^{\frac{1}{2}}\right)$ as

$$
\begin{aligned}
-\mathbf{A} & =-\left(I+\rho \mathcal{A}^{\frac{1}{2}}\right)^{-1} \mathcal{A}^{\frac{1}{2}} \mathcal{A}^{\frac{1}{2}}=-\left[\frac{1}{\rho} I-\frac{1}{\rho}\left(I+\rho \mathcal{A}^{\frac{1}{2}}\right)^{-1}\right] \mathcal{A}^{\frac{1}{2}} \\
& =-\frac{\mathcal{A}^{\frac{1}{2}}}{\rho}+\frac{1}{\rho^{2}} I-\frac{1}{\rho^{2}}\left(I+\rho \mathcal{A}^{\frac{1}{2}}\right)^{-1}
\end{aligned}
$$

$$
\mathbf{A}: \mathcal{D}(\mathbf{A})=\mathcal{D}\left(\mathcal{A}^{\frac{1}{2}}\right) \rightarrow L_{2}(\Omega),
$$

being a bounded perturbation of the negative self-adjoint operator $\mathcal{A}^{\frac{1}{2}}$, is the generator of a s.c. cosine operator $\mathbf{C}(t)$, with corresponding "sine" operator $\mathbf{S}(t)=\int_{0}^{t} \mathbf{C}(\tau) d \tau$, where the maps

$$
t \rightarrow \mathcal{A}^{\frac{1}{4}} \mathbf{S}(t), \mathbf{C}(t) \text {, are strongly continuous on } L_{2}(\Omega) \text {. }
$$

Accordingly, the unique solution of problem (5.4.9), or (5.3.1), is given explicitly by:

$$
\begin{gathered}
\phi(t)=\mathbf{C}(t) \phi_{0}+\mathbf{S}(t) \phi_{1}+\int_{0}^{t} \mathbf{S}(t-\tau)\left(I+\rho \mathcal{A}^{\frac{1}{2}}\right)^{-1} f(\tau) d \tau, \\
\phi_{t}(t)=-\mathbf{A} \mathbf{S}(t) \phi_{0}+\mathbf{C}(t) \phi_{1}+\int_{0}^{t} \mathbf{C}(t-\tau)\left(I+\rho \mathcal{A}^{\frac{1}{2}}\right)^{-1} f(\tau) d \tau,
\end{gathered}
$$

in appropriate function spaces, depending on $\left\{\phi_{0}, \phi_{1}, f\right\}$.

Moreover, returning to (5.4.3),

A is a positive self-adjoint operator on the space $\mathcal{D}\left(\mathcal{A}_{\rho}^{\frac{1}{4}}\right)$ defined by $(5.4 .6 \mathrm{c})$, with respect to the corresponding inner product.

$$
(x, y)_{\mathcal{D}\left(\mathcal{A}_{\rho}^{\frac{1}{4}}\right)}=\left(\left(I+\rho \mathcal{A}^{\frac{1}{2}}\right) x, y\right)_{L_{2}(\Omega)}, \quad x, y \in H_{0}^{1}(\Omega) .
$$

(ii) We introduce the Green map $G_{2}$ by

$$
y=G_{2} v \Longleftrightarrow\left\{\Delta^{2} y=0 \text { in } \Omega ;\left.y\right|_{\Gamma}=0 ;\left.\Delta y\right|_{\Gamma}=v\right\},
$$

and by elliptic theory [L-M.1, vol. I, p. 188]

$$
G_{2}: H^{s}(\Gamma) \rightarrow H^{s+\frac{5}{2}}(\Omega), \quad s \in \mathbf{R} .
$$


We have already shown in [L-T.4]-[L-T.6], that

$$
G_{2}=-\mathcal{A}^{-\frac{1}{2}} D \text { where } y=D v \Longleftrightarrow\left\{\Delta y=0 \text { in } \Omega ;\left.y\right|_{\Gamma}=v\right\},
$$

$D$ being the Dirichlet map satisfying the regularity,

$$
\begin{gathered}
D: \text { continuous } H^{s}(\Gamma) \rightarrow H^{s+\frac{1}{2}}(\Omega) ; \text { in particular, } \\
D: \text { continuous } L_{2}(\Gamma) \rightarrow H^{\frac{1}{2}}(\Omega) \subset H^{\frac{1}{2}-2 \epsilon}(\Omega) \\
=\mathcal{D}\left(\mathcal{A}^{\frac{1}{8}-\frac{\epsilon}{2}}\right), \epsilon>0 ; \\
\mathcal{A}^{\frac{1}{8}-\frac{\epsilon}{2}} D \in \mathcal{L}\left(L_{2}(\Gamma) ; L_{2}(\Omega)\right) ; D^{*} \mathcal{A}^{\frac{1}{8}-\frac{\epsilon}{2}} \in \mathcal{L}\left(L_{2}(\Omega) ; L_{2}(\Gamma)\right),
\end{gathered}
$$

with the property,

$$
G_{2}^{*} \mathcal{A} h=-D^{*} \mathcal{A}^{\frac{1}{2}} h=-\frac{\partial}{\partial \nu} h, \quad h \in \mathcal{D}(\mathcal{A}) .
$$

(iii) By (5.4.15), (5.4.1), problem (5.1.1) can be written abstractly, first as

$$
\begin{array}{r}
\left(I+\rho \mathcal{A}^{\frac{1}{2}}\right) w_{t t}+\mathcal{A}\left(w-G_{2} u\right)=0 \text { on } L_{2}(\Omega), \\
\text { or } w_{t t}=-\mathbf{A} w+\mathbf{A} G_{2} u \text { on }[\mathcal{D}(\mathcal{A})]^{\prime},
\end{array}
$$

recalling (5.4.3), next as in (1.1); i.e., as

$$
\begin{gathered}
y_{t}=A y+B u \text { on }\left[\mathcal{D}\left(A^{*}\right)\right]^{\prime}, \quad y(0)=y_{0} \in Y ; \\
Y \equiv \mathcal{D}\left(\mathcal{A}^{\frac{1}{2}}\right) \times \mathcal{D}\left(\mathcal{A}_{\rho}^{\frac{1}{4}}\right)=\left[H^{2}(\Omega) \cap H_{0}^{1}(\Omega)\right] \times H_{0}^{1}(\Omega) ; \\
U=L_{2}(\Gamma)(\text { equivalent norms); } \\
y=\left[\begin{array}{c}
w \\
w_{t}
\end{array}\right] ; \quad A=\left[\begin{array}{cc}
0 & I \\
-\mathbf{A} & 0
\end{array}\right], \mathcal{D}(A)=\mathcal{D}\left(\mathcal{A}^{\frac{3}{4}}\right) \times \mathcal{D}\left(\mathcal{A}^{\frac{1}{2}}\right) \rightarrow Y ; \\
\left.B u=\left[\begin{array}{c}
0 \\
\mathbf{A} G_{2} u
\end{array}\right]: \text { continuous } U \rightarrow\left[\mathcal{D}\left(A^{*}\right)\right]^{\prime} \text { (duality w.r.t. } Y\right),
\end{gathered}
$$

equivalently,

$$
A^{-1} B \text { : continuous } U \rightarrow Y,
$$

since, in fact, for $u \in L_{2}(\Gamma)$, recalling (5.4.23) and (5.4.17),

$$
\begin{gathered}
A^{-1} B u=\left[\begin{array}{cc}
0 & -\mathbf{A}^{-1} \\
I & 0
\end{array}\right]\left[\begin{array}{c}
0 \\
\mathbf{A} G_{2} u
\end{array}\right]=\left[\begin{array}{c}
-G_{2} u \\
0
\end{array}\right]=\left[\begin{array}{c}
\mathcal{A}^{-\frac{1}{2}} D u \\
0
\end{array}\right] \in Y \\
\mathcal{A}^{-\frac{1}{2}} D u \in \mathcal{D}\left(\mathcal{A}^{\frac{5}{8}-\frac{\epsilon}{2}}\right) .
\end{gathered}
$$

It is property (5.4.14) for $\mathbf{A}$ that makes the choice of $\mathcal{D}\left(\mathcal{A}_{\rho}^{\frac{1}{4}}\right)$ as the second component space of $Y$ particularly convenient. In fact, with such a choice, we have that $A$ in (5.4.23) is skew-adjoint on $Y=\mathcal{D}\left(\mathcal{A}^{\frac{1}{2}}\right) \times \mathcal{D}\left(\mathcal{A}_{\rho}^{\frac{1}{4}}\right)$, i.e., $A^{*}=-A$, and so it generates a s.c. unitary group $e^{A t}$ on $Y$. 
Starting from (5.4.24) we compute $B^{*}$ with respect to $Y$ topologized as $\mathcal{D}\left(\mathcal{A}^{\frac{1}{2}}\right) \times \mathcal{D}\left(\mathcal{A}_{\rho}^{\frac{1}{4}}\right)$, to obtain for $v=\left[v_{1}, v_{2}\right]$, recalling (5.4.14):

$$
\begin{aligned}
(B u, v)_{Y} & =\left(\mathbf{A} G_{2} u, v_{2}\right)_{\mathcal{D}\left(\mathcal{A}_{\rho}^{\frac{1}{4}}\right)}=\left(\mathcal{A} G_{2} u, v_{2}\right)_{L_{2}(\Omega)} \\
& =\left(u, G_{2}^{*} \mathcal{A} v_{2}\right)_{L_{2}(\Gamma)}
\end{aligned}
$$

i.e., by virtue of (5.4.19):

$$
B^{*}\left[\begin{array}{l}
v_{1} \\
v_{2}
\end{array}\right]=G_{2}^{*} \mathcal{A} v_{2}=-\frac{\partial v_{2}}{\partial \nu}: \mathcal{D}\left(A^{*}\right)=\mathcal{D}(A) \rightarrow U .
$$

Eqn. (5.4.22), the solution of problem (5.1.1), or (5.4.21), with initial condition $y(s)=y_{0}=\left[w_{0}, w_{1}\right]$ is written abstractly as

$$
\begin{gathered}
{\left[\begin{array}{c}
w\left(t, s ; y_{0}\right) \\
w_{t}\left(t, s ; y_{0}\right.
\end{array}\right]=e^{A(t-s)}\left[\begin{array}{c}
w_{0} \\
w_{1}
\end{array}\right]+\left(L_{s} u\right)(t) ;} \\
\left(L_{s} u\right)(t)=\int_{s}^{t} e^{A(t-\tau)} B u(\tau) d \tau=\left[\begin{array}{c}
\left.\mathbf{A} \int_{s}^{t} \mathbf{S}(t-\tau) G_{2} u(\tau) d \tau\right] \\
\mathbf{A} \int_{s}^{t} \mathbf{C}(t-\tau) G_{2} u(\tau) d \tau
\end{array}\right] \\
=\left[\begin{array}{c}
-\left(I+\rho \mathcal{A}^{\frac{1}{2}}\right)^{-1} \mathcal{A}^{\frac{1}{2}} \int_{s}^{t} \mathbf{S}(t-\tau) D u(\tau) d \tau \\
\left.-\left(I+\rho \mathcal{A}^{\frac{1}{2}}\right)^{-1} \mathcal{A}^{\frac{1}{2}} \mathbf{A} \int_{s}^{t} \mathbf{C}(t-\tau) D u(\tau) d \tau\right]
\end{array}\right.
\end{gathered}
$$

after recalling, in the last step, (5.4.3) and (5.4.17), where $A$ generates a s.c. group $e^{A t}$ on $Y, t \in R$, which is given by

$$
e^{A t}=\left[\begin{array}{cc}
\mathbf{C}(t) & \mathbf{S}(t) \\
-\mathbf{A S}(t) & \mathbf{C}(t)
\end{array}\right]
$$

In (5.4.31), $\mathbf{C}(t)$ is even and $\mathbf{S}(t)$ is odd. By (5.4.28) and (5.4.31), since $A$ is skew-adjoint on $Y, A^{*}=-A$, and so $e^{A^{*} t}=e^{-A t}$, we compute with $x=\left[x_{1}, x_{2}\right] \in Y=\mathcal{D}\left(\mathcal{A}^{\frac{1}{2}}\right) \times \mathcal{D}\left(\mathcal{A}_{\rho}^{\frac{1}{4}}\right):$

$$
\begin{aligned}
B^{*} e^{A^{*} t} x & =G_{2}^{*} \mathcal{A}\left[\mathbf{A} \mathbf{S}(t) x_{1}+\mathbf{C}(t) x_{2}\right] \\
& =G_{2}^{*} \mathcal{A} \mathcal{A}^{\frac{1}{2}}\left[\mathbf{C}(t) \mathcal{A}^{-\frac{1}{2}} x_{2}+\mathbf{S}(t)\left(I+\rho \mathcal{A}^{\frac{1}{2}}\right)^{-1} \mathcal{A}^{\frac{1}{2}} x_{1}\right]
\end{aligned}
$$

$($ by $(5.4 .28))=G_{2}^{*} \mathcal{A} \mathcal{A}^{\frac{1}{2}}\left[\mathbf{C}(t) \phi_{0}+\mathbf{S}(t) \phi_{1}\right]=-\frac{\partial}{\partial \nu} \Delta \phi\left(t ; \phi_{0}, \phi_{1}\right)$, recalling (5.4.28), (5.4.2), and (5.4.12), where $\phi\left(t ; \phi_{0}, \phi_{1}\right)$ solves problem (5.3.1) with $f \equiv 0$ and

$$
\begin{aligned}
& \phi_{0}=\mathcal{A}^{-\frac{1}{2}} x_{2} \in \mathcal{D}\left(\mathcal{A}^{\frac{3}{4}}\right), \text { for } x_{2} \in \mathcal{D}\left(\mathcal{A}^{\frac{1}{4}}\right)=\mathcal{D}\left(\mathcal{A}_{\rho}^{\frac{1}{4}}\right) \\
& \phi_{1}=\left(I+\rho \mathcal{A}^{\frac{1}{2}}\right)^{-1} \mathcal{A}^{\frac{1}{2}} x_{1} \in \mathcal{D}\left(\mathcal{A}^{\frac{1}{2}}\right) \text { for } x_{1} \in \mathcal{D}\left(\mathcal{A}^{\frac{1}{2}}\right) .
\end{aligned}
$$


From (5.4.34)-(5.4.36), we see that $($ H.1) $=(1.6)$ holds true

$$
\int_{0}^{T}\left\|B^{*} e^{A^{*} t} x\right\|_{U}^{2} d t \leq C_{T}\|x\|_{Y}^{2}, \quad x \in Y,
$$

if and only if problem (5.3.1) with $f \equiv 0$ satisfies

$$
\int_{0}^{T} \int_{\Gamma}\left(\frac{\partial \Delta \phi}{\partial \nu}\right)^{2} d \Sigma \leq C_{T}\left\|\left\{\phi_{0}, \phi_{1}\right\}\right\|_{\mathcal{D}\left(\mathcal{A}^{\frac{3}{4}}\right) \times \mathcal{D}\left(\mathcal{A}^{\frac{1}{2}}\right)}^{2},
$$

which is precisely the trace regularity result, guaranteed by Theorem 5.3.1, Eqn. (5.3.5). Then, according to duality [L-T.2]-[L-T.6], estimate (5.5.1), i.e., (5.5.2), is, in turn, equivalent to the following property that

$$
\begin{gathered}
\left(L_{s} u\right)(t)=\int_{s}^{t} e^{A(t-\tau)} B u(\tau) d \tau=\left[\begin{array}{c}
w(t ; 0,0) \\
w_{t}(t ; 0,0)
\end{array}\right] \\
\quad: \text { continuous } L_{2}\left(s, T ; L_{2}(\Gamma)\right) \\
\rightarrow C\left([s, T] ; \mathcal{D}\left(\mathcal{A}^{\frac{1}{2}}\right) \times \mathcal{D}\left(\mathcal{A}^{\frac{1}{4}}\right) \equiv\left[H^{2}(\Omega) \cap H_{0}^{1}(\Omega)\right] \times H_{0}^{1}(\Omega)\right)
\end{gathered}
$$

uniformly in $s$, where $w_{0}=w_{1}=0$ in problem (5.1.1). This is precisely conclusion (5.3.7) of Theorem 5.3.2, the additional statement of uniformity in $s$ being an immediate consequence of formula (5.5.3a) for $L_{s}$ (via a change of variable). Moreover, recalling (5.4.33), we have, by duality on (5.5.3) with $v=\left[v_{1}, v_{2}\right]$ :

$$
\begin{aligned}
&\left(L_{s}^{*} v\right)(t)= B^{*} \int_{t}^{T} e^{A^{*}(\tau-t)} v(\tau) d \tau, \quad s \leq \tau \leq T \\
&(\operatorname{by}(5.4 .33))= G_{2}^{*} \mathcal{A}^{\frac{1}{2}}\left\{\int_{t}^{T} \mathbf{S}(\tau-t)\left(I+\rho \mathcal{A}^{\frac{1}{2}}\right)^{-1} \mathcal{A}^{\frac{1}{2}} v_{1}(\tau) d \tau\right. \\
&\left.+\int_{t}^{T} \mathbf{C}(\tau-t) \mathcal{A}^{-\frac{1}{2}} v_{2}(\tau) d \tau\right\} \\
&: \text { continuous } L_{1}\left(s, T ; \mathcal{D}\left(\mathcal{A}^{\frac{1}{2}}\right) \times \mathcal{D}\left(\mathcal{A}^{\frac{1}{4}}\right)\right) \\
& \rightarrow L_{2}\left(s, T ; L_{2}(\Gamma)\right)
\end{aligned}
$$

uniformly in $s$. Now, let $\psi(t ; h)$ be the solution of the (adjoint) problem

$$
\begin{cases}\psi_{t t}-\rho \Delta \psi_{t t}+\Delta^{2} \psi=f & \text { in } Q ; \\ \psi(T, \cdot)=0 ; \psi_{t}(T ; \cdot)=0 & \text { in } \Omega ; \\ \left.\left.\psi\right|_{\Sigma} \equiv \Delta \psi\right|_{\Sigma} \equiv 0 & \text { in } \Sigma,\end{cases}
$$

rewritten abstractly via (5.4.3) as

$$
\psi_{t t}=-\mathbf{A} \psi+h ; \quad h=\left(I+\rho \mathcal{A}^{\frac{1}{2}}\right)^{-1} f ; \quad \psi(T)=\psi_{t}(T)=0,
$$


hence given explicitly by

$$
\psi(t ; h)=\int_{T}^{t} \mathbf{S}(t-\tau) h(\tau) d \tau ; \psi_{t}(t ; h)=\int_{T}^{t} \mathbf{C}(t-\tau) h(\tau) d \tau .
$$

Then, recalling (5.4.19) and (5.4.2), we see via (5.5.9) that the two terms in (5.5.5) can be rewritten, with

$$
v=\left[v_{1}, v_{2}\right] \in L_{1}\left(0, T ; \mathcal{D}\left(\mathcal{A}^{\frac{1}{2}}\right) \times \mathcal{D}\left(\mathcal{A}^{\frac{1}{4}}\right)\right),
$$

as

$$
\begin{aligned}
\left(L_{s}^{*} v\right)(t)= & \frac{\partial \Delta}{\partial \nu}\left\{\int_{T}^{t} \mathbf{S}(t-\tau) h_{1}(\tau) d \tau-\int_{T}^{t} \mathbf{C}(t-\tau) h_{2}(\tau) d \tau\right\} \\
= & \frac{\partial \Delta \psi\left(t ; h_{1}\right)}{\partial \nu}-\frac{\partial \Delta \psi_{t}\left(t ; h_{2}\right)}{\partial \nu} \\
& \quad \text { continuous } v=\left[v_{1}, v_{2}\right] \in L_{1}\left(0, T ; \mathcal{D}\left(\mathcal{A}^{\frac{1}{2}}\right) \times \mathcal{D}\left(\mathcal{A}^{\frac{1}{4}}\right)\right) \\
& \rightarrow L_{2}\left(0, T ; L_{2}(\Gamma)\right) \\
h_{1} & =\left(I+\rho \mathcal{A}^{\frac{1}{2}}\right)^{-1} \mathcal{A}^{\frac{1}{2}} v_{1} \in L_{1}\left(0, T ; \mathcal{D}\left(\mathcal{A}^{\frac{1}{2}}\right)\right) \\
h_{2} & =\mathcal{A}^{-\frac{1}{2}} v_{2} \in L_{1}\left(0, T ; \mathcal{D}\left(\mathcal{A}^{\frac{3}{4}}\right)\right)
\end{aligned}
$$

Notice that regularity (5.5.11) of the normal trace $\frac{\partial \Delta}{\partial \nu} \psi\left(t ; h_{1}\right)$, for $\psi\left(t ; h_{1}\right)$ solution of (5.5.8) due to $h=h_{1}$ given by (5.5.12), is precisely conclusion (5.3.5) of Theorem 5.3.1 for the time-reversed problem $\psi$ in (5.3.1) with initial data at $t=0$, rather than $t=T$ as for $\psi$, an inessential modification. The proof of Theorem 5.3.1 in [L-T.5] is by energy (P.D.E.'s)-methods. Instead, regularity (5.5.9) for the normal trace $\frac{\partial \Delta}{\partial \nu} \psi_{t}\left(t ; h_{2}\right)$ for the time derivative $\psi_{t}$ of the solution $\psi\left(t ; h_{2}\right)$ of problem (5.5.8) due to $h=h_{2}$ given by (5.5.13) is obtained by duality via operator methods as in (5.5.6)-(5.5.11) [while it appears that purely P.D.E. methods will require a time regularity assumption of the right-hand side, non-homogeneous term]. Thus, assumption (H.1) = (1.6) is verified.

\subsection{Selection of spaces $U_{\theta}$ ANd $Y_{\theta}$ IN (1.10)}

We select the spaces in (1.10) to be the following Sobolev spaces

$$
\begin{gathered}
U_{\theta}=H^{\theta}(\Gamma) ; U_{0}=U=L_{2}(\Gamma), \quad 0 \leq \theta \leq \frac{1}{2}+\delta, \theta \neq \frac{1}{2} ; \\
Y_{\theta}=\mathcal{D}\left(\mathcal{A}^{\frac{1}{2}+\frac{\theta}{4}}\right) \times \mathcal{D}\left(\mathcal{A}^{\frac{1}{4}+\frac{\theta}{4}}\right) \equiv \mathcal{D}\left(A^{\theta}\right) \\
Y_{0}=Y=\mathcal{D}\left(\mathcal{A}^{\frac{1}{2}}\right) \times \mathcal{D}\left(\mathcal{A}^{\frac{1}{4}}\right)
\end{gathered}
$$


where $A$ is as in (5.4.23), in particular the critical spaces for $\theta=\frac{1}{2} \pm \delta$ :

$$
\begin{aligned}
& U_{\delta}^{-}=U_{\frac{1}{2}-\delta}=H^{\frac{1}{2}-\delta}(\Gamma) ; \quad U_{\delta}^{+}=U_{\frac{1}{2}+\delta}=H^{\frac{1}{2}+\delta}(\Gamma) \\
& Y_{\delta}^{-}=Y_{\frac{1}{2}-\delta}=\mathcal{D}\left(A^{\frac{1}{2}-\delta}\right)=\mathcal{D}\left(A^{\frac{5}{8}-\frac{\delta}{4}}\right) \times \mathcal{D}\left(A^{\frac{3}{8}-\frac{\delta}{4}}\right) \\
& =\left[H^{\frac{5}{2}-\delta}(\Omega) \cap H_{0}^{1}(\Omega)\right] \times\left[H^{\frac{3}{2}-\delta}(\Omega) \cap H_{0}^{1}(\Omega)\right] \\
& Y_{\delta}^{+}=Y_{\frac{1}{2}+\delta}=\mathcal{D}\left(A^{\frac{1}{2}+\delta}\right)=\mathcal{D}\left(A^{\frac{5}{8}+\frac{\delta}{4}}\right) \times \mathcal{D}\left(A^{\frac{3}{8}+\frac{\delta}{4}}\right) \\
& =\left\{h \in H^{\frac{5}{2}+\delta}(\Omega):\left.h\right|_{\Gamma}=\left.\Delta h\right|_{\Gamma}=0\right\} \times\left[H^{\frac{3}{2}+\delta}(\Omega) \cap H_{0}^{1}(\Omega)\right],
\end{aligned}
$$

recalling (5.4.4) and, respectively, (5.4.5), with equivalent norms. The spaces $\left[Y_{\delta}^{-}\right]^{\prime}$ and $\left[Y_{\delta}^{+}\right]^{\prime}$, duality with respect to $Y=\mathcal{D}\left(\mathcal{A}^{\frac{1}{2}}\right) \times \mathcal{D}\left(\mathcal{A}^{\frac{1}{4}}\right)$, are given by

$$
\begin{aligned}
{\left[Y_{\delta}^{-}\right]^{\prime} } & =\mathcal{D}\left(\mathcal{A}^{\frac{3}{8}+\frac{\delta}{4}}\right) \times \mathcal{D}\left(\mathcal{A}^{\frac{1}{8}+\frac{\delta}{4}}\right) \\
& =\left[H^{\frac{3}{2}+\delta}(\Omega) \cap H_{0}^{1}(\Omega)\right] \times H_{0}^{\frac{1}{2}+\delta}(\Omega) \\
{\left[Y_{\delta}^{+}\right]^{\prime} } & =\mathcal{D}\left(\mathcal{A}^{\frac{3}{8}-\frac{\delta}{4}}\right) \times \mathcal{D}\left(\mathcal{A}^{\frac{1}{8}-\frac{\delta}{4}}\right) \\
& =\left[H^{\frac{3}{2}-\delta}(\Omega) \cap H_{0}^{1}(\Omega)\right] \times H^{\frac{1}{2}-\delta}(\Omega) .
\end{aligned}
$$

Thus, by (5.6.4) and (5.6.7) we verify the interpolation property

$$
\left[Y_{\delta}^{-},\left[Y_{\delta}^{+}\right]^{\prime}\right]_{\theta=\frac{1}{2}-\delta}=Y=\mathcal{D}\left(\mathcal{A}^{\frac{1}{2}}\right) \times \mathcal{D}\left(\mathcal{A}^{\frac{1}{4}}\right)
$$

as required in $(1.10)$, since $\left(\frac{5}{8}-\frac{\delta}{4}\right)(1-\theta)+\left(\frac{3}{8}-\frac{\delta}{4}\right) \theta=\frac{1}{2}$ for the first component; and $\left(\frac{3}{8}-\frac{\delta}{4}\right)(1-\theta)+\left(\frac{1}{8}-\frac{\delta}{4}\right) \theta=\frac{1}{4}$ for $\theta=\frac{1}{2}-\delta$, for the second component space. Moreover, the injections $U_{\theta_{1}} \hookrightarrow U_{\theta_{2}}, Y_{\theta_{1}} \hookrightarrow Y_{\theta_{2}}$ are compact, $0 \leq \theta_{2}<\theta_{1} \leq \frac{1}{2}+\delta$, as required in (1.10), since $\Omega$ is a bounded domain. Thus, the spaces in (1.11), (1.12) are in the present case as follows for $0 \leq \theta \leq \frac{1}{2}+\delta, \theta \neq \frac{1}{2}$ :

$$
\begin{aligned}
& \mathcal{U}^{\theta}[s, T]= H^{\theta, \theta}\left(\Sigma_{s T}\right) \\
&= L_{2}\left(s, T ; H^{\theta}(\Gamma)\right) \cap H^{\theta}\left(s, T ; L_{2}(\Gamma)\right) \\
& \mathcal{Y}^{\theta}[s, T]= L_{2}\left(s, T ; \mathcal{D}\left(\mathcal{A}^{\frac{1}{2}+\frac{\theta}{4}}\right) \times \mathcal{D}\left(\mathcal{A}^{\frac{1}{4}+\frac{\theta}{4}}\right)\right) \\
& \cap H^{\theta}\left(s, T ; \mathcal{D}\left(\mathcal{A}^{\frac{1}{2}}\right) \times \mathcal{D}\left(\mathcal{A}^{\frac{1}{4}}\right)\right) \\
&= L_{2}\left(s, T ; \mathcal{D}\left(A^{\theta}\right)\right) \cap H^{\theta}(s, T ; Y) \\
& \mathcal{D}\left(A^{\theta}\right)=\mathcal{D}\left(\mathcal{A}^{\frac{1}{2}+\frac{\theta}{4}}\right) \times \mathcal{D}\left(\mathcal{A}^{\frac{1}{4}+\frac{\theta}{4}}\right) .
\end{aligned}
$$




\subsection{Verification of ASSUMption $(\mathrm{H} .2)=(1.14)$}

The following regularity result is critical in verifying assumption (H.2) = (1.14). It is the main new P.D.E. result of this paper.

Theorem 5.7.1. With reference to the non-homogeneous problem (5.1.1), assume

$$
\left\{\begin{array}{c}
w_{0} \in H^{3}(\Omega) \cap H_{0}^{1}(\Omega), w_{1} \in H^{2}(\Omega) \cap H_{0}^{1}(\Omega) ; \\
\text { with the compatibility relations } \\
\left.w_{0}\right|_{\Gamma}=0 \text { and }\left.\Delta w_{0}\right|_{\Gamma}=u(0) ; \\
u \in C\left([0, T] ; H^{\frac{1}{2}}(\Gamma)\right) \cap H^{1}\left(0, T ; L_{2}(\Gamma)\right)
\end{array}\right.
$$

$\left[(5.7 .2)\right.$ is a fortiori guaranteed, if $u \in H^{1,1}(\Sigma)$, by [L-M.1, I, Thm. 3.1, p. $19]$.

Then, the unique solution to problem (5.1.1) satisfies

$$
\begin{aligned}
& \left\{w, w_{t}, w_{t t}\right\} \\
& \quad \in C\left([0, T] ;\left[H^{3}(\Omega) \cap H_{0}^{1}(\Omega)\right] \times\left[H^{2}(\Omega) \cap H_{0}^{1}(\Omega)\right] \times H_{0}^{1}(\Omega)\right),
\end{aligned}
$$

continuously.

Proof. The proof of Theorem 5.7.1 will be given in Section 5.12 below.

Corollary 5.7.2. With reference to the non-homogeneous problem (5.1.1), assume $w_{0}=w_{1}=0$, and for $0 \leq \theta<\frac{1}{2}$ :

$$
u \in H^{\theta, \theta}(\Sigma)=\mathcal{U}^{\theta}[0, T]=L_{2}\left(0, T ; H^{\theta}(\Gamma)\right) \cap H^{\theta}\left(0, T ; L_{2}(\Gamma)\right) .
$$

Then, the unique solution to problem (5.1.1) satisfies

$$
\begin{aligned}
& \left\{\begin{array}{l}
w(\cdot ; 0,0) \in C\left([0, T] ; \mathcal{D}\left(\mathcal{A}^{\frac{1}{2}+\frac{\theta}{4}}\right)=H^{2+\theta}(\Omega) \cap H_{0}^{1}(\Omega)\right) ; \\
w_{t}(\cdot ; 0,0) \in C\left([0, T] ; \mathcal{D}\left(\mathcal{A}^{\frac{1}{4}+\frac{\theta}{4}}\right)=H^{1+\theta}(\Omega) \cap H_{0}^{1}(\Omega)\right) ; \\
w_{t t}(\cdot ; 0,0) \in C\left([0, T] ; \mathcal{D}\left(\mathcal{A}^{\frac{\theta}{4}}\right)=H^{\theta}(\Omega)\right)
\end{array}\right. \\
& \left\{\begin{array}{l}
D_{t}^{r} w(\cdot ; 0,0) \in L_{2}\left(0, T ; \mathcal{D}\left(\mathcal{A}^{\frac{1}{2}+\frac{\theta}{4}-\frac{r}{4}}\right)\right), 0 \leq r \leq 1 ; \\
D_{t}^{\theta} w(\cdot ; 0,0) \in L_{2}\left(0, T ; \mathcal{D}\left(\mathcal{A}^{\frac{1}{2}}\right)\right) ;
\end{array}\right. \\
& \left\{\begin{array}{l}
D_{t}^{r} w_{t}(\cdot ; 0,0) \in L_{2}\left(0, T ; \mathcal{D}\left(\mathcal{A}^{\frac{1}{4}+\frac{\theta}{4}-\frac{r}{4}}\right)\right), 0 \leq r \leq 1 ; \\
D_{t}^{\theta} w_{t}(\cdot ; 0,0) \in L_{2}\left(0, T ; \mathcal{D}\left(\mathcal{A}^{\frac{1}{4}}\right)\right)
\end{array}\right.
\end{aligned}
$$


A fortiori,

$$
\begin{aligned}
L u \in \mathcal{Y}^{\theta}[0, T]= & L_{2}\left(0, T ; \mathcal{D}\left(\mathcal{A}^{\frac{1}{2}+\frac{\theta}{4}}\right) \times \mathcal{D}\left(\mathcal{A}^{\frac{1}{4}+\frac{\theta}{2}}\right)=\mathcal{D}\left(A^{\theta}\right)\right) \\
& \cap H^{\theta}\left(0, T ; \mathcal{D}\left(\mathcal{A}^{\frac{1}{2}}\right) \times \mathcal{D}\left(\mathcal{A}^{\frac{1}{4}}\right)=Y\right) .
\end{aligned}
$$

Proof of Corollary 5.7.2. For $\theta<\frac{1}{2}$, the compatibility relations in (5.7.1), which now read $u(0)=\left.\Delta w_{0}\right|_{\Gamma}=0 ;\left.w_{0}\right|_{\Gamma}=0$, do not interfere, and we then interpolate between $(5.3 .6),(5.3 .7)$, or $(5.5 .3 \mathrm{~b})$, for $\theta=0$ and (5.7.2), (5.7.3) for $\theta=1$, thereby obtaining (5.7.5)-(5.7.7), as desired.

Next, application of the intermediate derivative theorem [L-M.1, p. 15] to (5.7.5) and (5.7.6), as well as to (5.7.6) and (5.7.7), yields, respectively, (5.7.8) and (5.7.10), which then specialize to (5.7.9), and respectively, (5.7.11) for $r=\theta$. Thus, (5.7.12) is a consequence of (5.7.5), (5.7.6), and (5.7.9), (5.7.11).

Corollary 5.7.2 plainly verifies assumption $($ H.2) $=(1.1 .4)$.

\subsection{VERIFICATION OF ASSUMPTION $($ H.3) $=(1.15)$}

Verification of assumption (H.3) $=(1.15)$ is based upon the following regularity result.

Theorem 5.8.1. (i) With reference to the operator $L^{*}$ defined by (5.5.4), we have for $0 \leq r \leq 1$,

$$
\begin{gathered}
\left(L_{s}^{*} v\right)(t)=\quad B^{*} \int_{t}^{T} e^{A^{*}(\tau-t)} v(\tau) d \tau, \quad s \leq t \leq T \\
: \text { continuous } L_{2}\left(s, T ; \mathcal{D}\left(\mathcal{A}^{\frac{1}{2}+\frac{r}{4}}\right) \times \mathcal{D}\left(\mathcal{A}^{\frac{1}{4}+\frac{r}{4}}\right) \equiv \mathcal{D}\left(A^{r}\right)\right) \\
\rightarrow H^{r, r}\left(\Sigma_{s T}\right)=\mathcal{U}^{r}[s, T]
\end{gathered}
$$

uniformly in $s$.

Proof. The proof of Theorem 5.8.1 will be given in Section 5.13 below.

Restricting (5.8.1) to $0 \leq \theta=r \leq \frac{1}{2}+\delta, \theta \neq \frac{1}{2}$, we obtain verification of assumption $(\mathrm{H} .3)=(1.15)$.

\subsection{Verification of assumptions $($ H.4 $)=(1.18)$ \\ THROUGH $($ H.7) $=(1.21)$}

Verification of assumption (H.4) $=$ (1.18). For $x=\left[x_{1}, x_{2}\right] \in Y_{\delta}^{+}=$ $\mathcal{D}\left(A^{\frac{1}{2}+\delta}\right)=\mathcal{D}\left(\mathcal{A}^{\frac{5}{8}+\frac{\delta}{4}}\right) \times \mathcal{D}\left(\mathcal{A}^{\frac{3}{8}+\frac{\delta}{4}}\right)$ by $(5.6 .5)$, we compute starting from 
(5.4.33), and recalling $G_{2}^{*} \mathcal{A}=-D^{*} \mathcal{A}^{-\frac{1}{2}}$ from (5.4.17):

$$
\begin{gathered}
B^{*} e^{A^{*} t} x=G_{2}^{*} \mathcal{A}\left[\mathbf{C}(t) x_{2}+\mathbf{S}(t)\left(I+\rho \mathcal{A}^{\frac{1}{2}}\right)^{-1} \mathcal{A} x_{1}\right] \\
(\text { by }(5.4 .17))=-D^{*} \mathcal{A}^{\frac{1}{2}}\left[\mathbf{C}(t) x_{2}+\mathbf{S}(t)\left(I+\rho \mathcal{A}^{\frac{1}{2}}\right)^{-1} \mathcal{A} x_{1}\right] \\
=-D^{*} \mathcal{A}^{\frac{1}{8}-\frac{\delta}{4}}\left[\mathbf{C}(t) \mathcal{A}^{\frac{3}{8}+\frac{\delta}{4}} x_{2}+\mathcal{A}^{\frac{1}{4}} \mathbf{S}(t)\left(I+\rho \mathcal{A}^{\frac{1}{2}}\right)^{-1} \mathcal{A}^{\frac{9}{8}+\frac{\delta}{4}} x_{1}\right] \\
\in C\left([0, T] ; L_{2}(\Gamma)\right),
\end{gathered}
$$

where the desired regularity in (5.9.4) follows since, recalling (5.4.18c) and (5.4.11),

$$
\begin{array}{r}
D^{*} \mathcal{A}^{\frac{1}{8}-\frac{\delta}{4}} \in \mathcal{L}\left(L_{2}(\Omega) ; L_{2}(\Gamma)\right) ; \quad t \rightarrow \mathcal{A}^{\frac{1}{4}} \mathbf{S}(t), \\
\mathbf{C}(t) \text { strongly continuous on } L_{2}(\Omega),
\end{array}
$$

as well as, via the assumptions on $\left[x_{1}, x_{2}\right]$.

$$
\mathcal{A}^{\frac{3}{8}+\frac{\delta}{4}} x_{2} \in L_{2}(\Omega) ;\left(I+\rho \mathcal{A}^{\frac{1}{2}}\right)^{-1} \mathcal{A}^{\frac{9}{8}+\frac{\delta}{4}} x_{1} \in L_{2}(\Omega) .
$$

Thus, (5.9.4) verifies assumption $($ H.4) $=(1.18)$.

Verification of assumption (H.5) $=$ (1.19). With

$$
Y_{\delta}^{-}=\mathcal{D}\left(\mathcal{A}^{\frac{5}{8}-\frac{\delta}{4}}\right) \times \mathcal{D}\left(\mathcal{A}^{\frac{3}{8}-\frac{\delta}{4}}\right)
$$

by (5.6.4), $\mathbf{C}(t)$ and $\mathbf{S}(t)$ are likewise s.c. cosine/sine operators on any space $\mathcal{D}\left(\mathcal{A}^{\theta}\right)$, hence $e^{A t}$ in (5.4.31) is a s.c. group on $Y_{\delta}^{-}$as well. The space $\mathcal{D}(A)$ in (5.4.23) is clearly dense in $Y_{\delta}^{-}$.

Verification of assumption (H.6) $=\mathbf{( 1 . 2 0 )}$. For $x=\left[x_{1}, x_{2}\right] \in Y_{\delta}^{-}=$ $\mathcal{D}\left(\mathcal{A}^{\frac{5}{8}-\frac{\delta}{4}}\right) \times \mathcal{D}\left(\mathcal{A}^{\frac{3}{8}-\frac{\delta}{4}}\right)=\mathcal{D}\left(A^{\frac{1}{2}-\delta}\right)$, we obtain via (5.4.23), (5.4.3),

$$
\begin{aligned}
A x & =\left[\begin{array}{ccc}
0 & I \\
-\left(I+\rho \mathcal{A}^{\frac{1}{2}}\right)^{-1} \mathcal{A} & 0
\end{array}\right]\left[\begin{array}{l}
x_{1} \\
x_{2}
\end{array}\right] \\
& =\left[\begin{array}{c}
x_{2} \\
-\left(I+\rho \mathcal{A}^{\frac{1}{2}}\right)^{-1} \mathcal{A} x_{1}
\end{array}\right] \in \mathcal{D}\left(\mathcal{A}^{\frac{3}{8}-\frac{\delta}{4}}\right) \times \mathcal{D}\left(\mathcal{A}^{\frac{1}{8}-\frac{\delta}{4}}\right) \equiv\left[Y_{\delta}^{+}\right]^{\prime},
\end{aligned}
$$

recalling in the last step (5.6.7) [and $A$ is, in fact, an isomorphism $Y_{\delta}^{-}$onto $\left.\left[Y_{\delta}^{+}\right]^{\prime}\right]$. Eqn. (5.9.7) verifies assumption $($ H.6) $=(1.20)$.

Remark 5.9.1. Returning to (5.4.26), we see via (5.6.4) that, in the present case,

$$
A^{-1} B \text { : continuous } U \rightarrow Y_{\delta}^{-},
$$

which is property (1.32). Thus, as remarked below (1.32), property (5.9.8), along with $($ H.5 $)=(1.19)$ and $($ H.6) $=(1.20)$ already verified, reprove $($ H.4 $)$ $=(1.18)$. 
Verification of assumption $($ H.7) $=(1.21)$. Let

$$
u \in U_{\delta}^{-}=H^{\frac{1}{2}-\delta}(\Gamma)
$$

so that

$$
D u \in H^{1-\delta}(\Omega),
$$

by (5.4.18a). Thus, recalling (5.4.3), (5.4.24), and (5.4.17),

$$
\begin{aligned}
B u & =\left[\begin{array}{c}
0 \\
\mathbf{A} G_{2} u
\end{array}\right]=\left[\begin{array}{c}
0 \\
-\left(I+\rho \mathcal{A}^{\frac{1}{2}}\right)^{-1} \mathcal{A}^{\frac{1}{2}} D u
\end{array}\right] \subset\left[Y_{\delta}^{+}\right]^{\prime} \\
& =\left[H^{\frac{3}{2}-\delta}(\Omega) \cap H_{0}^{1}(\Omega)\right] \times H^{\frac{1}{2}-\delta}(\Omega),
\end{aligned}
$$

using, in the last step, (5.6.7) for $\left[Y_{\delta}^{+}\right]^{\prime}$, and (5.9.9). Thus, (5.9.10) shows

$$
B: \text { continuous } U_{\delta}^{-} \rightarrow\left[Y_{\delta}^{+}\right]^{\prime},
$$

as desired, and assumption (H.7) $=(1.21)$ is verified.

\subsection{Proof of (5.3.5) OF THEOREM 5.3.1}

Key to this end is the following result. It is reported here because it will be critically invoked in Section 5.13.

Lemma 5.10.1. [L-T.3] Let $\phi$ be a solution of Eqn. (5.3.1a) (with no boundary conditions imposed) for smooth data, say

$$
\left\{\phi_{0}, \phi_{1}, f\right\} \in \mathcal{D}(\mathcal{A}) \times \mathcal{D}\left(\mathcal{A}^{\frac{3}{4}}\right) \times L_{1}\left(0, T ; \mathcal{D}\left(\mathcal{A}^{\frac{1}{4}}\right)\right) .
$$

Then, the following identity holds true:

$$
\begin{aligned}
\int_{\Sigma} \frac{\partial(\Delta \phi)}{\partial \nu} h \cdot \nabla(\Delta \phi) d \Sigma+\int_{\Sigma} \frac{\partial \phi_{t}}{\partial \nu} h \cdot \nabla \phi_{t} d \Sigma \\
+\frac{\rho}{2} \int_{\Sigma}\left(\Delta \phi_{t}\right)^{2} h \cdot \nu d \Sigma-\frac{1}{2} \int_{\Sigma}|\nabla(\Delta \phi)|^{2} h \cdot \nu d \Sigma-\frac{1}{2} \int_{\Sigma}\left|\Delta \phi_{t}\right|^{2} h \cdot \nu d \Sigma \\
\quad+\int_{\Sigma} \frac{\partial \phi_{t}}{\partial \nu} \phi_{t} \operatorname{div} h d \Sigma-\int_{\Sigma} \phi_{t} \Delta \phi_{t} h \cdot \nu d \Sigma \\
=\quad \int_{Q} H \nabla(\Delta \phi) \cdot \nabla(\Delta \phi) d Q+\int_{Q} H \nabla \phi_{t} \cdot \nabla \phi_{t} d Q \\
\quad+\frac{1}{2} \int_{Q}\left\{\left|\nabla \phi_{t}\right|^{2}+\rho\left(\Delta \phi_{t}\right)^{2}-|\nabla(\Delta \phi)|^{2}\right\} \operatorname{div} h d Q \\
\quad+\int_{Q} \phi_{t} \nabla(\operatorname{div} h) \cdot \nabla \phi_{t} d Q+\int_{Q} f h \cdot \nabla(\Delta \phi) d Q \\
\quad-\left[\left(\phi_{t}, h \cdot \nabla(\Delta \phi)\right)_{\Omega}+\rho\left(\Delta \phi_{t}, h \cdot \nabla(\Delta \phi)\right)_{\Omega}\right]_{0}^{T}
\end{aligned}
$$


where

$$
\begin{aligned}
& H(x)=\left[\begin{array}{c}
\frac{\partial h_{1}}{\partial x_{1}}, \cdots \frac{\partial h_{1}}{\partial x_{n}} \\
\cdots \\
\frac{\partial h_{n}}{\partial x_{1}}, \cdots \frac{\partial h_{n}}{\partial x_{n}}
\end{array}\right], \\
& \nu(x)=\text { outward unit normal vector at } x \in \Gamma,
\end{aligned}
$$

and $h(x)=\left[h_{1}(x), h_{2}(x), \ldots, h_{n}(x)\right] \in C^{2}(\bar{\Omega})$ is a given vector field.

Proof of Lemma 5.10.1. The key is to multiply Eqn. (5.3.1a) by $h$. $\nabla(\Delta \phi)$ and integrate by parts over $Q$, see [L-T.5].

\subsection{Proof of theorem 5.3.2}

We now provide the details, already contained in the preceding development, that the trace regularity for the homogeneous $\phi$-problem (5.3.1),

$$
\begin{aligned}
\left.\begin{array}{l}
\left\{\phi_{0}, \phi_{1}\right\} \in \mathcal{D}\left(\mathcal{A}^{\frac{3}{4}}\right) \times \mathcal{D}\left(\mathcal{A}^{\frac{1}{2}}\right) \\
f \equiv 0
\end{array}\right\} \Rightarrow \frac{\partial(\Delta \phi)}{\partial \nu} & \in L_{2}\left(0, T ; L_{2}(\Gamma)\right) \\
& \equiv L_{2}(\Sigma),
\end{aligned}
$$

established in Theorem 5.3.1, implies by transposition the interior regularity

$$
\begin{aligned}
& \left.\begin{array}{l}
u \in L_{2}\left(0, T ; L_{2}(\Gamma)\right) \equiv L_{2}(\Sigma) \\
w_{0}=w_{1}=0
\end{array}\right\} \Rightarrow \\
& {\left[\begin{array}{c}
w(t) \\
w_{t}(t)
\end{array}\right]=\left[\begin{array}{c}
\mathbf{A} \int_{0}^{t} \mathbf{S}(t-\tau) G_{2} u(\tau) d \tau \\
\mathbf{A} \int_{0}^{t} \mathbf{C}(t-\tau) G_{2} u(\tau) d \tau
\end{array}\right] \in C([0, T] ; Y) ;} \\
& Y=\mathcal{D}\left(\mathcal{A}^{\frac{1}{2}}\right) \times \mathcal{D}\left(\mathcal{A}_{\rho}^{\frac{1}{4}}\right)=\left[H^{2}(\Omega) \cap H_{0}^{1}(\Omega)\right] \times H_{0}^{1}(\Omega)
\end{aligned}
$$

(see (5.4.30) and (5.4.22)) for the non-homogeneous $w$-problem (5.1.1).

Operator-theoretic proof of Theorem 5.3.2. Step 1. We have already seen in Section 5.4, Eqns. (5.4.32)-(5.4.36), that

$$
\begin{gathered}
-\frac{\partial \Delta \phi}{\partial \nu}\left(t ; \phi_{0}, \phi_{1}\right)=G_{2}^{*} \mathbf{A}\left[\mathbf{S}(t) \mathcal{A} x_{1}+\mathbf{C}(t)\left(I+\rho \mathcal{A}^{\frac{1}{2}}\right) x_{2}\right] \\
=B^{*} e^{A^{*} t} x ; \\
\phi_{0}=\mathcal{A}^{-\frac{1}{2}} x_{2} ; \quad \phi_{1}=\left(I+\rho \mathcal{A}^{\frac{1}{2}}\right)^{-1} \mathcal{A}^{\frac{1}{2}} x_{1} .
\end{gathered}
$$

Thus, if we take

$$
\begin{array}{r}
x_{1} \in \mathcal{D}\left(\mathcal{A}^{\frac{1}{2}}\right), x_{2} \in \mathcal{D}\left(\mathcal{A}^{\frac{1}{4}}\right)=\mathcal{D}\left(\mathcal{A}_{\rho}^{\frac{1}{4}}\right), \text { hence } \\
\left\{\phi_{0}, \phi_{1}\right\} \in \mathcal{D}\left(\mathcal{A}^{\frac{3}{4}}\right) \times \mathcal{D}\left(\mathcal{A}^{\frac{1}{2}}\right),
\end{array}
$$


we then see, via (5.11.4), (5.11.5), that implication (5.11.1) of Theorem 5.3.1 applies and yields the following operator-theoretic restatement, already noted in (5.5.1).

Theorem 5.11.1. With reference to (5.11.4)-(5.11.6), (5.11.1), we have with $Y$ as in (5.11.3) and $\mathbf{A}$ as in (5.4.3):

$$
\begin{aligned}
& B^{*} e^{A^{*} t}: \text { continuous } Y \rightarrow L_{2}\left(0, T ; L_{2}(\Gamma)\right) \equiv L_{2}(\Sigma) \\
& G_{2}^{*} \mathbf{A} \mathcal{A}^{\frac{1}{2}} \mathbf{S}(t), G_{2}^{*} \mathcal{A} \mathbf{S}(t): \text { continuous } L_{2}(\Omega) \rightarrow L_{2}(\Sigma) ; \\
& G_{2}^{*} \mathbf{A} \mathcal{A}^{\frac{1}{4}} \mathbf{C}(t) ; G_{2}^{*} \mathcal{A}^{\frac{3}{4}} \mathbf{C}(t): \text { continuous } L_{2}(\Omega) \rightarrow L_{2}(\Sigma) .
\end{aligned}
$$

Step 2. The following result then stems from Theorem 5.11.1, by an application of [L-T.3] or [F-L-T.1, Appendix A]. With reference to the (operator) explicit formulas (5.4.29), (5.4.30) for the solution $\left\{w(t), w_{t}(t)\right\}$ of the $w$-problem (5.1.1) with initial conditions $w_{0}=w_{1}=0$ at $t=s=0$, we have

$$
\begin{aligned}
{\left[\begin{array}{c}
w(t) \\
w_{t}(t)
\end{array}\right]=} & (L u)(t)=\int_{0}^{t} e^{A(t-\tau)} B u(\tau) d \tau \\
= & {\left[\begin{array}{c}
\mathbf{A} \int_{0}^{t} \mathbf{S}(t-\tau) G_{2} u(\tau) d \tau \\
\mathbf{A} \int_{0}^{t} \mathbf{C}(t-\tau) G_{2} u(\tau) d \tau
\end{array}\right] } \\
& : \text { continuous } L_{2}(\Sigma) \rightarrow C([0, T] ; Y),
\end{aligned}
$$

$Y$ as in (5.11.3). This establishes (5.11.2) and, in turn, yields the key part of Theorem 5.3.2 due to $u$. Then, recalling (5.4.20), (5.4.3), (5.4.17), we obtain

$$
\begin{aligned}
w_{t t} & =-\mathbf{A} w+\mathbf{A} G_{2} u \\
& =\left(I+\rho \mathcal{A}^{\frac{1}{2}}\right)^{-1} \mathcal{A} w-\left(I+\rho \mathcal{A}^{\frac{1}{2}}\right)^{-1} \mathcal{A}^{\frac{1}{2}} D u \\
& \in L_{2}\left(0, T ; L_{2}(\Omega)\right),
\end{aligned}
$$

where the indicated regularity stems from the established regularity $w \in C\left([0, T] ; \mathcal{D}\left(\mathcal{A}^{\frac{1}{2}}\right)\right)$ of $w$ in (5.11.12), and by the regularity of $D$ in (5.4.18b). To get $w_{t t} \in L_{2}\left(0, T ; L_{2}(\Omega)\right)$, we could also differentiate $w_{t}$ in (5.11.1). Finally, we omit the details for the regularity due to the initial conditions $\left\{w_{0}, w_{1}\right\}$, using (5.4.11). Theorem 5.3.2 is proved.

A P.D.E. version of the duality or transposition argument may be given. 


\subsection{Proof of TheOREM 5.7.1}

With reference to the non-homogeneous $w$-problem (5.1.1), we assume

$$
\begin{array}{r}
\left\{w_{0}, w_{1}\right\} \in\left[H^{3}(\Omega) \cap H_{0}^{1}(\Omega)\right] \times\left[H^{2}(\Omega) \cap H_{0}^{1}(\Omega)\right] ; \\
\left.w_{0}\right|_{\Gamma}=0 ;\left.\quad \Delta w_{0}\right|_{\Gamma}=u(0) \in H^{\frac{1}{2}}(\Gamma) ; \\
u \in C\left([0, T] ; H^{\frac{1}{2}}(\Gamma)\right) \cap H^{1}\left(0, T ; L_{2}(\Gamma)\right),
\end{array}
$$

and we must show that

$$
\begin{aligned}
& \left\{w, w_{t}, w_{t t}\right\} \\
& \quad \in C\left([0, T] ;\left[H^{3}(\Omega) \cap H_{0}^{1}(\Omega)\right] \times\left[H^{2}(\Omega) \cap H_{0}^{1}(\Omega)\right] \times H_{0}^{1}(\Omega)\right) .
\end{aligned}
$$

Operator-theoretic proof. We return to the explicit solution formula (5.4.30), i.e.,

$$
w(t)=\mathbf{C}(t) w_{0}+\mathbf{S}(t) w_{1}+\mathbf{A} \int_{0}^{t} \mathbf{S}(t-\tau) G_{2} u(\tau) d \tau,
$$

and integrate by parts the integral term with $u \in H^{1}\left(0, T ; L_{2}(\Gamma)\right)$, thus obtaining

$$
w(t)=\mathbf{C}(t)\left[w_{0}-G_{2} u(0)\right]+\mathbf{S}(t) w_{1}+G_{2} u(t)-\int_{0}^{t} \mathbf{C}(t-\tau) G_{2} \dot{u}(\tau) d \tau .
$$

Here, by the first Compatibility Condition in (5.12.1), and, respectively, $\dot{u} \in L_{2}(\Sigma)$, we have

$$
w_{0}=G_{2} u(0) ; \quad \int_{0}^{t} \mathbf{C}(t-\tau) G_{2} \dot{u}(\tau) d \tau \in C\left([0, T] ; \mathcal{D}\left(\mathcal{A}^{\frac{3}{4}}\right)\right),
$$

recalling the definition of $G_{2}$ in (5.4.15) and, respectively, the regularity (5.11.2) (second component) $\left[\mathbf{A} z \in \mathcal{D}\left(\mathcal{A}^{\frac{1}{4}}\right) \Leftrightarrow z \in \mathcal{D}\left(\mathcal{A}^{\frac{3}{4}}\right)\right]$. Likewise, by (5.4.11),

$$
\mathbf{S}(t) w_{1} \in C\left([0, T] ; \mathcal{D}\left(\mathcal{A}^{\frac{3}{4}}\right)\right), \text { with } w_{1} \in \mathcal{D}\left(\mathcal{A}^{\frac{1}{2}}\right) .
$$

We now use that $u \in C\left([0, T] ; H^{\frac{1}{2}}(\Gamma)\right)$ as well, from (5.12.2), so that by elliptic theory [(5.4.15), (5.4.16) with $\left.s=\frac{1}{2}\right]$,

$$
G_{2} u(t) \in C\left([0, T] ; H^{3}(\Omega) \cap H_{0}^{1}(\Omega)\right),
$$

since $\left.G_{2} u(t)\right|_{\Gamma}=0$ by definition (5.4.15). Thus, (5.12.6), (5.12.7), and (5.12.8) used in (5.12.5), show (5.12.3) for $w$, via (5.4.8a). As to $w_{t}$, we differentiate (5.12.5), thus obtaining

$$
\begin{aligned}
w_{t}(t) & =\mathbf{C}(t) w_{1}+G_{2} \dot{u}(t)-G_{2} \dot{u}(t)+\mathbf{A} \int_{0}^{t} \mathbf{S}(t-\tau) G_{2} \dot{u}(\tau) d \tau \\
& \in C\left([0, T] ; \mathcal{D}\left(\mathcal{A}^{\frac{1}{2}}\right)\right) .
\end{aligned}
$$


Conclusion (5.12.3) for $w_{t}$ now follows from (5.12.9) [where a cancellation of $G_{2} \dot{u}(t)$ occurs] via (5.11.2) [first component] and (5.11.3). Similarly, one differentiates (5.12.9) in $t$ and obtains (5.12.3) for $w_{t t}$ via (5.11.2) [second component] and (5.11.3).

A P.D.E. proof may also be given, of course.

\subsection{Proof of theOREM 5.8.1}

\subsubsection{A PRELIMINARY TRACE RESUlt}

Theorem 5.13.1. (i) With reference to the operators in (5.11.8), (5.11.9), we have the following regularity properties for $0 \leq r \leq 1$ which generalize the case $r=0$ of Theorem 5.11.1:

$$
\begin{gathered}
G_{2}^{*} \mathbf{A} \mathcal{A}^{\frac{1}{2}} \mathbf{S}(t), \quad G_{2}^{*} \mathcal{A} \mathbf{S}(t): \text { continuous } \mathcal{D}\left(\mathcal{A}^{\frac{r}{4}}\right) \rightarrow H^{r, r}(\Sigma) \\
G_{2}^{*} \mathbf{A} \mathcal{A}^{\frac{1}{4}} \mathbf{C}(t), \quad G_{2}^{*} \mathcal{A}^{\frac{3}{4}} \mathbf{C}(t): \text { continuous } \mathcal{D}\left(\mathcal{A}^{\frac{r}{4}}\right) \rightarrow H^{r, r}(\Sigma) \\
B^{*} e^{A^{*} t}: \text { continuous } \mathcal{D}\left(A^{r}\right) \equiv \mathcal{D}\left(\mathcal{A}^{\frac{1}{2}+\frac{r}{4}}\right) \times \mathcal{D}\left(\mathcal{A}^{\frac{1}{4}+\frac{r}{4}}\right) \rightarrow H^{r, r}(\Sigma) \\
H^{r, r}(\Sigma) \equiv L_{2}\left(0, T ; H^{r}(\Gamma)\right) \cap H^{r}\left(0, T ; L_{2}(\Gamma)\right)
\end{gathered}
$$

(ii) Equivalently, in P.D.E.'s terms (see (5.3.5) for $r=0$ )

$$
\begin{array}{r}
\left\{\phi_{0}, \phi_{1}\right\} \rightarrow \frac{\partial \Delta \phi\left(t ; \phi_{0}, \phi_{1}\right)}{\partial \nu}: \text { continuous } \\
\mathcal{D}\left(A^{1+r}\right)=\mathcal{D}\left(\mathcal{A}^{\frac{3}{4}+\frac{r}{4}}\right) \times \mathcal{D}\left(\mathcal{A}^{\frac{1}{2}+\frac{r}{4}}\right) \rightarrow H^{r, r}(\Sigma),
\end{array}
$$

where $\phi\left(t ; \phi_{0}, \phi_{1}\right)$ is the solution of problem (5.3.1) with $f \equiv 0$, and where we further recall (5.11.4)-(5.11.6) to justify the stated equivalence between parts (i) and (ii).

Proof. Case $r=0$. The case $r=0$ is contained in Theorem 5.11.1 for (i) and in Theorem 5.3.1, Eqn. (5.3.5), for (ii). The stated equivalence uses (5.11.4)-(5.11.6). Thus, it is sufficient to prove the case $r=1$, and interpolate to establish Theorem 5.13.1.

Case $r=1$. We first show the time regularity

$$
G_{2}^{*} \mathbf{A} \mathcal{A}^{\frac{1}{2}} \mathbf{S}(t) x, G_{2}^{*} \mathbf{A} \mathcal{A}^{\frac{1}{4}} \mathbf{C}(t) x \in H^{1}\left(0, T ; L_{2}(\Gamma)\right), x \in \mathcal{D}\left(\mathcal{A}^{\frac{1}{4}}\right) .
$$

Indeed, with $x \in \mathcal{D}\left(\mathcal{A}^{\frac{1}{4}}\right)$, we compute, recalling from (5.4.9) and f.f. that $-\mathbf{A}$ is the infinitesimal generator of $\mathbf{C}(t)$

$$
\begin{aligned}
\frac{d}{d t} G_{2}^{*} \mathbf{A} \mathcal{A}^{\frac{1}{2}} \mathbf{S}(t) x & =G_{2}^{*} \mathbf{A} \mathcal{A}^{\frac{1}{4}} \mathbf{C}(t) \mathcal{A}^{\frac{1}{4}} x \in L_{2}(\Sigma) \\
\frac{d}{d t} G_{2}^{*} \mathbf{A} \mathcal{A}^{\frac{1}{4}} \mathbf{C}(t) x & =-G_{2}^{*} \mathbf{A}^{2} \mathbf{S}(t) \mathcal{A}^{\frac{1}{4}} x \in L_{2}(\Sigma),
\end{aligned}
$$

where the regularity in (5.13.8) is a direct application of (5.11.10) (case $r=0)$, while the regularity of (5.13.9) is equivalent to (5.11.9) (case $r=0)$ 
by the definition of $\mathbf{A}$ in (5.4.3). Thus, (5.13.7) is proved. To show the space regularity

$$
G_{2}^{*} \mathbf{A} \mathcal{A}^{\frac{1}{2}} \mathbf{S}(t) x, G_{2}^{*} \mathbf{A} \mathcal{A}^{\frac{1}{4}} \mathbf{C}(t) x \in L_{2}\left(0, T ; H^{1}(\Gamma)\right), x \in \mathcal{D}\left(\mathcal{A}^{\frac{1}{4}}\right),
$$

we shall equivalently show, by (5.11.4)-(5.11.6) that

$$
\begin{aligned}
\left\{\phi_{0}, \phi_{1}\right\} & \rightarrow \frac{\partial \Delta \phi\left(t ; \phi_{0}, \phi_{1}\right)}{\partial \nu}: \text { continuous } \mathcal{D}\left(A^{2}\right) \\
= & \mathcal{D}(\mathcal{A}) \times \mathcal{D}\left(\mathcal{A}^{\frac{3}{4}}\right) \rightarrow L_{2}\left(0, T ; H^{1}(\Gamma)\right)
\end{aligned}
$$

with $\phi\left(t ; \phi_{0}, \phi_{1}\right)$ solutions of problem (5.3.1) with $f \equiv 0$. To this end, we introduce

$$
\left\{\begin{array}{l}
\mathcal{B}=\sum_{i} b_{i}(x) \frac{\partial}{\partial x_{i}}=\text { first-order operator with } \\
\text { (time independent) coefficients } b_{i} \text { smooth in } \bar{\Omega} \text { and such that } \\
\mathcal{B} \text { is tangent to } \Gamma \text {, i.e., } \sum_{i} b_{i} \nu_{i}=0 \text { on } \Gamma .
\end{array}\right.
$$

Accordingly, we consider the problem

$$
\begin{cases}\phi_{t t}-\rho \Delta \phi_{t t}+\Delta^{2} \phi \equiv 0 & \text { in } Q \\ \phi(0, \cdot)=\phi_{0} ; \phi_{t}(0, \cdot)=\phi_{1} & \text { in } \Omega \quad \text { or } \phi_{t t}=-\mathbf{A} \phi \\ \left.\left.\phi\right|_{\Sigma} \equiv \Delta \phi\right|_{\Sigma} \equiv 0 & \text { in } \Sigma \\ \left\{\phi_{0}, \phi_{1}\right\} \in \mathcal{D}(\mathcal{A}) \times \mathcal{D}\left(\mathcal{A}^{\frac{3}{4}}\right) \subset H^{4}(\Omega) \times H^{3}(\Omega),\end{cases}
$$

whose solution is

$$
\begin{aligned}
\phi(t) & =\mathbf{C}(t) \phi_{0}+\mathbf{S}(t) \phi_{1} \in C([0, T] ; \mathcal{D}(\mathcal{A})) \\
\phi_{t}(t) & =-\mathbf{A} \mathbf{S}(t) \phi_{0}+\mathbf{C}(t) \phi_{1} \in C\left([0, T] ; \mathcal{D}\left(\mathcal{A}^{\frac{3}{4}}\right)\right) ; \\
\phi_{t t}(t) & =-\mathbf{A C}(t) \phi_{0}-\mathbf{A} \mathbf{S}(t) \phi_{1} \in C\left([0, T] ; \mathcal{D}\left(\mathcal{A}^{\frac{1}{2}}\right)\right) .
\end{aligned}
$$

We then introduce a new variable

$$
z=\mathcal{B} \phi,
$$

which, therefore, has a-priori regularity from (5.13.12) and (5.13.15)-(5.13.17), given by

$$
\left\{\begin{aligned}
z & \in C\left([0, T] ; H^{3}(\Omega) \cap H_{0}^{1}(\Omega)\right) ; \\
z_{t} & \in C\left([0, T] ; H^{2}(\Omega) \cap H_{0}^{1}(\Omega)\right) ; \\
z_{t t} & \in C\left([0, T] ; H_{0}^{1}(\Omega)\right) .
\end{aligned}\right.
$$

Then proving (5.13.11) is equivalent to showing that

$$
\left.\frac{\partial(\Delta \mathcal{B} \phi)}{\partial \nu}\right|_{\Gamma}=\left.\frac{\partial(\Delta z)}{\partial \nu}\right|_{\Gamma} \in L_{2}(\Sigma) .
$$


The variable $z$ satisfies the problem

$$
\begin{cases}z_{t t}-\rho \Delta z_{t t}+\Delta^{2} z=-\left[\mathcal{B}, \Delta^{2}\right] \phi+\rho[\mathcal{B}, \Delta] \phi_{t t} & \text { in } Q \\ \left.z\right|_{\Sigma} \equiv 0 & \text { in } \Sigma \\ \left.\Delta z\right|_{\Sigma}=-\left.[\mathcal{B}, \Delta] \phi\right|_{\Gamma} & \text { in } \Sigma\end{cases}
$$

as one readily sees by $(5.3 .18),(5.3 .13)$. Since the commutators

$$
\begin{aligned}
{\left[\mathcal{B}, \Delta^{2}\right] } & =\text { operator of order } 1+4-1=4 ; \\
{[\mathcal{B}, \Delta] } & =\text { operator of order } 1+2-1=2,
\end{aligned}
$$

we see via the regularity (5.13.15) for $\phi$ and (5.13.17) for $\phi_{t t}$ that the righthand side term $k$ in $(5.13 .23 \mathrm{a})$ satisfies

$$
k \equiv\left[\mathcal{B}, \Delta^{2}\right] \phi+\rho[\mathcal{B}, \Delta] \phi_{t t} \in C\left([0, T] ; L_{2}(\Omega)\right),
$$

recalling $\mathcal{D}(\mathcal{A}) \subset H^{4}(\Omega)$ and $\mathcal{D}\left(\mathcal{A}^{\frac{1}{2}}\right) \subset H^{2}(\Omega)$. Similarly, via (5.13.15), (5.13.16), (5.13.24), as well as by using trace theory, we see that the boundary term $g$ in $(5.13 .23 \mathrm{c})$ satisfies

$$
\begin{aligned}
g & =-\left.[\mathcal{B}, \Delta] \phi\right|_{\Gamma} \in C\left([0, T] ; H^{\frac{3}{2}}(\Gamma)\right) \\
g_{t} & =-\left.[\mathcal{B}, \Delta] \phi_{t}\right|_{\Gamma} \in C\left([0, T] ; H^{\frac{1}{2}}(\Gamma)\right) .
\end{aligned}
$$

Thus, by $(5.13 .23),(5.13 .25),(5.13 .26),(5.13 .27)$, we see that the $z$-problem becomes:

$$
\left\{\begin{array}{l}
z_{t t}-\rho \Delta z_{t t}+\Delta^{2} z=k \in C\left([0, T] ; L_{2}(\Omega)\right) \\
\left.z\right|_{\Sigma} \equiv 0 \\
\left.\Delta z\right|_{\Sigma}=g \in C\left([0, T] ; H^{\frac{3}{2}}(\Gamma)\right) \cap C^{1}\left([0, T] ; H^{\frac{1}{2}}(\Gamma)\right)
\end{array}\right.
$$

with a-priori interior regularity given by (5.13.19)-(5.13.21). We now return to the basic identity (5.10.2). Because of the a-priori interior regularity (5.13.19), (5.13.20), for $\left\{z, z_{t}\right\}$ and that of $k$ in (5.13.28a), the right-hand side (R.H.S.) of identity (5.10.2) (with $\{\phi, f\}$ there replaced by $\{z, k\}$ now) is well defined. Thus, the left-hand side of identity (5.10.2) is well defined. Taking the vector field $h$ such that $\left.h\right|_{\Gamma}=\nu=$ outward unit normal vector on $\Gamma$, we have that

$$
\begin{gathered}
\text { on } \Gamma: \quad h \cdot \nabla(\Delta z)=\nabla(\Delta z) \cdot \nu=\frac{\partial \Delta z}{\partial \nu} ; \quad h \cdot \nabla z_{t}=\frac{\partial \phi_{t}}{\partial \nu} ; \\
\left(\Delta z_{t}\right)^{2} h \cdot \nu=g_{t}^{2} ; \quad\left|\frac{\partial z_{t}}{\partial \nu}\right|=\left|\nabla z_{t}\right|^{2} \text { by (5.13.28b); }
\end{gathered}
$$




$$
\begin{aligned}
\nabla(\Delta z) & =\frac{\partial(\Delta z)}{\partial \nu} \nu+\frac{\partial(\Delta z)}{\partial \nu} \tau, \quad \tau=\text { tangential unit vector on } \Gamma \\
& =\frac{\partial(\Delta z)}{\partial \nu} \nu+\frac{\partial g}{\partial \tau} \tau \\
|\nabla(\Delta z)|^{2} & =\left(\frac{\partial(\Delta z)}{\partial \nu}\right)^{2}+\left(\frac{\partial g}{\partial \tau}\right)^{2}
\end{aligned}
$$

Thus, the left-hand side (L.H.S.) of identity (5.10.2) can be rewritten, in the new variable $z$ as

$$
\begin{aligned}
\text { L.H.S. of (5.10.2) } & =\frac{1}{2} \int_{\Sigma}\left(\frac{\partial(\Delta z)}{\partial \nu}\right)^{2} d \Sigma+\frac{1}{2} \int_{\Sigma}\left(\frac{\partial z_{t}}{\partial \nu}\right)^{2} d \Sigma \\
& +\frac{\rho}{2} \int_{\Sigma} g_{t}^{2} d \Sigma-\frac{1}{2} \int_{\Sigma}\left(\frac{\partial g}{\partial \tau}\right)^{2} d \Sigma \\
& =\text { well defined by R.H.S. of (5.10.2), }
\end{aligned}
$$

since the last two integral terms on the L.H.S. of (5.10.2) vanish due to the B.C. (5.13.28b). The two boundary terms containing $g$ in (5.13.33) are well defined by the regularity of $g$ in $(5.13 .28 \mathrm{c})$, while the boundary term containing $\frac{\partial z_{t}}{\partial \nu}$ is well defined by $(5.13 .20)$ and trace theory. We conclude that the remaining boundary term in $(5.13 .33)$ containing $\frac{\partial(\Delta z)}{\partial \nu}$ is well defined, i.e., $\frac{\partial(\Delta z)}{\partial \nu} \in L_{2}(\Sigma)$, and thus (5.13.22) is established, as desired. The proof of Theorem 5.13.1 is complete.

Remark 5.13.1. In Theorem 5.3.1, the required interior regularity $\left\{\phi, \phi_{t}\right\}$ $\in C\left([0, T] ; H^{3}(\Omega) \times H^{2}(\Omega)\right)$-needed to guarantee that the right-hand side of identity (5.10.2) is well defined - is ensured by the assumed regularity of the data $\left\{\phi_{0}, \phi_{1}, f\right\}$ as in (5.10.1), in particular $f \in L_{1}\left(0, T ; \mathcal{D}\left(\mathcal{A}^{\frac{1}{2}}\right)\right)$, whereby then the (positive) left-hand side of identity (5.10.2) establishes that $\frac{\partial(\Delta \phi)}{\partial \nu} \in L_{2}(\Sigma)$. By contrast, in the z-problem (5.13.28), the righthand side $k$ is only in $C\left([0, T] ; L_{2}(\Omega)\right)$. However, the required regularity $\left\{z, z_{t}\right\} \in C\left([0, T] ; H^{3}(\Omega) \cap H^{2}(\Omega)\right)$ for the right-hand side of identity (5.10.2) is guaranteed by the a-priori regularity $(5.13 .19),(5.13 .20)$, which is a consequence of the regularity (5.13.15), (5.13.16) of $\left\{\phi, \phi_{t}\right\}$ via the change of variable $z=\mathcal{B} \phi$ in (5.13.18). Thus, for the $z$-problem (5.13.28), $k$ is only required to have the regularity that makes the term $\int_{Q} k h \cdot \nabla(\Delta z) d Q$ on the right-hand side of (5.10.2) well defined, i.e., say $k \in L_{1}\left(0, T ; L_{2}(\Omega)\right)$, and we still obtain $\frac{\partial(\Delta z)}{\partial \nu} \in L_{2}(\Sigma)$. The above contrast between the $\phi$-problem in Theorem 5.3.1 and the $z$-problem in (5.13.28) did not occur in the case of the wave equation of [L-T.3, Section 3], while instead is typical for the other illustrating examples: Euler-Bernoulli equation, Schrödinger equations, etc., see [T.1], [L-T.8, Chapter 10]. 


\subsubsection{Completion of the Proof of theorem 5.8.1}

Space regularity. To show space regularity

$$
\begin{gathered}
\left(L^{*} v\right)(t)=\int_{t}^{T} B^{*} e^{A^{*}(\tau-t)} v(\tau) d \tau \\
\quad: \text { continuous } L_{2}\left(0, T ; \mathcal{D}\left(A^{r}\right) \equiv \mathcal{D}\left(\mathcal{A}^{\frac{1}{2}+\frac{r}{4}}\right) \times \mathcal{D}\left(\mathcal{A}^{\frac{1}{2}+\frac{r}{4}}\right)\right) \\
\quad \rightarrow L_{2}\left(0, T ; H^{r}(\Gamma)\right), 0 \leq r \leq 1
\end{gathered}
$$

we simply invoke [L-T.3], [F-L-T.1, Appendix A], which is permissible by virtue of the regularity (5.13.3) of Theorem 5.13.1.

Time regularity. It suffices to show the case $r=1$, since the case $r=0$ is contained in (5.13.35), or in (5.5.6), and then interpolate. Thus, differentiating (5.13.34) in $t$ for

$$
v \in L_{2}(0, T ; \mathcal{D}(A)) \quad \text { or } A^{*} v \in L_{2}(0, T ; Y),
$$

since $A$ is skew-adjoint on $Y$ (see below (5.4.26)) yields

$$
\begin{aligned}
\frac{d\left(L^{*} v\right)}{d t}(t)= & -B^{*} v(t) \\
& -\int_{t}^{T} B^{*} e^{A^{*}(\tau-t)} A^{*} v(\tau) d \tau \in L_{2}\left(0, T ; L_{2}(\Gamma)\right),
\end{aligned}
$$

as desired, by (5.13.36) and $B^{*} A^{*-1} \in \mathcal{L}(Y ; U)$ in (1.5a), $Y$ and $U$ as in (5.4.22). Then (5.13.37) shows

$$
L^{*}: \text { continuous } L_{2}(0, T ; \mathcal{D}(A)) \rightarrow H^{1}\left(0, T ; L_{2}(\Gamma)\right),
$$

as required. The proof of Theorem 5.8.1 is complete except for noticing that uniformity in $s$ is obtained by a change of variable on formula (5.13.34), as usual.

\section{REFERENCES}

[A.1] P. M. Anselone, Collectively Compact Operator Approximation Theory, Prentice Hall, Englewood Cliffs, 1971.

[B-DaP-D-M.1] A. Bensoussan, G. Da Prato, M. Delfour, and S. Mitter, Representation and Control of Infinite Dimensional Systems, II, Birkhäuser, 1992.

[C-L.1] S. Chang and I. Lasiecka, Riccati equations for non-symmetric and nondissipative hyperbolic systems with $L_{2}$-boundary controls, J. Math. Anal. Appl. 116 (1986), 378-414.

[D-L-T.1] G. Da Prato, I. Lasiecka, and R. Triggiani, A direct study of Riccati equations arising in boundary control problems for hyperbolic equations, J. Differential Equations, 64 (1986), 26-47.

[F-L-T] Algebraic Riccati equations with non-smoothing observation arising in hyperbolic and Euler-Bernoulli boundary control problems, Ann. Mat. Pura Appl. (4), 153 (1988), 307-382.

[K.1] H. O. Kreiss, Initial boundary value problems for hyperbolic systems, Comm. Pure Appl. Math. 13 (1970), 277-298. 
[L-L-T.1] I. Lasiecka, J. L. Lions, and R. Triggiani, Non homogeneous boundary value problems for second order hyperbolic operators, J. Math. Pures Appl. 65 (1986), 149-192.

[L-T.1] I. Lasiecka and R. Triggiani, A cosine operator approach to modeling $L_{2}\left(0, T ; L_{2}(\Omega)\right)$ boundary input hyperbolic equations, Appl. Math. Optim. 7 (1981), 35-83.

[L-T.2] I. Lasiecka and R. Triggiani, Regularity of hyperbolic equations under $L_{2}\left(0, T ; L_{2}(\Gamma)\right)$-Dirichlet boundary terms, Appl. Math. Optim. 10 (1983), $275-286$.

[L-T.3] I. Lasiecka and R. Triggiani, Riccati equations for hyperbolic partial differential equations with $L_{2}(\Sigma)$-Dirichlet boundary terms, SIAM J. Control Optim. 24 (1986), 884-926.

[L-T.4] I. Lasiecka and R. Triggiani, Regularity theory for a class of nonhomogeneous Euler-Bernoulli equations: A cosine operator approach, Boll. Un. Mat. Ital. 7, 3-B (1989), 199-228. (Also, invited paper for special volume. Topics in Mathematical Analysis volume dedicated to A. L. Cauchy; (T. M. Rassias ed.), World Scientific, 1989, 623-657.)

[L-T.5] I. Lasiecka and R. Triggiani, Regularity, exact controllability and uniform stabilization of Kirchoff plates with boundary controls only in $\left.\Delta w\right|_{\Sigma}, J$. Differential Equations, 93 (1991), 62-101. Preliminary version in "Semigroup and evolution equations," Lecture Notes in Pure and Applied Mathematics, \#135, Marcel Dekker, New York, 267-295.

[L-T.6] I. Lasiecka and R. Triggiani, Differential and Algebraic Riccati Equations With Applications to Boundary/Point Control Problems: Continuous Theory and Approximation Theory, Lecture Notes in Information and Control, \#164, Springer-Verlag, New York, 1991, 160 pp.

[L-T.7] I. Lasiecka and R. Triggiani, Riccati Differential Equations with unbounded coefficients and non-smoothing terminal condition. The case of analytic semigroups, SIAM J. Math. Anal. 23 (1992), 449-481.

[L-T.8] I. Lasiecka and R. Triggiani, Control Theory for Partial Differential Equations: Continuous and Approximation Theories, I, Encyclopedia of Mathematics and its Applications, Cambridge Univ. Press, to appear in 1997.

[L-M.1] J. L. Lions and J. Magenes, Non-homogeneous Boundary Value Problems and Applications, \#1, Springer-Verlag, Berlin-Heidelberg-New York, 1972.

[Rau.1] J. Rauch, $L_{2}$ is a continuable initial condition for Kreiss' mixed problems, Comm. Pure Appl. Math. 25 (1972), 263-285.

[Ru.1] D. L. Russell, Quadratic performance criteria in boundary control of linear symmetric hyperbolic systems, SIAM J. Control, 11 (1973), 475-509.

[T.1] R. Triggiani, Higher level regularity of Euler-Bernoulli equations and applications to abstract differential Riccati equations, Proceedings of the International Conference on Applied Analysis in the Aegean Archipelagos, July 4-9, 1996, Karlovassi, Samos, Greece, Lecture Notes in Pure and Applied Mathematics, Marcel Dekker, New York, to appear.

\section{Department of Applied Mathematics}

UNIVERSITY OF VIRGINIA

THORNTON HALL

Charlottesville, VA 22903, USA

E-mail address: rt7u@virginia.edu 


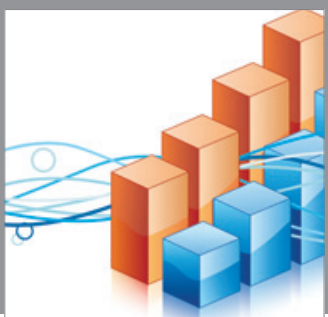

Advances in

Operations Research

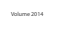

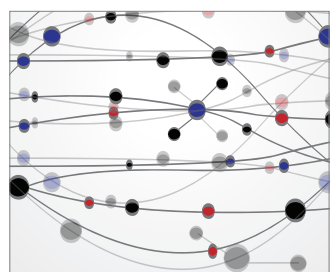

\section{The Scientific} World Journal
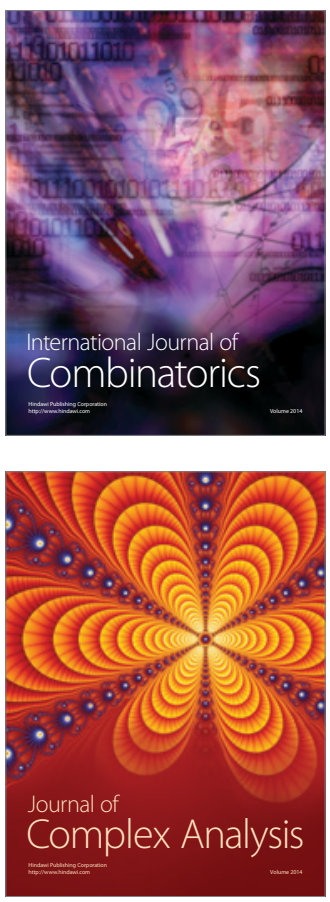

International Journal of

Mathematics and

Mathematical

Sciences
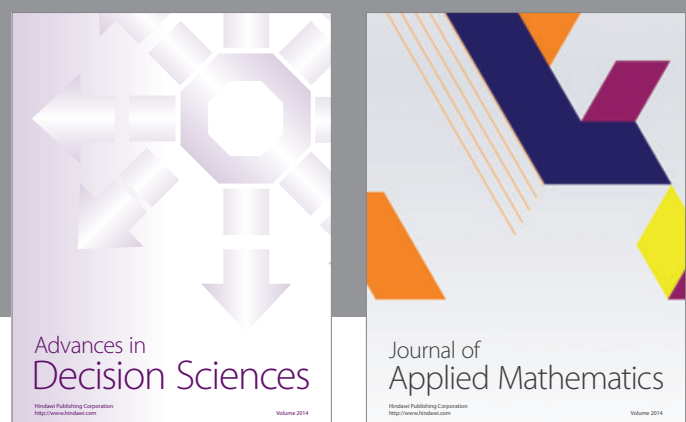

Journal of

Applied Mathematics
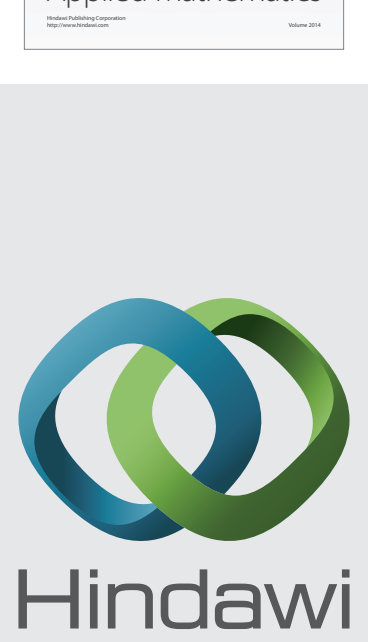

Submit your manuscripts at http://www.hindawi.com
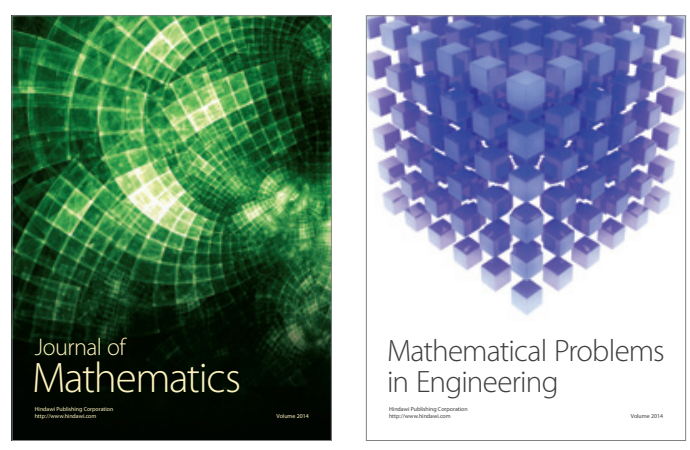

Mathematical Problems in Engineering
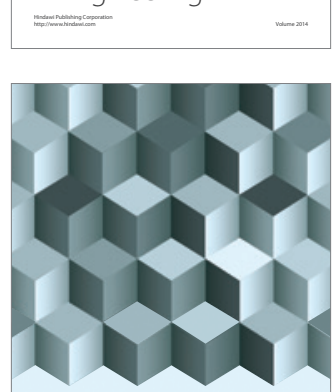

Journal of

Function Spaces
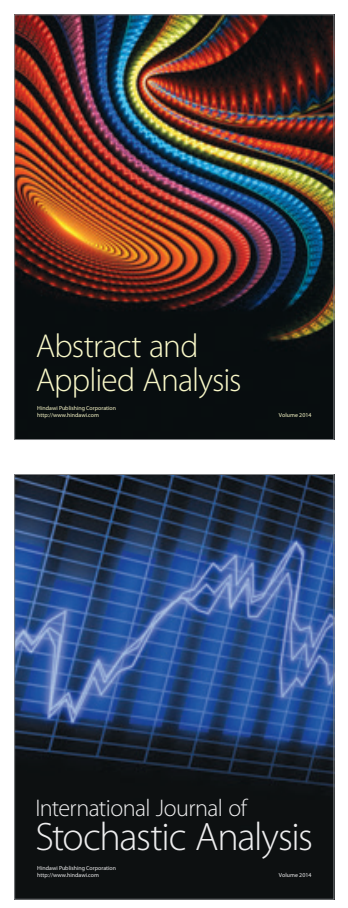

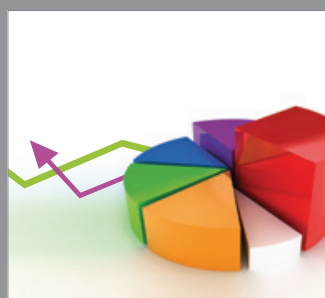

ournal of

Probability and Statistics

Promensencen
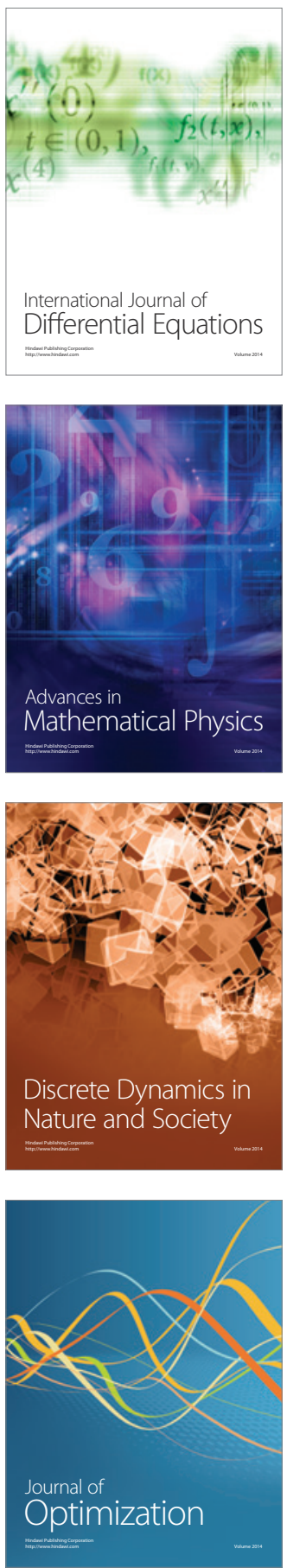